(C) 2000 International Press

Adv. Theor. Math. Phys. 4 (2000) 397-485

\title{
String Partition Functions and Infinite Products
}

Toshiya Kawai and Kōta Yoshioka

\author{
Research Institute for Mathematical Sciences \\ Kyoto University, Kyoto 606-8502, Japan \\ toshiya@kurims.kyoto-u.ac.jp
}

Department of Mathematics, Faculty of Science

Kobe University, Kobe 657-8501, Japan

yoshioka@math.kobe-u.ac.jp

\begin{abstract}
We continue to explore the conjectural expressions of the Gromov-Witten potentials for a class of elliptically and $K 3$ fibered Calabi-Yau 3-folds in the limit where the base $\mathbb{P}^{1}$ of the $K 3$ fibration becomes infinitely large. At least in this limit we argue that the string partition function (= the exponential generating function of the Gromov-Witten potentials) can be expressed as an infinite product in which the Kähler moduli and the string coupling are treated somewhat on an equal footing. Technically speaking, we use the exponential lifting of a weight zero Jacobi form to reach the infinite product as in the celebrated work of
\end{abstract}

e-print archive: http://xxx.lanl.gov/hep-th/0002168 
Borcherds. However, the relevant Jacobi form is associated with a lattice of Lorentzian signature. A major part of this work is devoted to an attempt to interpret the infinite product or more precisely the Jacobi form in terms of the bound states of $D 2$ - and $D 0$-branes using a vortex description and its suitable generalization.

\section{Introduction}

The Gromov-Witten invariants and their potentials have been vigorously investigated in recent years mainly due to their mathematical soundness. See $[6-8,69,73]$ for their fundamental properties. However the Gromov-Witten potentials emerge somewhat indirectly in the conventional physical approaches. Indeed, for Calabi-Yau 3-folds, it is believed [10] that they should appear in the "topological limits" of the naturally defined closed topological A string amplitudes the explicit evaluations of which are prohibitively difficult in general.

In the tests of heterotic/type IIA string duality conjectures, it was desirable to develop the one-loop calculation scheme on the heterotic string side to extract the objects which might correspond to the Gromov-Witten potentials on the type IIA string side. In the pioneering work of Harvey and Moore [49] this task was taken up and certain integrals involving indefinite theta functions were explicitly evaluated on the heterotic string side extending the calculation in [25]. In the course of the calculations they curiously pointed out the relevance of Borcherds' work [12] on holomorphic infinite products. The HarveyMoore method has revealed the presence of a new interesting subject on the theta correspondence and has an advantage when discussing automorphic properties. However several steps were necessary [49] in order to extract the candidate of the genus zero Gromov-Witten potential from the evaluated integral. Recently, the method was extended [78] to cover the Gromov-Witten potentials in higher genera for a particular model using the result of [13] which was itself the extension of the calculations in [49]. In this case also it was necessary to take the limit of a relatively complicated expression to obtain the candidates of the Gromov-Witten potentials. 
Another approach to investigate some features of the Gromov-Witten potentials of Calabi-Yau 3-folds has been advocated by Gopakumar and Vafa [43] using an $M$-theory interpretation and there have been some related works $[53,67]$.

If the Gromov-Witten potentials are of our sole concern, are there any possibilities in which we might directly reach their expressions in all genera? The previous work [65] as well as the present one attempt, albeit in a conjectural and limited sense, to answer this question in the affirmative for a class of elliptically and $K 3$ fibered Calabi-Yau 3 -folds in the limit where the base $\mathbb{P}^{1}$ of the $K 3$ fibration becomes infinitely large. In [65] we tried to interpret the genus $g$ Gromov-Witten potential in terms of the lifting of a Jacobi form of weight $2 g-2$ so that it can be expressed in terms of the "polylogarithm" $\mathrm{Li}_{3-2 g}(\xi)$. There the cases of genus zero and one were discussed in detail while the higher genus cases were briefly speculated upon in the concluding section. The present work further pursues this line of interpretation. Our basic strategy is simple: rather than dealing with the GromovWitten potentials individually we consider the string partition function

$$
\mathcal{Z}=\exp \left(\sum_{g=0}^{\infty} x^{2 g-2} F_{g}\right),
$$

where $F_{g}$ is the genus $g$ Gromov-Witten potential of the fibered CalabiYau 3-fold $Y$ and $x$ is the string coupling parameter. We argue that, in the pertinent limit, $\mathcal{Z}$ can be constructed by the exponential lifting [12] of a weight zero Jacobi form associated with a lattice of Lorentzian signature. Indeed this construction solves the problem at one blow : $F_{g}$ can be expressed as the lifting of a weight $2 g-2$ (quasi) Jacobi form, thus making the statement in [65] precise.

More intriguingly and perhaps more significantly, the construction indicates that $\mathcal{Z}$ can be put (at least in the limit we consider) into an infinite product which resembles the Weyl-Kac-Borcherds denominator. As in [12] the most subtle point in this story is to determine the "Weyl vector" which, we find, should be interpreted as the constant map contributions of the genus zero and one Gromov-Witten potentials. However we have already discussed this technically involved problem in [65] via a felicitous use of elliptic polylogarithms [9]. In fact, one of the motivations for [65] and the present work was a desire to better understand the relation between the Gromov-Witten potentials of the fibered 
Calabi-Yau 3-folds and the original lifting approach of Borcherds [12].

In our construction and the resulting infinite product representation, it turns out to be natural to view $\mathcal{Z}$ as a function (or possibly a section of the appropriate vacuum line bundle) on the "extended moduli space" whose tangent space is some domain of $H^{2}(Y, \mathbb{C}) \oplus H^{0}(Y, \mathbb{C})$. The extended moduli space unifies the complexified Kähler moduli and the string coupling constant and it is natural from the philosophy of "brane democracy". It is also an appropriate setting for the homological mirror conjecture [68]. Thus we should like to have an interpretation of our proposal in terms of the bound states of $D 2$-branes and $D 0$-branes. (In type IIA string theory on Calabi-Yau 3-folds, $D 6$-, $D 4$-branes are electro-magnetic duals of $D 0$-, $D 2$-branes.) In this paper we will make some preliminary (and admittedly modest) efforts toward justification of such an interpretation. In particular, we argue that the bound states of a single $D 2$-brane and $D 0$-branes are described by abelian vortices and their suitable generalizations. We use this interpretation to understand some of the key expressions. In fact, we are able to give a relatively detailed and precise description when the $D 2-D 0$ bound system is in a fixed $K 3$ surface. In such a case, we also point out that the bound state problem of a $D 2$ brane and $D 0$-branes is closely related to vertex operators and their two-point correlation functions.

Presumably the benefit of the lifting procedure employed in this work resides in the very possibility that we may link together, in a rather explicit way, the string perturbative theory of the GromovWitten invariants (which is certainly not brane-democratic but relatively well-understood) and the inherently non-perturbative viewpoint of $D 2$-D0-branes about which we have yet to learn more.

The organization of this paper is as follows. In $\S 2$, we review the fundamental properties of the Gromov-Witten potentials for CalabiYau 3-folds. In $\S 3$ we first recall the general definitions and properties of Jacobi forms as well as those of the Hecke operators. Then we consider the lifting procedure for a class of weight zero Jacobi forms associated with certain Lorentzian lattices and discuss its relation to infinite products. In $\S 4$ we give the main conjecture about the string partition functions of the fibered Calabi-Yau 3-folds. In $\S 5$ we attempt to interpret the proposed expression of the string partition function in terms of the bound states of $D 0$ - and $D 2$-branes. As mentioned above, 
we devote most of this section to the case where the bound system of a single $D 2$-brane and collections of $D 0$-branes is in a $K 3$ surface. Technically the results in [108] turn out to be useful. In $\S 6$ we discuss the relevance of vertex operators and their two-point functions to the $D 2-D 0$ bound state problem. As a simple application of our proposal, we study in $\S 7$ the behavior of the string partition function near the conifold point and relate it to the $S U(\infty)$ Chern-Simons theory on $S^{3}$ thus reproducing the earlier obtained results [41-43, 58,94,104]. In $\S 8$ we raise some directions for further investigations. Several definitions of the functions used in this work and their necessary properties are summarized in Appendix A while Appendix B discusses a conjectural formula of the elliptic genera of the higher order Kummer varieties introduced in [4].

While pursuing the subject of this paper, a paper [62] appeared in which the authors discuss some relevance of the relative Hilbert schemes in conjunction with the proposal of [43]. In our approach the relative Hilbert schemes appear naturally in the $D 2-D 0$-brane bound state interpretation.

Part of this work was presented at the 1998 Kinosaki Symposium on Algebraic Geometry and thenceforth repeated on several occasions. We are grateful to Max-Planck-Institut für Mathematik in Bonn for hospitality. T.K thanks the organizers of the workshop of Activity "Automorphic Products" during which he benefited from conversations with R. Borcherds, R. Dijkgraaf, V.A. Gritsenko, L. Göttsche, S. Kondo, V.V. Nikulin, K. Saito and K. Yoshikawa. We also thank M.-H. Saito for discussions.

\section{Notation.}

$\mathbf{e}[x]=\exp (2 \pi \sqrt{-1} x)$.

$\mathbb{Z}_{+}$: the set of positive integers.

$\mathbb{Z}_{-}$: the set of negative integers.

$\mathbb{N}$ : the set of non-negative integers.

$\mathbb{H}_{g}$ : the Siegel upper space of degree $g$. 


\section{The Gromov-Witten potentials of Calabi-Yau 3-folds}

The Gromov-Witten invariants have been extensively studied in recent years. For the fundamental properties established so far we refer to $[6-8,69,73]$. In this section we review the relevant materials in the cases of Calabi-Yau 3-folds for later convenience.

\subsection{The Gromov-Witten invariants}

Let $Y$ be a smooth Calabi-Yau 3-fold, i.e. a smooth 3-dimensional projective variety over $\mathbb{C}$ with $c_{1}(Y)=0$ and $h^{1,0}(Y)=h^{2,0}(Y)=0$ where $h^{p, q}(Y)=\operatorname{dim} H^{q}\left(Y, \Omega_{Y}^{p}\right)$. Hence $\operatorname{Pic}(Y) \cong H^{2}(Y, \mathbb{Z})$ and $\chi(Y)=$ $2\left(h^{1,1}(Y)-h^{1,2}(Y)\right)$. We assume that $H^{2}(Y, \mathbb{Z})$ is torsion-free. Suppose that $\omega_{1}, \ldots, \omega_{l}$ generate $H^{2}(Y, \mathbb{Z})$ where $l=h^{1,1}(Y)$. Let $D_{1}, \ldots, D_{l}$ be divisors such that $\omega_{i}=c_{1}\left(\mathcal{O}_{Y}\left(D_{i}\right)\right)$ for $i=1, \ldots, l$. Let $\iota_{i}: D_{i} \hookrightarrow Y$ be the inclusions. Then $\omega_{i} \cap[Y]=\left(\iota_{i}\right)_{*}\left[D_{i}\right]$ where [\#] stands for the fundamental homology class of \#. We assume that $D_{1}, \ldots, D_{l}$ are nef so that $\omega_{i} \cap \iota_{*}[C] \geq 0,(i=1, \ldots, l)$ for any algebraic curve $C \subset Y$ with the inclusion $\iota: C \hookrightarrow Y$.

Let $\overline{\mathcal{M}}_{g, n}(Y, \beta)$ be the moduli stack of stable maps where $g, n \geq$ 0 and $\beta \in H_{2}(Y, \mathbb{Z})$. An element of $\overline{\mathcal{M}}_{g, n}(Y, \beta)$ is represented by $\left(\Sigma_{g}, p_{1}, \ldots, p_{n}, \varphi\right)$. Here $\Sigma_{g}$ is a connected curve of arithmetic genus $g=\operatorname{dim} H^{1}\left(\Sigma_{g}, \mathcal{O}_{\Sigma_{g}}\right)$ whose only possible singularities are ordinary double points while $p_{1}, \ldots, p_{n}$ are distinct nonsingular points on $\Sigma_{g}$. The last entry is a morphism $\varphi: \Sigma_{g} \rightarrow Y$ such that $\left\{\mu \in\right.$ Aut $\Sigma_{g} \mid$ $\left.\varphi \circ \mu=\varphi, \mu\left(p_{i}\right)=p_{i}\right\}$ is finite and $\varphi_{*}\left[\Sigma_{g}\right]=\beta$.

Let

$$
\pi: \overline{\mathcal{C}}_{g, n}(Y, \beta) \rightarrow \overline{\mathcal{M}}_{g, n}(Y, \beta)
$$

be the universal curve over $\overline{\mathcal{M}}_{g, n}(Y, \beta)$. We have $\overline{\mathcal{C}}_{g, n}(Y, \beta)=$ $\overline{\mathcal{M}}_{g, n+1}(Y, \beta)$. Set

$$
\begin{aligned}
& f: \quad \overline{\mathcal{C}}_{g, n}(Y, \beta) \quad \longrightarrow \quad Y \\
& \left(\Sigma_{g}, p_{1}, \ldots, p_{n+1}, \varphi\right) \longmapsto \varphi\left(p_{n+1}\right) .
\end{aligned}
$$

The virtual dimension of $\overline{\mathcal{M}}_{g, n}(Y, \beta)$ is often smaller than the actual dimension of $\overline{\mathcal{M}}_{g, n}(Y, \beta)$. The virtual fundamental class $\left[\overline{\mathcal{M}}_{g, n}(Y, \beta)\right]^{\text {vir }}$ 
can be constructed so that its dimension coincides with the virtual dimension of $\overline{\mathcal{M}}_{g, n}(Y, \beta)[6-8,73]$. This construction uses the obstruction sheaf $R^{1} \pi_{*} f^{*} T_{Y}$, where $T_{Y}$ is the tangent sheaf of $Y$, and is given by

$$
\left[\overline{\mathcal{M}}_{g, n}(Y, \beta)\right]^{\mathrm{vir}}=e\left(R^{1} \pi_{*} f^{*} T_{Y}\right) \cap\left[\overline{\mathcal{M}}_{g, n}(Y, \beta)\right],
$$

if $R^{1} \pi_{*} f^{*} T_{Y}$ is locally-free. Here $e()$ represents the Euler class. Intuitively, $e\left(R^{1} \pi_{*} f^{*} T_{Y}\right)$ represents the contribution from the anti-ghost zero modes.

For a Calabi-Yau 3-fold $Y$, the virtual dimension of $\overline{\mathcal{M}}_{g, n}(Y, \beta)$ is equal to $n$. Using the evaluation maps

$$
\begin{aligned}
& \mathrm{ev}_{i}: \quad \overline{\mathcal{M}}_{g, n}(Y, \beta) \quad \longrightarrow \quad Y \\
& \left(\Sigma_{g}, p_{1}, \ldots, p_{n}, \varphi\right) \longmapsto \varphi\left(p_{i}\right),
\end{aligned}
$$

the Gromov-Witten invariants are introduced by

$$
\left\langle\omega_{i_{1}} \cdots \omega_{i_{n}}\right\rangle_{g, \beta}=\left(\operatorname{ev}_{1}^{*}\left(\omega_{i_{1}}\right) \cup \cdots \cup \operatorname{ev}_{n}^{*}\left(\omega_{i_{n}}\right)\right) \cap\left[\overline{\mathcal{M}}_{g, n}(Y, \beta)\right]^{\mathrm{vir}} .
$$

We extend the Gromov-Witten invariants by $\mathbb{C}$-linearity:

$$
\left\langle t_{i_{1}} \omega_{i_{1}} \cdots t_{i_{n}} \omega_{i_{n}}\right\rangle_{g, \beta}=t_{i_{1}} \cdots t_{i_{n}}\left\langle\omega_{i_{1}} \cdots \omega_{i_{n}}\right\rangle_{g, \beta}
$$

for $t_{i_{1}}, \ldots, t_{i_{n}} \in \mathbb{C}$.

\subsection{The Gromov-Witten potentials and their known general properties}

If we write $\omega=\sum_{i} t_{i} \omega_{i} \in \mathcal{K}_{\mathbb{C}} \subset H^{2}(Y, \mathbb{C})$ where $\mathcal{K}_{\mathbb{C}}$ is the complexified Kähler cone, the Gromov-Witten invariants can be compactly organized into the Gromov-Witten potentials:

$$
F_{g}=\sum_{\beta \in H_{2}(Y, \mathbb{Z})}\left\langle e^{\omega}\right\rangle_{g, \beta}
$$

since

$$
F_{g}=\sum_{\beta} \sum_{n \geq 0} \frac{1}{n !}\left\langle\omega^{n}\right\rangle_{g, \beta}=\sum_{\beta} \sum_{n \geq 0} \sum_{i_{1}, \ldots, i_{n}} \frac{t_{i_{1}} \cdots t_{i_{n}}}{n !}\left\langle\omega_{i_{1}} \cdots \omega_{i_{n}}\right\rangle_{g, \beta} .
$$


By the fundamental property of topological sigma models or the Divisor Axiom [69], it follows that

$$
\left\langle\omega^{n}\right\rangle_{g, \beta}=(\omega \cap \beta)^{n}\langle 1\rangle_{g, \beta}
$$

for $\beta \neq 0$. Hence we have

$$
F_{g}=\left\langle e^{\omega}\right\rangle_{g, 0}+\sum_{\beta \neq 0}\langle 1\rangle_{g, \beta} e^{\omega \cap \beta}
$$

Let $C_{0} \subset Y$ be a rigid smooth rational curve $C_{0} \subset Y$ with normal bundle $N=\mathcal{O}_{C_{0}}(-1) \oplus \mathcal{O}_{C_{0}}(-1)$. Fix a positive integer $h$. Let $p: \overline{\mathcal{C}}_{g, 0}\left(C_{0}, h\left[C_{0}\right]\right) \rightarrow \overline{\mathcal{M}}_{g, 0}\left(C_{0}, h\left[C_{0}\right]\right)$ be the universal curve and $\mu:$ $\overline{\mathcal{C}}_{g, 0}\left(C_{0}, h\left[C_{0}\right]\right) \rightarrow C_{0}$ the universal evaluation map. It was conjectured in [44] and proved ${ }^{1}$ in [31] that the multiple covering effect of $C_{0}$ can be summarized by

$$
e\left(R^{1} p_{*} \mu^{*} N\right) \cap\left[\overline{\mathcal{M}}_{g, 0}\left(C_{0}, h\left[C_{0}\right]\right)\right]^{\mathrm{vir}}=m_{g} h^{2 g-3},
$$

where $m_{g}$ are the rational numbers defined through

$$
\left(y^{-1 / 2}-y^{1 / 2}\right)^{-2}=-\sum_{g=0}^{\infty} x^{2 g-2} m_{g}, \quad y=\exp (\sqrt{-1} x) .
$$

Explicitly we have $m_{0}=1, m_{1}=\frac{1}{12}$ and in general

$$
m_{g}=\frac{(-1)^{g-1}(2 g-1) B_{2 g}}{(2 g) !} .
$$

For $g>1$, it follows that

$$
m_{g}=\frac{(-1)^{g-1} \chi_{g, 0}}{(2 g-3) !}
$$

where we use the formula of the orbifold Euler characteristic of the moduli space of genus $g(>1)$ curves with $n$ punctures [48]:

$$
\chi_{g, n}=(-1)^{n}\left(\begin{array}{c}
2 g-3+n \\
n
\end{array}\right) \frac{B_{2 g}}{2 g(2 g-2)} .
$$

\footnotetext{
${ }^{1}$ See also $[2,10,76]$.
} 
Therefore the multiple coverings of rational curves should contribute to the second term on the right hand side of (2.10) in the form

$$
\sum_{\beta^{\prime} \neq 0} \sum_{h>0}\langle 1\rangle_{0, \beta^{\prime}} m_{g} h^{2 g-3} e^{\omega \cap\left(h \beta^{\prime}\right)}=\sum_{\beta^{\prime} \neq 0}\langle 1\rangle_{0, \beta^{\prime}} m_{g} \operatorname{Li}_{3-2 g}\left(e^{\omega \cap \beta^{\prime}}\right),
$$

where the "polylogarithm" function $\operatorname{Li}_{3-2 g}(\xi)$ is defined in Appendix A.

The evaluation of the constant map contribution $\left\langle e^{\omega}\right\rangle_{g, 0}$ has been explicitly performed in the literature $[10,37,70]$. We briefly recall this. By the isomorphism

$$
\overline{\mathcal{M}}_{g, n}(Y, 0) \cong \overline{\mathcal{M}}_{g, n} \times Y,
$$

we have $\pi=\tilde{\pi} \times$ id with the universal curve $\tilde{\pi}: \overline{\mathcal{C}}_{g, n} \rightarrow \overline{\mathcal{M}}_{g, n}$. Set $\mathbb{E}=$ $\tilde{\pi}_{*} \omega_{\overline{\mathcal{C}}_{g, n} / \overline{\mathcal{M}}_{g, n}}$ where $\omega_{\overline{\mathcal{C}}_{g, n} / \overline{\mathcal{M}}_{g, n}}$ is the relative dualizing sheaf [88]. Thus $\mathbb{E}^{*}=R^{1} \tilde{\pi}_{*} \mathcal{O}_{\overline{\mathcal{C}}_{g, n}}$ by duality and it follows that $R^{1} \pi_{*} f^{*} T_{Y} \cong \mathbb{E}^{*} \otimes T_{Y}$. Consequently, we have

$$
\left[\overline{\mathcal{M}}_{g, n}(Y, 0)\right]^{\mathrm{vir}}=c_{g \operatorname{dim}(Y)}\left(\mathbb{E}^{*} \otimes T_{Y}\right) \cap\left[\overline{\mathcal{M}}_{g, n}(Y, 0)\right],
$$

where we used $\operatorname{rk}(\mathbb{E})=g$. Then the evaluation of $\left\langle e^{\omega}\right\rangle_{g, 0}$ reduces to the Hodge integrals, i.e. the integrals over $\overline{\mathcal{M}}_{g, n}$ of cup products of the Chern classes $\lambda_{i}:=c_{i}(\mathbb{E})$.

Set $\mathbb{S}:=\mathbb{N}^{l} \backslash\{0\}$. We regard $\mathbb{S}$ as a poset by the partial ordering: $d^{\prime} \leq d\left(d, d^{\prime} \in \mathbb{S}\right)$ iff $d_{i}^{\prime} \mid d_{i}\left({ }^{\forall} i\right)$. Let us introduce new variables $q_{1}=e^{t_{1}}, \ldots, q_{l}=e^{t_{l}}$. If $d=\left(d_{1}, \ldots, d_{l}\right) \in \mathbb{S}$ we write $q^{d}$ for $q_{1}^{d_{1}} \cdots q_{l}^{d_{l}}$. We also introduce

$$
\begin{aligned}
\kappa_{i j k} & =D_{i} \cdot D_{j} \cdot D_{k}=\left(\omega_{i} \cup \omega_{j} \cup \omega_{k}\right) \cap[Y], \\
\rho_{i} & =c_{2}(Y) \cdot D_{i}=c_{2}(Y) \cap\left(\iota_{i}\right)_{*}\left[D_{i}\right]=\left(c_{2}(Y) \cup \omega_{i}\right) \cap[Y] .
\end{aligned}
$$

Then combining the above results the Gromov-Witten potentials were found to have the following expressions:

$$
\begin{aligned}
& F_{0}=\frac{1}{3 !} \sum_{i, j, k} \kappa_{i j k} t_{i} t_{j} t_{k}-\frac{\chi(Y)}{2} \zeta(3)+\sum_{d \in \mathbb{S}} N_{0}(d) m_{0} \operatorname{Li}_{3}\left(q^{d}\right) \\
& F_{1}=-\lambda_{1} \cap\left[\overline{\mathcal{M}}_{1,1}\right] \cdot \sum_{i} \rho_{i} t_{i}+\sum_{d \in \mathbb{S}}\left[N_{0}(d) m_{1}+\sum_{\substack{d^{\prime} \in \mathbb{S} \\
d^{\prime} \leq d}} N_{1}\left(d^{\prime}\right)\right] \operatorname{Li}_{1}\left(q^{d}\right),
\end{aligned}
$$


and

$$
F_{g}=(-1)^{g} \lambda_{g-1}^{3} \cap\left[\overline{\mathcal{M}}_{g, 0}\right] \cdot \frac{\chi(Y)}{2}+\sum_{d \in \mathbb{S}} N_{0}(d) m_{g} \operatorname{Li}_{3-2 g}\left(q^{d}\right)+\cdots,
$$

for $g>1$. The coefficients $N_{0}(d)$ and $N_{1}(d)$ count the primitive numbers of rational and elliptic curves.

Remark 2.23. In $F_{0}$ we have inserted the term $-\frac{\chi(Y)}{2} \zeta(3)$ by hand. This term seems to lack a satisfactory explanation in the pure context of the Gromov-Witten theory but, as well-known, its existence has been supported from other approaches. Since $\mathrm{Li}_{3}(\xi)$ and $\mathrm{Li}_{1}(\xi)$ are multivalued functions with non-trivial monodromy groups (see Appendix A and [65] for a summary) we neglected the terms that can be cancelled by monodromy transformations in the expressions of $F_{0}$ and $F_{1}$. Recall that $\zeta(3)$ is irrational so that $-\frac{\chi(Y)}{2} \zeta(3)$ cannot be cancelled by a monodromy transformation.

A basic result due to Mumford [88] is:

$$
\lambda_{1} \cap\left[\overline{\mathcal{M}}_{1,1}\right]=\frac{1}{24} .
$$

Another important result is:

$$
\lambda_{g-1}^{3} \cap\left[\overline{\mathcal{M}}_{g, 0}\right]=(-1)^{g-1} m_{g} \zeta(3-2 g), \quad(g>1) .
$$

This equation (rewritten in an equivalent form) was conjectured in [30] and recently proved in [31]. See also [78] [43] for physical justification.

Thus we have seen that $F_{g}$ contains the constant term proportional to $\zeta(3-2 g)$ and is related to the function $\operatorname{Li}_{3-2 g}(\xi)$. (For $F_{1}$ we have not considered the term proportional to $\zeta(1)$ since $\zeta(1)$ is divergent. However, as we will see later, its formal presence may be preferred from some aesthetic viewpoint.) In the following we will see that these features of $F_{g}$ are indeed realized in our conjectural expressions.

\section{$3 \quad$ Jacobi forms and their liftings}

The purpose of this section is to collect together some fundamental materials of Jacobi forms whose properties are indispensable for our 
construction. In the simplest case a systematic study of Jacobi forms was initiated in [27]. A straightforward extension of [27] leads to the idea of Jacobi forms associated with positive definite lattices. However, for our present purpose, it is necessary to consider Jacobi forms associated with lattices of Lorentzian signature. We note that such possibilities have already been considered in [40] in the context of the Donaldson invariants for 4-manifolds with $b_{2}^{+}=1$.

\subsection{Jacobi forms}

Let $(\Pi,\langle\rangle$,$) be an even integral lattice, i.e. a free \mathbb{Z}$-module $\Pi$ of finite rank endowed with a symmetric non-degenerate bilinear form $\langle\rangle:, \Pi \times \Pi \rightarrow \mathbb{Z}$ satisfying $\langle\boldsymbol{\lambda}, \boldsymbol{\lambda}\rangle \in 2 \mathbb{Z}$ for all $\boldsymbol{\lambda} \in \Pi$. Note that we allow $\Pi$ to be indefinite. As is customary, we write $\Pi$ instead of $(\Pi,\langle\rangle$, when the bilinear form is known from the context. We also write $\Pi(r)$ for $(\Pi, r\langle\rangle$,$) where r \in \mathbb{Q}$. The bilinear form $\langle$,$\rangle determines the$ canonical embedding $\Pi \subset \Pi^{*}=\operatorname{Hom}_{\mathbb{Z}}(\Pi, \mathbb{Z})$. By extending $\langle$,$\rangle via$ $\mathbb{Q}$-linearity we can regard $\Pi^{*}$ as a rational lattice. We also identify $\Pi_{\mathbb{C}}$ with $\Pi_{\mathbb{C}}^{*}$ by extending $\langle$,$\rangle via \mathbb{C}$-linearity. Given a nonzero rational number $r$, let $\langle r\rangle$ denote the rank 1 lattice $(\mathbb{Z} e,\langle\rangle$,$) with the generator$ $e$ satisfying $\langle e, e\rangle=r$.

We assume that $\Pi^{*}$ is such that any element of it is either positive, zero or negative.

Definition 3.1. A triplet $(\ell, n, \gamma) \in \mathbb{Z} \times \mathbb{Z} \times \Pi^{*}$ is said to be positive if either of the following three cases holds:
(i) $\ell>0$,
(ii) $\ell=0, n>0$
(iii) $\ell=n=0, \gamma>0$.

We write $(\ell, n, \gamma)>0$ if $(\ell, n, \gamma)$ is positive.

Definition 3.2. A Jacobi form of weight $k \in \mathbb{Z}$ associated with $\Pi=$ $(\Pi,\langle\rangle$,$) is a meromorphic function \Phi_{k}: \mathbb{H}_{1} \times \Pi_{\mathbb{C}} \rightarrow \mathbb{C}$ satisfying

1. For any $\left(\begin{array}{ll}a & b \\ c & d\end{array}\right) \in S L_{2}(\mathbb{Z})$,

$$
\Phi_{k}\left(\frac{a \tau+b}{c \tau+d}, \frac{\boldsymbol{z}}{c \tau+d}\right)=(c \tau+d)^{k} \mathbf{e}\left[\frac{c\langle\boldsymbol{z}, \boldsymbol{z}\rangle}{2(c \tau+d)}\right] \Phi_{k}(\tau, \boldsymbol{z}) .
$$


2. For any $\boldsymbol{\lambda}, \boldsymbol{\mu} \in \Pi$,

$$
\Phi_{k}(\tau, \boldsymbol{z}+\boldsymbol{\lambda} \tau+\boldsymbol{\mu})=\mathbf{e}\left[-\left(\frac{\langle\boldsymbol{\lambda}, \boldsymbol{\lambda}\rangle}{2} \tau+\langle\boldsymbol{\lambda}, \boldsymbol{z}\rangle\right)\right] \Phi_{k}(\tau, \boldsymbol{z}) .
$$

3. $\Phi_{k}$ can be Fourier-expanded in some appropriate region of $\mathbb{H}_{1} \times \Pi_{\mathbb{C}}$ as

$$
\Phi_{k}(\tau, \boldsymbol{z})=\sum_{\substack{n \geq-n_{0} \\ \boldsymbol{\gamma} \in \Pi^{*}}} D(n, \boldsymbol{\gamma}) q^{n} \boldsymbol{\zeta}^{\boldsymbol{\gamma}}
$$

where $n_{0}$ is some non-negative integer and we have introduced the notation $q=\mathbf{e}[\tau]$ and $\boldsymbol{\zeta}^{\boldsymbol{\gamma}}=\mathbf{e}[\langle\boldsymbol{\gamma}, \boldsymbol{z}\rangle]$.

Remark 3.6. Since $\Phi_{k}(\tau,-\boldsymbol{z})=(-1)^{k} \Phi_{k}(\tau, \boldsymbol{z})$, we have $D(n,-\gamma)=$ $(-1)^{k} D(n, \gamma)$.

Definition 3.7. Suppose that $(\Pi,\langle\rangle$,$) is positive definite. Then \Phi_{k}$ in Definition 3.2 is said to be nearly holomorphic if $n_{0}>0$ while it is said to be weak if $n_{0}=0$.

Let $\mathfrak{g}$ be a simple Lie algebra of rank $s$ with a fixed Cartan subalgebra $\mathfrak{h}$ and $W(\mathfrak{g})$ the Weyl group of $\mathfrak{g}$. We identify $\mathfrak{h}$ with $\mathfrak{h}^{*}$ using the Killing form $($,$) . We extend ($,$) by \mathbb{C}$-linearity. We normalize the highest root $\theta$ as $(\theta, \theta)=2$. Let $Q^{\vee}=\left(Q^{\vee},(),\right)$ be the coroot lattice of $\mathfrak{g}$. Then $Q^{\vee}$ is a positive definite even integral lattice of rank $s$ and $P=\left(Q^{\vee}\right)^{*}$ is the weight lattice of $\mathfrak{g}$. With this data we used in [65] the notion of Weyl-invariant Jacobi forms following [100]:

Definition 3.8. A Weyl-invariant Jacobi form $\phi_{k, m}$ of weight $k$ and index $m$ is a Jacobi form of weight $k$ associated with the lattice $Q^{\vee}(m)$ in the sense of Definition 3.2 such that it is invariant under the action of $W(\mathfrak{g})$ on $Q^{\vee}(m)_{\mathbb{C}}$.

We note that a weak Jacobi form of even weight in the sense of [27] is a weak Weyl-invariant Jacobi form of $A_{1}$.

Let

$$
E_{2 k}(\tau)=1-\frac{4 k}{B_{2 k}} \sum_{n=1}^{\infty} \sigma_{2 k-1}(n) q^{n}, \quad(k \geq 1),
$$

denote the normalized Eisenstein series of weight $2 k$ where $\sigma_{k}(n)=$ $\sum_{d \mid n} d^{k}$ 
Definition 3.10. A meromorphic function on $\mathbb{H}_{1} \times \Pi_{\mathbb{C}}$ is called a quasi Jacobi form of weight $k$ associated with $\Pi$ if it is expressed for some integer $k_{0}$ as $\sum_{k^{\prime}=k_{0}}^{k} p_{k-k^{\prime}}\left(E_{2}, E_{4}, E_{6}\right) \Phi_{k^{\prime}}$ where $\Phi_{k^{\prime}}$ is a Jacobi form of weight $k^{\prime}$ associated with $\Pi$ and $p_{k-k^{\prime}}\left(E_{2}, E_{4}, E_{6}\right) \in \mathbb{C}\left[E_{2}, E_{4}, E_{6}\right]$ is a quasi modular form [61] of weight $k-k^{\prime}$.

\subsection{Hecke operators and liftings}

In this section we assume that $\Phi_{k}$ is a quasi Jacobi form of weight $k$ associated with an even integral lattice $\Pi$ having Fourier expansion (3.5).

Definition 3.11. For $\ell=1,2, \ldots$ the action of the Hecke operator $V_{\ell}$ on $\Phi_{k}$ is defined, as in [27], by

$$
\left.\Phi_{k}\right|_{V_{\ell}}(\tau, \boldsymbol{z}):=\ell^{k-1} \sum_{\substack{a d=\ell \\ a>0}} \sum_{b=0}^{d-1} d^{-k} \Phi_{k}\left(\frac{a \tau+b}{d}, a z\right) .
$$

The following relation has already been used in [65]:

\section{Lemma 3.13.}

$$
\left.\sum_{\ell=1}^{\infty} p^{\ell} \Phi_{k}\right|_{V_{\ell}}(\tau, \boldsymbol{z})=\sum_{\substack{\ell, n, \gamma \\ \ell>0}} D(\ell n, \gamma) \operatorname{Li}_{1-k}\left(p^{\ell} q^{n} \boldsymbol{\zeta}^{\gamma}\right)
$$

Proof. From the definition (3.12) the left hand side is equal to

$$
\sum_{\ell=1}^{\infty} p^{\ell} \ell^{k-1} \sum_{\substack{a d=\ell \\ a>0}} \sum_{b=0}^{d-1} d^{-k} \sum_{n, \boldsymbol{\gamma}} D(n, \gamma) \mathbf{e}[b n / d] q^{n a / d}\left(\boldsymbol{\zeta}^{\gamma}\right)^{a}
$$

By performing the sum over $b$ we obtain

$$
\begin{gathered}
\sum_{\ell=1}^{\infty} p^{\ell} \ell^{k-1} \sum_{\substack{a d=\ell \\
a>0}} d^{-k+1} \sum_{n, \boldsymbol{\gamma}} D(n d, \gamma) q^{n a}\left(\boldsymbol{\zeta}^{\gamma}\right)^{a} \\
=\sum_{d=1}^{\infty} \sum_{n, \boldsymbol{\gamma}} D(n d, \gamma) \sum_{a=1}^{\infty} a^{k-1}\left(p^{d} q^{n} \boldsymbol{\zeta}^{\gamma}\right)^{a}
\end{gathered}
$$


However, the last expression is equal to the right hand side of (3.14).

This lemma urges us to introduce:

Definition 3.17. The action of the Hecke operator $V_{0}$ on $\Phi_{k}$ is defined by

$$
\left.\Phi_{k}\right|_{V_{0}}(\tau, \boldsymbol{z}):=\frac{D(0, \mathbf{0})}{2} \zeta(1-k)+\sum_{(0, n, \boldsymbol{\gamma})>0} D(0, \boldsymbol{\gamma}) \operatorname{Li}_{1-k}\left(q^{n} \boldsymbol{\zeta}^{\boldsymbol{\gamma}}\right)
$$

Combining (3.14) and (3.18) we find that

\section{Lemma 3.19.}

$$
\left.\sum_{\ell=0}^{\infty} p^{\ell} \Phi_{k}\right|_{V_{\ell}}(\tau, \boldsymbol{z})=\frac{D(0, \mathbf{0})}{2} \zeta(1-k)+\sum_{(\ell, n, \boldsymbol{\gamma})>0} D(\ell n, \gamma) \mathrm{Li}_{1-k}\left(p^{\ell} q^{n} \boldsymbol{\zeta}^{\gamma}\right)
$$

Remark 3.21. Since $\zeta(1)$ diverges, the definition (3.18) and hence (3.19) are meaningless for $k=0$ as they stand. Nevertheless the case $k=0$ is the most important one in the next subsection. To treat this case adequately one would have to make an analytical continuation in $k$ and regularize the divergence properly. However, in the following we will adopt a simple-minded approach keeping $\zeta(1)$ as the divergent sum $\sum_{h>0} \frac{1}{h}$ in the intermediate process of calculations and discard the diverging $\zeta(1)$ in the end. Hopefully this will make the manipulations below transparent although they are admittedly formal.

\subsection{Lorentzian lattices and Jacobi forms of weight zero}

So far we have been quite general. In the following we will choose a specific Lorentzian lattice $\Pi$ and an associated Jacobi form $\Phi_{0}$ of weight zero.

Fix a simple Lie algebra $\mathfrak{g}$ of rank $s$ (with the convention mentioned before) and an associated nearly holomorphic Weyl-invariant Jacobi 
form of weight -2 and index $m$ denoted henceforth as $\phi_{-2, m}$. We will focus on the even Lorentzian lattice of signature $(s, 1)$ :

$$
\Pi=Q^{\vee}(m) \oplus\langle-2\rangle .
$$

The reason why we select this lattice will become clear in the next section. We parametrize the elements of $\Pi_{\mathbb{C}}$ as

$$
\Pi_{\mathbb{C}} \ni \boldsymbol{z}=z \oplus \nu e,
$$

where $z \in Q^{\vee}(m)_{\mathbb{C}}$ and $\nu \in \mathbb{C}$ with $e$ being the generator of $\langle-2\rangle$.

Since we have $\Pi^{*}=P\left(\frac{1}{m}\right) \oplus\left\langle-\frac{1}{2}\right\rangle$, we write

$$
\Pi^{*} \ni \gamma=\gamma \oplus j e^{*}
$$

where $\gamma \in P\left(\frac{1}{m}\right)$ and $j \in \mathbb{Z}$ with $e^{*}$ being the generator of $\left\langle-\frac{1}{2}\right\rangle$. Then we say $\gamma>0$ if either of the following possibilities holds

$$
\text { (i) } \gamma>0 \text {, (ii) } \gamma=0 \text { and } j>0 \text {. }
$$

We write $(\ell, n, \gamma, j)>0$ when $(\ell, n, \gamma)>0$. We also write $(\ell, n, \gamma)>0$ when $(\ell, n, \gamma, j)>0$ but the restriction on $j$ is removed.

Consider

$$
E(\tau, \nu):=-\sqrt{-1} \frac{\vartheta_{1}(\tau, \nu)}{\eta(\tau)^{3}}, \quad(\tau, \nu) \in \mathbb{H}_{1} \times \mathbb{C},
$$

where

$$
\vartheta_{1}(\tau, \nu)=\sqrt{-1}\left(y^{-1 / 2}-y^{1 / 2}\right) q^{1 / 8} \prod_{n=1}^{\infty}\left(1-q^{n}\right)\left(1-q^{n} y\right)\left(1-q^{n} y^{-1}\right)
$$

is the odd Jacobi theta function and

$$
\eta(\tau)=q^{\frac{1}{24}} \prod_{n=1}^{\infty}\left(1-q^{n}\right)
$$

is the Dedekind $\eta$ function. Obviously,

$$
E(\tau, \nu)=\left(y^{-1 / 2}-y^{1 / 2}\right) \prod_{n=1}^{\infty} \frac{\left(1-q^{n} y\right)\left(1-q^{n} y^{-1}\right)}{\left(1-q^{n}\right)^{2}} .
$$


Moreover, this can be expressed in terms of the Eisenstein series:

$$
E(\tau, \nu)=-\sqrt{-1} x \exp \left(\sum_{k=1}^{\infty} \frac{(-1)^{k} B_{2 k}}{2 k(2 k) !} x^{2 k} E_{2 k}(\tau)\right),
$$

where

$$
y=\mathbf{e}[\nu] \text { and } \quad x=2 \pi \nu .
$$

The function $E(\tau, \nu)$ is essentially the prime form on the elliptic curve with modulus $\tau$. It is easy to see that $E(\tau, \nu)^{2}$ is a weak Jacobi form of weight -2 and index 1 in the sense of [27] and it actually coincides with $\widetilde{\phi}_{-2,1}(\tau, \nu)$ in [27], which is one of the two generators of the ring of weak Jacobi forms with even weights.

We then define

$$
\Phi_{0}(\tau, \boldsymbol{z})=\Phi_{0}(\tau, z, \nu):=\frac{\phi_{-2, m}(\tau, z)}{E(\tau, \nu)^{2}},
$$

which is apparently a Jacobi form of weight zero associated with $\Pi$. Since we have

$$
\begin{aligned}
\frac{1}{E(\tau, \nu)^{2}} & =\frac{1}{\left(y^{-1 / 2}-y^{1 / 2}\right)^{2}} \prod_{n=1}^{\infty} \frac{\left(1-q^{n}\right)^{4}}{\left(1-q^{n} y\right)^{2}\left(1-q^{n} y^{-1}\right)^{2}} \\
& =-\frac{1}{x^{2}} \exp \left(\sum_{k=1}^{\infty} \frac{(-1)^{k-1} B_{2 k}}{k(2 k) !} x^{2 k} E_{2 k}(\tau)\right)
\end{aligned}
$$

we can asymptotically expand $\Phi_{0}(\tau, z, \nu)$ as

$$
\Phi_{0}(\tau, z, \nu)=-\sum_{g=0}^{\infty} x^{2 g-2} \varphi_{2 g-2, m}(\tau, z),
$$

where $\varphi_{2 g-2, m}$ is a quasi Jacobi form obtained from $\phi_{-2, m}$ by multiplying a weight $2 g$ quasi modular form, i.e. an element of weight $2 g$ in $\mathbb{Q}\left[E_{2}, E_{4}, E_{6}\right]$. Apparently we have

$$
\varphi_{-2, m}(\tau, z)=\phi_{-2, m}(\tau, z) .
$$

We expand $\varphi_{2 g-2, m}$ as

$$
\varphi_{2 g-2, m}(\tau, z)=\sum_{n, \gamma} c_{g}(n, \gamma) q^{n} \zeta^{\gamma},
$$


where $\zeta^{\gamma}=\mathbf{e}[(\gamma, z)]$. Then we have the symmetry property $c_{g}(n, \gamma)=$ $c_{g}(n,-\gamma)$.

The expression $\left(y^{-1 / 2}-y^{1 / 2}\right)^{-2}$ appearing in (3.32) has subtle features and will play an important role later in this paper. It has expansions

$$
\left(y^{-1 / 2}-y^{1 / 2}\right)^{-2}=\sum_{j=1}^{\infty} j y^{ \pm j}, \quad(|y| \lessgtr 1),
$$

exhibiting a wall-crossing behavior. On the other hand, precisely on the wall, we have

$$
\left(y^{-1 / 2}-y^{1 / 2}\right)^{-2}=\frac{1}{2} \sum_{j \in \mathbb{Z}}|j| y^{j}, \quad(|y|=1, y \neq 1) .
$$

In the rest of this section and the next section we will tacitly assume that we are precisely on the wall, hence the expansion (3.37). We may thus regard the expression $\left(y^{-1 / 2}-y^{1 / 2}\right)^{-2}$ as an element of $\frac{1}{2} \mathbb{Z}\left[\left[y, y^{-1}\right]\right]$ by interpreting it as a formal distribution [60]. The reason for assuming (3.37) is that the Fourier expansion

$$
\Phi_{0}(\tau, z, \nu)=\sum_{n, \gamma, j} D(n, \gamma, j) q^{n} \zeta^{\gamma} y^{j}
$$

has the manifest symmetry properties

$$
D(n, \gamma, j)=D(n,-\gamma, j)=D(n, \gamma,-j)
$$

Note that we must have $c_{0}(n, \gamma) \in 2 \mathbb{Z}$ if we demand $D(n, \gamma, j) \in \mathbb{Z}$.

However, when we attempt an interpretation in terms of $D 2-D 0$ bound states in $\S 5$ we shall be mostly off the wall using the expansion (3.36).

Lemma 3.40.

$$
\sum_{j} D(n, \gamma, j) y^{j}=-\sum_{g=0}^{\infty} x^{2 g-2} c_{g}(n, \gamma) .
$$

Proof. This is a direct consequence of (3.33). 
Now we would like to consider the actions of the Hecke operators on $\Phi_{0}$ and $\varphi_{2 g-2, m}$ and compare the results. For simplicity we will use the same notation $V_{\ell}(\ell=0,1,2, \ldots)$ for both $\Phi_{0}$ and $\varphi_{2 g-2, m}$. For $\varphi_{2 g-2, m}$, the Hecke operator $V_{0}$ is defined by using the expansion (3.35). Since we are dealing with (quasi) Jacobi forms of weight zero we should emphasize again what we have cautioned in Remark 3.21.

The following identity is crucial for our purpose:

\section{Lemma 3.42.}

$$
\left.\Phi_{0}\right|_{V_{\ell}}(\tau, z, \nu)=-\left.\sum_{g=0}^{\infty} x^{2 g-2} \varphi_{2 g-2, m}\right|_{V_{\ell}}(\tau, z), \quad(\ell=0,1,2, \ldots)
$$

Proof. If $\ell>0$, we find that

$$
\begin{aligned}
\left.\Phi_{0}\right|_{V_{\ell}}(\tau, z, \nu) & =\ell^{-1} \sum_{\substack{a d=\ell \\
a>0}} \sum_{b=0}^{d-1} \Phi_{0}\left(\frac{a \tau+b}{d}, a z, a \nu\right) \\
& =\ell^{-1} \sum_{\substack{a d=\ell \\
a>0}} \sum_{\substack{-1 \\
b=0}}^{\infty}\left(-\sum_{g=0}^{\infty}(a x)^{2 g-2} \varphi_{2 g-2, m}\left(\frac{a \tau+b}{d}, a z\right)\right) \\
& =-\sum_{g=0}^{\infty} x^{2 g-2} \ell^{2 g-3} \sum_{\substack{a d=\ell \\
a>0}} \sum_{b=0}^{d-1} d^{-(2 g-2)} \varphi_{2 g-2, m}\left(\frac{a \tau+b}{d}, a z\right) \\
& =-\left.\sum_{g=0}^{\infty} x^{2 g-2} \varphi_{2 g-2, m}\right|_{V_{\ell}}(\tau, z) .
\end{aligned}
$$


As for the case $\ell=0$, we have

$$
\begin{aligned}
\left.\Phi_{0}\right|_{V_{0}}(\tau, z, \nu)= & \frac{1}{2} D(0,0,0) \zeta(1)+\sum_{(0, n, \gamma, j)>0} D(0, \gamma, j) \operatorname{Li}_{1}\left(q^{n} \zeta^{\gamma} y^{j}\right) \\
= & \frac{1}{2} D(0,0,0) \zeta(1)+\sum_{(0, n, \gamma, j)>0} D(0, \gamma, j) \sum_{h>0} \frac{\left(q^{n} \zeta^{\gamma} y^{j}\right)^{h}}{h} \\
= & \frac{1}{2} \sum_{h>0} \frac{1}{h} \sum_{j} D(0,0, j) y^{j h} \\
& \quad+\sum_{h>0} \sum_{(0, n, \gamma)>0} \frac{\left(q^{n} \zeta^{\gamma}\right)^{h}}{h} \sum_{j} D(0, \gamma, j) y^{j h}
\end{aligned}
$$

where we used $\zeta(1)=\sum_{h>0} \frac{1}{h}$. Thus Lemma 3.40 shows that

$$
\begin{aligned}
& \left.\Phi_{0}\right|_{V_{0}}(\tau, z, \nu) \\
& =-\frac{1}{2} \sum_{h>0} \frac{1}{h} \sum_{g=0}^{\infty}(h x)^{2 g-2} c_{g}(0,0) \\
& -\sum_{h>0} \sum_{(0, n, \gamma)>0} \frac{\left(q^{n} \zeta^{\gamma}\right)^{h}}{h} \sum_{g=0}^{\infty}(h x)^{2 g-2} c_{g}(0, \gamma) \\
& =-\sum_{g=0}^{\infty} x^{2 g-2}\left(\frac{c_{g}(0,0)}{2} \zeta(3-2 g)+\sum_{(0, n, \gamma)>0} c_{g}(0, \gamma) \operatorname{Li}_{3-2 g}\left(q^{n} \zeta^{\gamma}\right)\right) \\
& =-\left.\sum_{g=0}^{\infty} x^{2 g-2} \varphi_{2 g-2, m}\right|_{V_{0}}(\tau, z) \text {. }
\end{aligned}
$$

This completes the proof of (3.43).

Now we set

$$
\mathcal{F}_{g}:=\left.\sum_{\ell=0}^{\infty} p^{\ell} \varphi_{2 g-2, m}\right|_{V_{\ell}}(\tau, z),
$$

as in [65]. We will see in the next section that $\mathcal{F}_{g}$ is an important piece of the Gromov-Witten potential $F_{g}$ for certain elliptically and $K 3$ fibered Calabi-Yau 3-folds. 
Remark 3.48. Note however that even for $g \geq 2, \mathcal{F}_{g}$ is not exactly an automorphic form on the type IV domain but what might be called a quasi automorphic form since we are using a quasi Jacobi form $\varphi_{2 g-2, m}$ for the lifting. The situation is reminiscent of that in [23] where the enumerative problem associated with the Riemann-Hurwitz theory for elliptic curves was discussed and the connection to quasi modular forms [61] were explained. At hindsight the encounter with quasi automorphic forms is inevitable and should be interpreted as the remnant of the holomorphic anomaly studied in [10]. It also partially explains why some extra work is needed when one uses the Harvey-Moore method [49] to extract the Gromov-Witten potentials: in the Harvey-Moore method the automorphic properties are always preserved while what we are after are not precisely automorphic forms. Although not simply related to the Gromov-Witten potentials, still it might be possible to preserve the automorphic property by replacing $\varphi_{2 g-2, m}$ by a genuine Jacobi form $\phi_{2 g-2, m}$ as expected in [65]. At least this was already done in the genus one case.

Lemma 3.19 then tells us that

\section{Proposition 3.49.}

$$
\mathcal{F}_{g}=\frac{c_{g}(0,0)}{2} \zeta(3-2 g)+\sum_{(\ell, n, \gamma)>0} c_{g}(\ell n, \gamma) \operatorname{Li}_{3-2 g}\left(p^{\ell} q^{n} \zeta^{\gamma}\right) .
$$

The following infinite product is an essential ingredient when we discuss the string partition function in the next section:

\section{Proposition 3.51.}

$$
\exp \left(\sum_{g=0}^{\infty} x^{2 g-2} \mathcal{F}_{g}\right)=e^{-\frac{D(0,0,0)}{2} \zeta(1)} \prod_{(\ell, n, \gamma, j)>0}\left(1-p^{\ell} q^{n} \zeta^{\gamma} y^{j}\right)^{D(\ell n, \gamma, j)}
$$

Proof. We see that

$$
\exp \left(\sum_{g=0}^{\infty} x^{2 g-2} \mathcal{F}_{g}\right)=\exp \left(-\left.\sum_{\ell=0}^{\infty} p^{\ell} \Phi_{0}\right|_{V_{\ell}}(\tau, z, \nu)\right),
$$

by Lemma 3.42. Then (3.52) follows from Lemma 3.19. 


\section{String partition function}

By utilizing the results obtained in the above we formulate in this section the conjectures on the Gromov-Witten potentials and the string partition function for Calabi-Yau 3-folds endowed with specific fibration structures.

\subsection{Fibered Calabi-Yau 3-folds}

Let $Y$ denote a Calabi-Yau 3-fold as in $\S 2$. In this section we use the notations introduced there. We now list up what we will assume on $Y$. First, we assume that there exist a $K 3$ fibration $\pi_{1}: Y \rightarrow W_{1}$ as well as an elliptic fibration $\pi_{2}: Y \rightarrow W_{2}$. The two fibrations are assumed to be compatible. This implies that a generic fiber of $\pi_{1}$ is an elliptic $K 3$ surface. We mostly assume that $\pi_{2}: Y \rightarrow W_{2}$ has a section. Next we assume that all the singular fibers of $\pi_{1}: Y \rightarrow W_{1}$ are irreducible. Then $W_{1} \cong \mathbb{P}^{1}$ and $W_{2} \cong \mathbb{F}_{a}(a=0,1, \ldots, 12)$ where $\mathbb{F}_{a}=\mathbb{P}_{\mathbb{P}^{1}}\left(\mathcal{O}_{\mathbb{P}^{1}} \oplus \mathcal{O}_{\mathbb{P}^{1}}(a)\right)$ is a Hirzebruch surface. See for instance, [54]. (In general, the allowed possibilities of the base of an elliptic Calabi-Yau 3 -fold with a section are del Pezzo, Enriques, Hirzebruch or blown-up Hirzebruch surfaces [83].)

Furthermore the Picard lattice of a generic fiber of $\pi_{1}: Y \rightarrow W_{1}$, which is necessarily an elliptic $K 3$, is assumed to coincide with $H \oplus$ $Q^{\vee}(-m)$ where $H$ is the hyperbolic plane, i.e. the even unimodular indefinite lattice of rank 2 with intersection matrix $\left(\begin{array}{ll}0 & 1 \\ 1 & 0\end{array}\right), m$ is some positive integer and $Q^{\vee}$ is the coroot lattice of some simple Lie algebra $\mathfrak{g}$ of rank $s=l-3$.

With these assumptions we express the complexified Kähler parameters as in [65]:

$$
\begin{aligned}
t_{1} & =\log u-\log q-\frac{a}{2}(\log p-\log q), \\
t_{2} & =\log p-\log q \\
t_{3} & =\log q-\left(\gamma_{0}, \log \zeta\right) \\
t_{i+3} & =\left(\Lambda_{i}, \log \zeta\right), \quad(i=1, \ldots, s),
\end{aligned}
$$

where $\Lambda_{i}(i=1, \ldots, s)$ are the fundamental weights of $\mathfrak{g}$ and $\gamma_{0}$ is some 
positive weight. This parametrization is such that $\omega_{1}$ is the pullback via $\pi_{1}$ of the fundamental cohomology class of $W_{1}$. We have

$$
\rho_{1}=24, \quad \rho_{2}=24+12 a, \quad \rho_{3}=92 .
$$

The parametrization (4.1) should allow us to fix a particular "fundamental chamber" in which we work in the following.

\subsection{The main conjectures}

As in [65] we assume that there exists a nearly holomorphic Jacobi form of weight -2 and index $m$ associated with $Y$ in the form

$$
\phi_{-2, m}(\tau, z)=\frac{\Psi_{10, m}(\tau, z)}{\eta(\tau)^{24}},
$$

where $\Psi_{10, m}(\tau, z)$ is a weak Weyl-invariant (with respect to $\mathfrak{g}$ ) Jacobi form of weight 10 and index $m$ satisfying $\Psi_{10, m}(\tau, 0)=-2 E_{4}(\tau) E_{6}(\tau)$. Sadly, we are aware of neither a general algorithm to determine the precise form of $\Psi_{10, m}(\tau, z)$ from the geometric information of $Y$ nor whether there are additional conditions on $Y$ for $\phi_{-2, m}$ to exist. However at least we must have

$$
c_{0}(-1, \gamma)=0, \quad \text { for } \gamma \neq 0, \quad c_{0}(-1,0)=-2, \quad c_{0}(0,0)=-\chi(Y),
$$

for the following conjectures to make sense. We substitute (4.3) in the definition (3.31) of $\Phi_{0}$. We assume that the coefficients $D(n, \gamma, j)$ are integers so that $c_{0}(n, \gamma)$ are even integers.

Now we can state our conjectures on the Gromov-Witten potentials:

Conjecture 4.5. The Gromov-Witten potentials behave as

$$
\begin{aligned}
& F_{0}=F_{0}^{(0)}+\mathcal{F}_{0}+O\left(q_{1}\right), \\
& F_{1}=F_{1}^{(0)}+\mathcal{F}_{1}+O\left(q_{1}\right), \\
& F_{g}=\mathcal{F}_{g}+O\left(q_{1}\right), \quad \text { for } g>1,
\end{aligned}
$$

where $\mathcal{F}_{g}$ are given by (3.50) and

$$
\begin{aligned}
& F_{0}^{(0)}=\frac{1}{3 !} \sum_{i, j, k} \kappa_{i j k} t_{i} t_{j} t_{k}, \\
& F_{1}^{(0)}=-\lambda_{1} \cap\left[\overline{\mathcal{M}}_{1,1}\right] \cdot \sum_{i} \rho_{i} t_{i}=-\frac{1}{24} \sum_{i} \rho_{i} t_{i} .
\end{aligned}
$$


In the fundamental chamber, we gave in [65] conjectural formulas of $F_{0}^{(0)}$ and $F_{1}^{(0)}$ expressed in terms of the data of $\phi_{-2, m}$. This was achieved by employing the elliptic polylogarithm of Beilinson and Levin [9] which is the holomorphic version of that of Zagier [110]. The result reads as follows:

Conjecture 4.8. In the fundamental chamber we have

$$
\begin{aligned}
F_{0}^{(0)} & =\log u\left\{\log p \log q-\frac{m}{2}(\log \zeta, \log \zeta)\right\} \\
& +\left(\frac{m}{2}-\frac{\mathcal{I}_{2}}{24 s}\right) \log p \cdot(\log \zeta, \log \zeta) \\
& +\frac{1}{3}(\log q)^{3}-\frac{\mathcal{I}_{2}}{24 s} \log q \cdot(\log \zeta, \log \zeta)+\frac{1}{12} \sum_{\gamma>0} c_{0}(0, \gamma)(\gamma, \log \zeta)^{3}
\end{aligned}
$$

$a n d^{2}$

$$
F_{1}^{(0)}=-\frac{1}{24}(24 \log u+24 \log p+44 \log q)+\frac{1}{24} \sum_{\gamma>0} c_{0}(0, \gamma)(\gamma, \log \zeta)
$$

where $\log \zeta=2 \pi \sqrt{-1} z$ and $\mathcal{I}_{2}=\sum_{\gamma>0} c_{0}(0, \gamma)(\gamma, \gamma)$

As shown in [65] these formulas are such that if we replace $\operatorname{Li}_{r}(r=1,3)$ by $\mathcal{L i}_{r}(r=1,3)$, we have (at least to the first order in $\left.q_{1}\right)$

$$
F_{g}(u, p, q, \zeta)=F_{g}\left(u^{\prime}, q, p, \zeta\right)
$$

for all $g \geq 0$, where

$$
\log u^{\prime}=\log u-(\log p-\log q) .
$$

For the definition of $\mathcal{L} \mathrm{i}_{r}$ see Appendix A.

Since we have

$$
c_{g}(-1,0)=m_{g} c_{0}(-1,0)
$$

and

$$
c_{g}(0,0)=m_{g} c_{0}(0,0), \quad(g \neq 1), \quad c_{1}(0,0)=m_{1} c_{0}(0,0)-2 c_{0}(-1,0),
$$

\footnotetext{
${ }^{2}$ The normalization of $F_{1}$ differs from that in [65] by $m_{1}=\frac{1}{12}$.
} 
it is easy to check that the conjectured expressions of $F_{g}$ are consistent with the general results reviewed in $\S 2$.

For concrete examples of $Y$ and some corroboration of Conjectures 4.5 and 4.8 for $g=0,1$, see [65] and references therein.

If we translate these conjectures on the Gromov-Witten potentials into the language of the string partition function using Proposition 3.51, we reach the main conjecture of this paper:

Conjecture 4.15. The string partition function behaves as

$$
\mathcal{Z}=\exp \left(x^{-2} F_{0}^{(0)}+F_{1}^{(0)}\right)\left[\prod_{(\ell, n, \gamma, j)>0}\left(1-p^{\ell} q^{n} \zeta^{\gamma} y^{j}\right)^{D(\ell n, \gamma, j)}+O\left(q_{1}\right)\right]
$$

where we neglected $\zeta(1)$ appearing in (3.52).

Eq. (4.16) bears strong resemblance to Borcherds' infinite product formulas $[12]^{3}$. This should be so since we have employed more or less the same kind of lifting procedure. However, the important difference lies in that we used the lifting of a weight zero Jacobi form associated with a Lorentzian lattice. This has entailed a more complicated expression of the "Weyl vector" $x^{-2} F_{0}^{(0)}+F_{1}^{(0)}$ which exhibits chamber dependence as in the case of the ordinary Weyl vector. It should be noted that since $F_{0}^{(0)}$ and $F_{1}^{(0)}$ are respectively homogeneously cubic and linear in $t_{i}$, the homogeneous degree of $x^{-2} F_{0}^{(0)}+F_{1}^{(0)}$ as a function of $x$ and $t_{i}$ is one, lending further support to the interpretation of $x^{-2} F_{0}^{(0)}+F_{1}^{(0)}$ as the "Weyl vector".

\section{An interpretation in terms of $D 2-D 0$ bound states}

In eq. (4.16) we observe that the complexified Kähler moduli and the string coupling $x$ are unified in a rather nice way. In fact, the

\footnotetext{
${ }^{3}$ When making this analogy, it should be born in mind that there is a conventional ambiguity: we could replace $\mathcal{Z}$ by $\mathcal{Z}^{-1}$ in the definition of the string partition function.
} 
geometrical origin of the Lorentzian lattice $\Pi$ we have used may be attributed to the following relation:

$$
H^{2}(Y, \mathbb{Z}) \oplus H^{0}(Y, \mathbb{Z}) \supset H \oplus Q^{\vee}(-m) \oplus\langle 2\rangle=H \oplus \Pi(-1),
$$

where we identified $H^{0}(Y, \mathbb{Z})$ with the lattice $\langle 2\rangle$. The (analytically continued) string coupling $x$ parametrizes $H^{0}(Y, \mathbb{C})$ just as the complexified Kähler moduli parametrize (a cone of) $H^{2}(Y, \mathbb{C})$. Thus it seems natural to view the string partition function as a function (or a section of the appropriate vacuum line bundle) over the extended moduli space whose tangent space is some domain of $H^{2}(Y, \mathbb{C}) \oplus H^{0}(Y, \mathbb{C})$. This fact immediately suggests that there should be an interpretation of the infinite product (4.16) in terms of the bound states of $D 2$ - and $D 0$-branes. In the following we wish to develop some arguments supporting this picture.

Remark 5.2. As mentioned in Introduction, $D 6$ - and $D 4$-branes are duals of $D 0$ - and $D 2$-branes. Thus the above extended moduli space is a half of the usual extended moduli space [103] whose tangent space is contained in $\oplus_{i=0}^{3} H^{2 i}(Y, \mathbb{C})$.

In the conjectured expression (4.16) all the information is encoded in the Jacobi form $\Phi_{0}$ or its Fourier coefficients $D(n, \gamma, j)$. Our basic expectation in the following is that $\Phi_{0}$ should be interpreted as the function counting the bound states of a single $D 2$-brane and $D 0$-branes moving inside the fibers of the $K 3$ fibration.

\subsection{Preliminaries}

\subsubsection{Notations}

For a smooth complex projective variety $V$, we define the Hodge polynomial by

$$
\chi_{t, \tilde{t}}(V):=\sum_{p, q=0}^{\operatorname{dim}(V)}(-1)^{p+q} h^{p, q}(V) t^{p} \tilde{t}^{q},
$$

where $h^{p, q}(V)=\operatorname{dim} H^{q}\left(V, \Omega_{V}^{p}\right)$. We also introduce

$$
\chi_{t}(V):=\chi_{t, 1}(V)
$$


which is essentially the Hirzebruch $\chi_{y}$ genus of $V$. Note that the Euler characteristic of $V$ is given by $\chi(V)=\chi_{1}(V)$.

The $r^{\text {th }}$ symmetric group $\mathfrak{S}_{r}$ naturally acts on $V^{r}=V \times \cdots \times V(r$ times) as permutations on $r$ letters. The quotient is the $r^{\text {th }}$ symmetric product $V^{(r)}:=V^{r} / \mathfrak{S}_{r}$. In general $V^{(r)}$ has orbifold singularities when $\operatorname{dim}(V)>1$ while it is smooth when $\operatorname{dim}(V)=1$. We set $V^{(0)}=\{\mathrm{pt}\}$.

Let $V$ be an even dimensional Calabi-Yau manifold. Then the elliptic genus $\mathcal{E}_{V}(\tau, \nu)$ is a weak Jacobi form of weight 0 and index $\operatorname{dim}(V) / 2[66]$ with the expansion

$$
\mathcal{E}_{V}(\tau, \nu)=y^{-\operatorname{dim}(V) / 2} \chi_{y}(V)+O(q) .
$$

We have the duality relation $\chi_{y^{-1}}(V)=y^{-\operatorname{dim}(V)} \chi_{y}(V)$.

Let $V$ be a complex algebraic variety of dimension $n$ and $E$ a coherent sheaf on $V$. We denote by $\operatorname{Supp}(E)$ the support of $E$. The dimension of $E$, denoted as $\operatorname{dim}(E)$, is defined to be that of $\operatorname{Supp}(E)$ and $E$ is called of pure dimension $m$ if $\operatorname{dim}(F)=m$ for all nontrivial coherent subsheaves $F \subset E$.

In the following, by $H_{k}(V, \mathbb{A})$ we always mean the $k^{\text {th }}$ Borel-Moore homology group [16] with coefficients in a commutative ring $\mathbb{A}$. The fundamental homology class of $V$, which is an element of $H_{2 n}(V, \mathbb{Z})$, is denoted by $[V]$. If $V$ is smooth, the operation $\cap[V]$ gives the Poincaré duality isomorphism: $H^{k}(V, \mathbb{Z}) \cong H_{2 n-k}(V, \mathbb{Z})$. If $V$ is smooth and compact, the Borel-Moore homology coincides with the ordinary one.

If $W \subset V$ is a closed subvariety with the inclusion $\iota: W \hookrightarrow V$, we frequently write $[W]$ instead of $\iota_{*}[W]$.

In this section we usually denote by $X$ a projective $K 3$ surface.

\subsubsection{D-brane charges}

We recall some generalities on $D$-brane charges. It has been argued-[50] that $D$-brane charges in $X$ are associated with Mukai vectors. The Mukai lattice of $X$ is the total integer cohomology group

$$
H^{2 *}(X, \mathbb{Z})=H^{0}(X, \mathbb{Z}) \oplus H^{2}(X, \mathbb{Z}) \oplus H^{4}(X, \mathbb{Z}),
$$


endowed with the symmetric bilinear form

$$
\left\langle v, v^{\prime}\right\rangle_{X}=c_{1} \cdot c_{1}^{\prime}-a b^{\prime}-a^{\prime} b,
$$

for any $v=\left(a, c_{1}, b\right) \in H^{2 *}(X, \mathbb{Z})$ and $v^{\prime}=\left(a^{\prime}, c_{1}^{\prime}, b^{\prime}\right) \in H^{2 *}(X, \mathbb{Z})$. Here the notation $v=\left(a, c_{1}, b\right)$ means $v=a \oplus c_{1} \oplus b$ with $a \in H^{0}(X, \mathbb{Z}), c_{1} \in$ $H^{2}(X, \mathbb{Z})$ and $b \in H^{4}(X, \mathbb{Z})$. We have $H^{2 *}(X, \mathbb{Z}) \cong \mathrm{E}_{8}(-1)^{\oplus 2} \oplus H^{\oplus 4}$ where $E_{8}$ is the positive definite even unimodular lattice of rank 8 .

The Grothendieck group $K_{0}(X)$ is defined to be the quotient of the free abelian group generated by all the coherent sheaves (up to isomorphisms) on $X$ by the subgroup generated by the elements $F-$ $E-G$ for each short exact sequence

$$
0 \rightarrow E \rightarrow F \rightarrow G \rightarrow 0
$$

of coherent sheaves on $X$. In what follows, we shall use the same notation $E$ for both a coherent sheaf on $X$ and its image in $K_{0}(X)$.

Let $v: K_{0}(X) \rightarrow \oplus_{i} H^{2 i}(X, \mathbb{Q})$ be the module homomorphism defined by Mukai vectors [85-87], namely $E \mapsto v(E):=\operatorname{ch}(E) \sqrt{\operatorname{td}(X)}$. Explicitly we have

$$
v(E)=\left(\operatorname{rk}(E), c_{1}(E), \operatorname{rk}(E) \varrho+\frac{1}{2} c_{1}(E)^{2}-c_{2}(E)\right)
$$

where $\varrho \in H^{4}(X, \mathbb{Z})$ is the fundamental cohomology class of $X$ so that $\varrho \cap[X]=1$. Thus actually we have $v\left(K_{0}(X)\right) \subset H^{2 *}(X, \mathbb{Z})$ since $H^{2}(X, \mathbb{Z})$ is even. This definition is such that

$$
\chi(E, F):=\sum_{i=0}^{2}(-1)^{i} \operatorname{dim} \operatorname{Ext}^{i}(E, F)=-\langle v(E), v(F)\rangle_{X}
$$

by the Hirzebruch-Riemann-Roch theorem.

The Mukai lattice has several distinguished isometries. For instance, for an invertible sheaf $L$ on $X$, the map

$$
\begin{aligned}
& v(E) \mapsto \operatorname{ch}(L) v(E) \\
& =v(E)+\left(0, \operatorname{rk}(E) c_{1}(L), c_{1}(E) \cup c_{1}(L)+\frac{\operatorname{rk}(E)}{2} c_{1}(L)^{2}\right),
\end{aligned}
$$


gives an isometry of $H^{2 *}(X, \mathbb{Z})$.

Let $Q: K_{0}(X) \rightarrow \oplus_{i} H_{2 i}(X, \mathbb{Q})$ be defined by

$$
\begin{aligned}
E \mapsto Q(E): & =v(E) \cap[X] \\
& =\left(\operatorname{rk}(E)[X], c_{1}(E) \cap[X], \chi(X, E)-\operatorname{rk}(E)\right) .
\end{aligned}
$$

We call $Q(E)$ the $D$-brane charge of $E$ with its component in $H_{2 i}$ representing the $D 2 i$-brane charge. Since $Q$ is a module homomorphism, it follows that

$$
Q(F)=Q(E)+Q(G),
$$

for each exact sequence (5.8) of coherent sheaves on $X$. This may be interpreted as the charge conservation law when making the D-brane state associated with $F$ out of those associated with $E$ and $G$.

Let $C$ be a curve on $X$ and let $\iota: C \hookrightarrow X$ be the inclusion. If $E$ is a coherent sheaf on $C$, the direct image $\iota_{*} E$ is a torsion sheaf on $X$ obtained by "extending by zero". Suppose that we have the exact sequence (5.8) now for coherent sheaves on $C$. Since $\iota_{*}$ is an exact functor, we have

$$
Q\left(\iota_{*} F\right)=Q\left(\iota_{*} E\right)+Q\left(\iota_{*} G\right) .
$$

This may also be regarded as the charge conservation law for D-brane states without $D 4$-branes. Similar formula holds for a 0 -dimensional subscheme instead of $C$.

The above consideration can be extended almost verbatim to a smooth Calabi-Yau manifold $Y$ of any dimension. We define $Q$ : $K_{0}(Y) \rightarrow \oplus_{i} H_{2 i}(Y, \mathbb{Q})$ by $E \mapsto Q(E):=v(E) \cap[Y]$ where $v(E)=$ $\operatorname{ch}(E) \sqrt{\operatorname{td}(Y)}$.

Remark 5.15. In the above, we have defined $Q$ on the Grothendieck group $K_{0}(Y)$. However, in more general contexts like homological mirror conjecture [68] or Fourier-Mukai transforms, the domain of $Q$ must be (naturally) extended from $K_{0}(Y)$ to the bounded derived category $\mathbf{D}^{b}(Y)$ of coherent sheaves on $Y$.

Let $V$ be a smooth variety of dimension $n$ and $W$ an $m$-dimensional irreducible and reduced subvariety of $V$ with the inclusion map $\iota$ : $W \hookrightarrow V$. Then by using a resolution of singularities $\pi: \tilde{W} \rightarrow W$ and the Grothendieck-Riemann-Roch theorem for singular varieties [34], one can show $[20, \S 5.8-5.9]$ that 


\section{Lemma 5.16.}

$$
\begin{aligned}
\operatorname{ch}_{k}\left(\iota_{*} \mathcal{O}_{W}\right) \cap[V] & =0, \quad \text { for } k<n-m, \\
\operatorname{ch}_{n-m}\left(\iota_{*} \mathcal{O}_{W}\right) \cap[V] & =[W] .
\end{aligned}
$$

Suppose that a coherent sheaf $E$ on $V$ is of pure dimension $m$. Let $\operatorname{Supp}(E)=\cup_{i} S_{i}$ be the support of $E$, where $S_{i}$ are irreducible and reduced. We shall define the multiplicity of $E$ along $S_{i}$. Let $\mathcal{O}_{V, S_{i}}$ be the stalk of $\mathcal{O}_{V}$ at $S_{i}$ and $E_{V, S_{i}}$ the stalk of $E$ at $S_{i}$. Let $I_{S_{i}, S_{i}}$ be the stalk of the ideal sheaf of $S_{i}$. Then $\left(\mathcal{O}_{V, S_{i}}, I_{S_{i}, S_{i}}\right)$ is a local ring with the residue field $\mathcal{O}_{V, S_{i}} / I_{S_{i}, S_{i}} \cong K\left(S_{i}\right)$, where $K\left(S_{i}\right)$ is the function field of $S_{i}$. Since $\left(I_{S_{i}, S_{i}}\right)^{k} E_{V, S_{i}}=0$ for some $k$, there is a filtration

$$
0 \subset F_{i}^{1} \subset F_{i}^{2} \subset \cdots \subset F_{i}^{s_{i}}=E_{V, S_{i}}
$$

such that $F_{i}^{j} / F_{i}^{j-1} \cong K\left(S_{i}\right)$. We define the multiplicity of $E$ along $S_{i}$ by $\operatorname{mult}_{S_{i}}(E):=s_{i}$. Namely, mult $_{S_{i}}(E)$ is the length of $E_{V, S_{i}}$ as an $\mathcal{O}_{V, S_{i}}$-module.

Lemma 5.19.

$$
\begin{aligned}
\operatorname{ch}_{k}(E) & \cap[V]=0, \quad \text { for } k<n-m, \\
\operatorname{ch}_{n-m}(E) \cap[V] & =\sum_{i} \operatorname{mult}_{S_{i}}(E)\left[S_{i}\right] .
\end{aligned}
$$

Proof. See [20, §5.8- 5.9].

Proposition 5.21. Suppose that $Y$ is a (smooth) Calabi-Yau manifold.

(i) Let $\iota: Z \hookrightarrow Y$ be a 0-dimensional subscheme of length d. Then,

$$
Q\left(\iota_{*} \mathcal{O}_{Z}\right)=(0, \ldots, 0, d) .
$$

(ii) Let $E$ be a coherent sheaf of pure dimension 1 on $Y$. If $\operatorname{Supp}(E)=$ $\cup_{i} C_{i}$ with $C_{i}$ being irreducible and reduced, then

$$
Q(E)=\left(0, \ldots, 0, \sum_{i} \operatorname{mult}_{C_{i}}(E)\left[C_{i}\right], \chi(Y, E)\right) .
$$

Proof. (i) is an easy consequence of Lemma 5.19 while (ii) follows from Lemma 5.19, $c_{1}(Y)=0$ and Riemann-Roch. 
Corollary 5.24. Let $\iota: C \hookrightarrow Y$ be an irreducible and reduced curve on $Y$ and $F$ a torsion-free sheaf on $C$. Then,

$$
Q\left(\iota_{*} F\right)=(0, \ldots, 0, \operatorname{rk}(F)[C], \chi(C, F))
$$

Proof. Recall that

$$
\chi(C, F)=\chi\left(Y, \iota_{*} F\right),
$$

since $H^{i}(C, F) \cong H^{i}\left(Y, \iota_{*} F\right)$ for the inclusion $\iota$.

Remark 5.27. If $C$ is smooth, Corollary 5.24 can also be seen (as done in [50] for the case $n=2$ ) by directly using the Grothendieck-RiemannRoch theorem for nonsingular varieties:

$$
\operatorname{ch}\left(\iota_{!} F\right) \operatorname{td}(Y)=\iota_{*}(\operatorname{ch}(F) \operatorname{td}(C)),
$$

where $\iota_{!} F:=\sum_{i}(-1)^{i} R^{i} \iota_{*} F=\iota_{*} F$ since $R^{i} \iota_{*} F$ vanishes for $i>0$.

Remark 5.29. We define the degree of $F$ by

$$
\operatorname{deg}(F):=\chi(C, F)-\operatorname{rk}(F) \chi\left(C, \mathcal{O}_{C}\right) .
$$

Then, we have

$$
\chi(C, F)=\operatorname{deg}(F)+\operatorname{rk}(F)\left(1-p_{a}(C)\right),
$$

where $p_{a}(C)$ is the arithmetic genus of $C$. If $F$ is locally-free, $\operatorname{deg}(F)$ reduces to the ordinary degree of $F$ with (5.31) being the RiemannRoch theorem for a singular curve.

Example 5.32. Let $Z \subset X$ be a 0-dimensional subscheme of length $d$ and let $\iota: Z \hookrightarrow X$ be the inclusion. If we denote the ideal sheaf of $Z$ by $I_{Z}$ we have an exact sequence,

$$
0 \rightarrow I_{Z} \rightarrow \mathcal{O}_{X} \rightarrow \mathcal{O}_{X} / I_{Z}=\iota_{*} \mathcal{O}_{Z} \rightarrow 0
$$

Since $Q\left(\iota_{*} \mathcal{O}_{Z}\right)=(0,0, d)$ and $Q\left(\mathcal{O}_{X}\right)=([X], 0,1)$ we obtain

$$
Q\left(I_{Z}\right)=([X], 0,1-d)
$$




\subsubsection{Hilbert polynomials}

Let $H_{X}$ be an ample invertible sheaf on $X$. The Hilbert polynomial $P_{E / X}(n) \in \mathbb{Q}[n]$ of a coherent sheaf $E$ on $X$ is defined by

$P_{E / X}(n):=\chi\left(X, E \otimes H_{X}^{\otimes n}\right)=\frac{\operatorname{rk}(E) \operatorname{deg}(X)}{2} n^{2}+\operatorname{deg}(E) n+\chi(X, E)$,

where $\operatorname{deg}(E):=\left(c_{1}(E) \cup c_{1}\left(H_{X}\right)\right) \cap[X]$ and $\operatorname{deg}(X):=\operatorname{deg}\left(H_{X}\right)$. Since $[E] \mapsto P_{E / X}(n)$ is a module homomorphism, we obtain

$$
P_{F / X}(n)=P_{E / X}(n)+P_{G / X}(n),
$$

for each exact sequence (5.8) of coherent sheaves on $X$.

Let $C$ be a projective irreducible curve polarized by an ample invertible sheaf $H_{C}$ on $C$. Let $F$ be a coherent sheaf on $C$. The Hilbert polynomial of $F$ is similarly given by

$$
P_{F / C}(n):=\chi\left(C, F \otimes H_{C}^{\otimes n}\right)=\operatorname{rk}(F) \operatorname{deg}(C) n+\chi(C, F),
$$

where $\operatorname{deg}(C):=\operatorname{deg}\left(H_{C}\right)$. Suppose that we have an inclusion $\iota: C \hookrightarrow$ $X$. Since $\iota^{*} H_{X}$ is also ample, one may choose $H_{C}=\iota^{*} H_{X}$. Then it follows that

$$
P_{F / C}(n)=P_{\iota_{*} F / X}(n) .
$$

This can also be directly checked by using (5.26) and

$$
\begin{aligned}
\operatorname{deg}\left(\iota_{*} F\right) & =\operatorname{rk}(F)\left(c_{1}\left(\mathcal{O}_{X}(C)\right) \cup c_{1}\left(H_{X}\right)\right) \cap[X] \\
& =\operatorname{rk}(F) c_{1}\left(H_{X}\right) \cap \iota_{*}[C]=\operatorname{rk}(F) \operatorname{deg}\left(H_{C}\right) .
\end{aligned}
$$

Comparing (5.35) and (5.37) with the expressions of $D$-brane charges one finds that the coefficients of Hilbert polynomials are, in a sense, scalar projections of $D$-brane charges. In particular, if $D 4$-brane charges vanish, $D 0$-brane charges coincide with the constant terms of Hilbert polynomials. This fact may be a useful observation later in this section.

\subsubsection{Some moduli spaces}

Let $E$ be a coherent sheaf on $X$. Fix an ample invertible sheaf $H_{X}$ on $X$ and expand $P_{E / X}(n)$ in the form $P_{E / X}(n)=\sum_{i=0}^{\operatorname{dim}(E)} \alpha_{i}(E) n^{i} / i$ !. A 
coherent sheaf $E$ on $X$ is called semi-stable (stable) if it is pure and satisfies

$$
\frac{P_{E^{\prime} / X}(n)}{\alpha_{\operatorname{dim}\left(E^{\prime}\right)}\left(E^{\prime}\right)} \leq \frac{P_{E / X}(n)}{\alpha_{\operatorname{dim}(E)}(E)} \quad\left(\frac{P_{E^{\prime} / X}(n)}{\alpha_{\operatorname{dim}\left(E^{\prime}\right)}\left(E^{\prime}\right)}<\frac{P_{E / X}(n)}{\alpha_{\operatorname{dim}(E)}(E)}\right),
$$

for any proper subsheaf $E^{\prime} \subset E$. There is another notion of stability due to Mumford:

1. The case where $\operatorname{rk}(E)>0:$ A coherent sheaf $E$ is slope semistable (stable) if it is torsion-free and satisfies

$$
\frac{\operatorname{deg}\left(E^{\prime}\right)}{\operatorname{rk}\left(E^{\prime}\right)} \leq \frac{\operatorname{deg}(E)}{\operatorname{rk}(E)} \quad\left(\frac{\operatorname{deg}\left(E^{\prime}\right)}{\operatorname{rk}\left(E^{\prime}\right)}<\frac{\operatorname{deg}(E)}{\operatorname{rk}(E)}\right)
$$

for any subsheaf $E^{\prime}$ of $0<\operatorname{rk}\left(E^{\prime}\right)<\operatorname{rk}(E)$.

2. The case where $\operatorname{rk}(E)=0:$ A coherent sheaf $E$ is slope semistable (stable) if it is of pure dimension 1 and

$$
\frac{\chi\left(X, E^{\prime}\right)}{\operatorname{deg}\left(E^{\prime}\right)} \leq \frac{\chi(X, E)}{\operatorname{deg}(E)} \quad\left(\frac{\chi\left(X, E^{\prime}\right)}{\operatorname{deg}\left(E^{\prime}\right)}<\frac{\chi(X, E)}{\operatorname{deg}(E)}\right)
$$

for any subsheaf $E^{\prime}$ of $0<\operatorname{deg}\left(E^{\prime}\right)<\operatorname{deg}(E)$.

By (5.35) and (5.37), we have the following relations:

$$
\text { slope stable } \Rightarrow \text { stable } \Rightarrow \text { semi-stable } \Rightarrow \text { slope semi-stable. }
$$

Let $\mathcal{M}_{H_{X}}(v)$ be the moduli space of semi-stable (with respect to $\left.H_{X}\right)$ sheaves on $X$ with $D$-brane charge $v \cap[X]$. Let $\mathcal{M}_{H_{X}}^{s}(v) \subset$ $\mathcal{M}_{H_{X}}(v)$ be the subset parametrizing stable sheaves. If $\mathcal{M}_{H_{X}}^{s}(v)$ is not empty, it is smooth of dimension $\langle v, v\rangle_{X}+2$. If $v$ is primitive and $H_{X}$ is a general point of the ample cone of $X, \mathcal{M}_{H_{X}}(v)=\mathcal{M}_{H_{X}}^{s}(v)$ and $\mathcal{M}_{H_{X}}(v)$ is irreducible symplectic (hence hyperkähler). Since the choice of $H_{X}$ is not so important, we usually denote $\mathcal{M}_{H_{X}}(v)$ by $\mathcal{M}(v)$. In the following, we deal with the cases where $\mathcal{M}(v)=\mathcal{M}^{s}(v)$. If $v \cap[X]$ is expressed as $(r[X],[C], a)$, we frequently use the notation $\mathcal{M}(r, C, a)$ for $\mathcal{M}(v)$. When the isomorphism class $[E]$ of a coherent sheaf $E$ belongs 
to $\mathcal{M}(v)$, we simply write $E \in \mathcal{M}(v)$ instead of $[E] \in \mathcal{M}(v)$. For more details on $\mathcal{M}(v)$ see the original works [85-87] or an exposition [56], and for recent developments on $\mathcal{M}(v)$ see $[91,92,108,109]$.

Let $V$ be a projective scheme polarized by an ample invertible sheaf $H_{V}$. Fix a coherent sheaf $F$ on $V$. Informally speaking, the Grothendieck Quot-scheme $\operatorname{Quot}_{F / V}^{P(n)}$ parametrizes quotient sheaves of $F$ having a common Hilbert polynomial $P(n)$ or equivalently exact sequences $0 \rightarrow E \rightarrow F \rightarrow G \rightarrow 0$ such that $P_{G / V}(n)=P(n)$. If $V$ is an $S$-scheme we can similarly consider relative Quot-schemes $\operatorname{Quot}_{F / V / S}^{P(n)}$. See $[47,56]$ for more details.

Example 5.44. A fundamental case is the Hilbert scheme $X^{[d]}:=$ $\operatorname{Hilb}_{X}^{d}=$ Quot $_{\mathcal{O}_{X} / X}^{d}$ of 0 -dimensional subschemes of length $d$ in $X$. In this case relevant short exact sequences are in the form (5.33) and $X^{[d]}$ is an irreducible symplectic manifold of dimension $2 d$. If $C \subset X$ is an irreducible curve we have

$$
X^{[d]} \cong \mathcal{M}(1,0,1-d) \cong \mathcal{M}\left(1, C, p_{a}(C)-d\right),
$$

where the first isomorphism is obtained by sending a 0-dimensional subscheme $Z \subset X$ to its ideal sheaf $I_{Z}$ (cf. (5.34)) while the second one reflects the isometry (5.11) and is obtained by sending a 0 -dimensional subscheme $Z$ to $I_{Z}(C):=I_{Z} \otimes \mathcal{O}_{X}(C)$.

\subsection{D0-branes bound to a rigid smooth $D 2$-brane in a Calabi-Yau manifold}

We begin by considering a single $D 2$-brane wrapping around a fixed closed (nonsingular) Riemann surface $C_{h}$ of genus $h$ so that the worldvolume of the $D 2$-brane is $C_{h} \times \mathbb{R}$ with the time running in the direction of $\mathbb{R}$. Let us imagine that this $D 2$-brane is bound to collections of $D 0$-branes. Taking into account the fact that $D 0$-branes are the pure magnetic sources as seen from the $D 2$ brane, we may regard $D 0$-branes as vortices. To concretely realize vortices one may consider, as the effective world-volume theory of the combined system, $N=2$ abelian Higgs model [26] or more generally $N=2$ abelian Born-Infeld type theory [21] on $C_{h} \times \mathbb{R}$. The precise form of the effective theory does not matter since the BPS conditions are universal [21] and are given by 
the so-called (abelian) vortex equations on $C_{h}$. Thus the moduli space of the relative configuration of $D 0$-branes with respect to the fixed $D 2$ brane should coincide with the moduli space of vortices.

The mathematics of the vortex equations on closed Riemann surfaces has been much investigated in the literature $[14,15]^{4}$. We now review this subject rather in detail since it is conceptually important in what follows.

Suppose that a hermitian $C^{\infty}$ line bundle (i.e. $U(1)$-bundle) $L \rightarrow$ $C_{h}$ is given. Let $\mathcal{A}$ be the space of unitary connections on $L$ and $\Omega$ the space of $C^{\infty}$ sections of $L$. Our convention is such that $\sqrt{-1} A$ is a real-valued 1-form on $C_{h}$ if $A \in \mathcal{A}$. The curvature two-form is given by $F_{A}=d A$ and the covariant derivative $D_{A}=d+A$ can be decomposed as $D_{A}=\partial_{A}+\bar{\partial}_{A}$ where $\partial_{A}$ and $\bar{\partial}_{A}$ are respectively the $(1,0)$ and $(0,1)$ part of $D_{A}$. Since $\bar{\partial}_{A}$ determines a holomorphic structure on $L$, we can view $\mathcal{A}$ as the space of holomorphic structures on $L$.

Let $\omega$ denote the Kähler form on $C_{h}$. Then the vortex equations are the equations for $(A, \phi) \in \mathcal{A} \times \Omega$ given by:

$$
\begin{aligned}
& \bar{\partial}_{A} \phi=0, \\
& \Lambda_{\omega} F_{A}-\sqrt{-1}\left(|\phi|^{2}-c^{2}\right)=0,
\end{aligned}
$$

where $\Lambda_{\omega}$ is the adjoint of $\wedge \omega$ and $c$ is a real constant. The first equation of (5.46) means that the section $\phi$ is holomorphic with respect to the holomorphic structure determined by $\bar{\partial}_{A}$. Thus, in order to have a solution for $\phi$ we must have $d:=\operatorname{deg}(L) \geq 0$. The integration of the second equation of (5.46) gives the stability condition

$$
d<\frac{c^{2}}{2 \pi} \operatorname{Area}\left(C_{h}\right)
$$

which is necessary for the existence of solutions. The sufficiency was also shown in [14].

The space $\mathcal{A} \times \Omega$ is equipped with a natural Kähler, hence symplectic structure. The action of the $U(1)$ gauge group $\mathcal{G}$ on $\mathcal{A} \times \Omega$ is symplectic and has a moment map given by $\mu(A, \phi)=\Lambda_{\omega} F_{A}-\sqrt{-1}|\phi|^{2}$. Let $\mathcal{S}=\left\{(A, \phi) \in \mathcal{A} \times \Omega \mid \phi \not \equiv 0\right.$ and $\left.\bar{\partial}_{A} \phi=0\right\}$ be the set of solutions to

\footnotetext{
${ }^{4}$ The vortex equations have also appeared as the BRST fixed configurations in Witten's analysis of two dimensional linear sigma models [105].
} 
the first equation of the vortex equations. Then the moduli space of vortices is given by the symplectic quotient

$$
\left\{\mu^{-1}\left(-\sqrt{-1} c^{2}\right) \cap \mathcal{S}\right\} / \mathcal{G} .
$$

The complex gauge group $\mathcal{G}^{\mathbb{C}}$ acts on $\mathcal{A} \times \Omega$ leaving $\mathcal{S}$ invariant. We can identify the complex quotient

$$
\mathcal{S} / \mathcal{G}^{\mathbb{C}}
$$

with the set of effective divisors of degree $d$ on $C_{h}$, hence with the $d^{\text {th }}$ symmetric product $C_{h}^{(d)}$ which is a smooth $d$ dimensional Kähler manifold. This is so since every nonzero holomorphic section of an invertible sheaf determines an effective divisor and vice versa up to scalars. Indeed, there is a natural morphism (the Abel-Jacobi map)

$$
\mathscr{A}^{d}: C_{h}^{(d)} \rightarrow \operatorname{Pic}_{C_{h}}^{d}
$$

taking an effective divisor $D$ of degree $d$ to the invertible sheaf $\mathcal{O}_{C_{h}}(D)$ such that every fiber $\left(\mathscr{A}^{d}\right)^{-1}\left(\mathcal{O}_{C_{h}}(D)\right)$ is a projective space $\mathbb{P} H^{0}\left(C_{h}\right.$, $\left.\mathcal{O}_{C_{h}}(D)\right) \cong|D|$. In other words,

$$
C_{h}^{(d)} \cong\left\{(L, U) \mid L \in \operatorname{Pic}_{C_{h}}^{d}, U \subset H^{0}\left(C_{h}, L\right), \operatorname{dim} U=1\right\} .
$$

Let $K$ be a canonical divisor of $C_{h}$. If $d>2 h-2$, the morphism $\mathscr{A}^{d}$ makes $C_{h}^{(d)}$ a projective bundle over $\operatorname{Pic}_{C_{h}}^{d}$ since

$\operatorname{Ext}^{1}\left(\mathcal{O}_{C_{h}}(-D), \mathcal{O}_{C_{h}}\right) \cong H^{1}\left(C_{h}, \mathcal{O}_{C_{h}}(D)\right) \cong H^{0}\left(C_{h}, \mathcal{O}_{C_{h}}(K-D)\right)^{*}=0$,

so that we have $\operatorname{dim} H^{0}\left(C_{h}, \mathcal{O}_{C_{h}}(D)\right)=d+1-h$ by the RiemannRoch theorem. This can be rephrased in the following way. Let $\mathcal{P}$ be the Poincaré line bundle over $\operatorname{Pic}_{C_{h}}^{d} \times C_{h}$ with $\left.\mathcal{P}\right|_{\{L\} \times C_{h}} \cong L$ for every $L \in \operatorname{Pic}_{C_{h}}^{d}$ and let $\nu: \operatorname{Pic}_{C_{h}}^{d} \times C_{h} \rightarrow \operatorname{Pic}_{C_{h}}^{d}$ be the projection. Then, if $d>2 h-2, \nu_{*} \mathcal{P}$ is a vector bundle of rank $d+1-h$ and we have $C_{h}^{(d)} \cong \mathbb{P}\left(\nu_{*} \mathcal{P}\right)$.

If $d \geq 0$ and the stability condition is satisfied, the two quotients (5.48) and (5.49) are isomorphic. This is a story familiar in the context of the Kobayashi-Hitchin correspondence [74]. Therefore, the moduli space of vortices can be identified with the symmetric product $C_{h}^{(d)}$ with $d$ being the number of vortices. 
The cohomology of $C_{h}^{(d)}$ was studied by Macdonald [75]. In particular we have

$$
\sum_{d=0}^{\infty} \chi_{t, \tilde{t}}\left(C_{h}^{(d)}\right) y^{d}=\frac{(1-t y)^{h}(1-\tilde{t} y)^{h}}{(1-y)(1-t \tilde{t} y)}, \quad(|y|<1,|t \tilde{t} y|<1) .
$$

This immediately leads to

$$
\sum_{d=0}^{\infty} \chi_{t}\left(C_{h}^{(d)}\right) y^{d}=(1-t y)^{h-1}(1-y)^{h-1}, \quad(|y|<1,|t y|<1),
$$

and

$$
\sum_{d=0}^{\infty} \chi\left(C_{h}^{(d)}\right) y^{d}=(1-y)^{2 h-2}, \quad(|y|<1) .
$$

Thus we find that $\chi\left(C_{0}^{(d)}\right)=d+1$, which is consistent with the isomorphism $C_{0}^{(d)} \cong \mathbb{P}^{d}$. For $h \geq 1$ it follows that

$$
\chi\left(C_{h}^{(d)}\right)= \begin{cases}(-1)^{d}\left(\begin{array}{c}
2 h-2 \\
d
\end{array}\right) & \text { if } d \leq 2 h-2 \\
0 & \text { if } d>2 h-2\end{cases}
$$

The vanishing of $\chi\left(C_{h}^{(d)}\right)$ for $d>2 h-2$ can also be seen as follows. As mentioned $C_{h}^{(d)}$ is a projective bundle over $\mathrm{Pic}_{C_{h}}^{d}$. Since $\mathrm{Pic}_{C_{h}}^{d}$ is homeomorphic to $T^{2 h}$, we see that $\chi\left(C_{h}^{(d)}\right)=\chi\left(\mathbb{P}^{d-h}\right) \chi\left(T^{2 h}\right)=0$.

Now going back to our problem, we suppose that the smooth Riemann surface $C_{h}$ can be embedded in $X$ (or more generally a smooth Calabi-Yau manifold $Y$ ). In view of Corollary 5.24, Remark 5.29 and (5.55), the appropriate state counting function of the bound system of a $D 2$-brane wrapping once around $C_{h}$ and $D 0$-branes sticked to $C_{h}$ may be given by

$$
\sum_{d=0}^{\infty} \chi\left(C_{h}^{(d)}\right) y^{d+1-h}=\left(y^{-1 / 2}-y^{1 / 2}\right)^{2 h-2}, \quad(|y|<1) .
$$

This expression obviously enjoys the symmetry property under the exchange $y \leftrightarrow y^{-1}$. This is gratifying since the variable $y$ will be identified with the one in the previous sections and in that case the symmetry is required from the fact we are considering a closed string theory. 
Remark 5.58. The shift of $D 0$-brane charge can formally be incorporated by considering the line bundle $\tilde{L}=L \otimes \mathcal{O}_{C_{h}}(K)^{-1 / 2}$ instead of $L$ since $\operatorname{deg}(\tilde{L})=\chi\left(C_{h}, L\right)$. This twisting of the line bundle is very much reminiscent of that in the theory of the Seiberg-Witten monopole equations for 4-manifolds [106]. This should not be too much surprising since it is known that the vortex equations and the monopole equations are closely related [36]. The vortex equations can be considered as the dimensional reduction of the monopole equations. Indeed, the expression $\left(y^{-1 / 2}-y^{1 / 2}\right)^{2 h-2}$ is also equal to the Donaldson or Seiberg-Witten series of $C_{h} \times T^{2}$ [82] with the symmetry under the exchange $y \leftrightarrow y^{-1}$ being the charge conjugation symmetry of the monopole equations. For $h \geq 1$, this was shown also in [11]. There is subtlety when $h=0$ since $b_{2}^{+}\left(C_{0} \times T^{2}\right)=1$ and there is a wall-crossing phenomena ( $c f$. (3.36)) . In this case a path integral justification requires the evaluation of the $u$-plane integral [81] which has been done in [79].

Remark 5.59. Given a real 3-dimensional manifold $M$ we associate the variables $y_{i}$ to the generators of the free part of $H_{1}(M, \mathbb{Z})$. Then the Reidemeister torsion ${ }^{5} \tau\left(M ; y_{i}\right)$ of $M$ is closely related to the Alexander polynomial [97]: If $b_{1}(M)>1, \tau\left(M ; y_{i}\right)$ coincides with the Alexander polynomial $\Delta_{M}\left(y_{i}\right) \in \mathbb{Z}\left[y_{i}, y_{i}^{-1}\right]$ which can be made symmetric under the exchange $y_{i} \leftrightarrow y_{i}^{-1}$. If, on the other hand, $b_{1}(M)=1$ and $\partial M=\emptyset$, we have

$$
\tau(M ; y)=\frac{\Delta_{M}(y)}{\left(y^{-1 / 2}-y^{1 / 2}\right)^{2}}, \quad \Delta_{M}(y) \in \mathbb{Z}\left[y, y^{-1}\right],
$$

where $\Delta_{M}(y)$ is the Alexander polynomial symmetric under the exchange $y \leftrightarrow y^{-1}$. In particular, we have

$$
\tau\left(C_{h} \times S^{1} ; y\right)=\left(y^{-1 / 2}-y^{1 / 2}\right)^{2 h-2},
$$

where $y$ is associated with $\left[S^{1}\right]$. See for instance, $[18,84]$. According to Meng and Taubes [80], $\tau\left(M ; y_{i}\right)$ coincides with the Seiberg-Witten series of $M$ defined through the 3-dimensional version of the SeibergWitten monopole equations. See also a recent work [79] for the connection between the Donaldson-Witten partition function and the Reidemeister torsion. See also [33] for a relation between the Seiberg-Witten series of 4-manifolds and knot theory. It is rather curious to note that, in the following, we will encounter expressions quite similar to (5.60).

\footnotetext{
${ }^{5}$ The Reidemeister torsion is essentially equal to the Ray-Singer torsion $[18,84$, 95].
} 
Remark 5.62. Another reason for the significance of $\left(y^{-1 / 2}-y^{1 / 2}\right)^{2 h-2}$ is the following. Let $C_{h}$ be a rigid [93] smooth curve of genus $h$ in a Calabi-Yau 3-fold $Y$ where "rigid" means that the normal bundle $N=$ $N_{C_{h} / Y}$ satisfies $H^{0}\left(C_{h}, N\right)=0$. Let $p: \overline{\mathcal{C}}_{g, 0}\left(C_{h},\left[C_{h}\right]\right) \rightarrow \overline{\mathcal{M}}_{g, 0}\left(C_{h},\left[C_{h}\right]\right)$ be the universal curve and $\mu: \overline{\mathcal{C}}_{g, 0}\left(C_{h},\left[C_{h}\right]\right) \rightarrow C_{h}$ the universal evaluation map. Then it was proved in [93] that

$$
\left(y^{-1 / 2}-y^{1 / 2}\right)^{2 h-2}=(-1)^{h-1} \sum_{g=h}^{\infty} x^{2 g-2} m_{g \rightarrow h},
$$

where $y=\exp (\sqrt{-1} x)$ and

$$
m_{g \rightarrow h}:=e\left(R^{1} p_{*} \mu^{*} N\right) \cap\left[\overline{\mathcal{M}}_{g, 0}\left(C_{h},\left[C_{h}\right]\right)\right]^{\mathrm{vir}} .
$$

Note that $m_{g}$ in $\S 2$ is equal to $m_{g \rightarrow 0}$ and $m_{h \rightarrow h}=1$. Eq.(5.63) is important in the sense that it plays a key role in relating the $D 2-D 0$ state counting and the Gromov-Witten invariants.

\subsection{A $D 2$-brane moving in $K 3$}

As a warm-up for the next subsection we briefly recall the situation where a single $D 2$-brane (not bound to any $D 0$-branes) moves in $X$. This case was first studied in [107].

Let $C$ be an irreducible and reduced curve (which is not necessarily smooth) in $X$. One can consider the (component of) generalized Picard scheme $\operatorname{Pic}_{C}^{d}$ parametrizing invertible sheaves of degree $d$ on $C$ up to isomorphisms. Although $\mathrm{Pic}_{C}^{d}$ is not complete in general, one can consider its compactification $\overline{\mathrm{Pic}}_{C}^{d}$ as the set of isomorphism classes of rank-1 torsion-free sheaves of degree $d$ on $C$ where the degree of a rank1 torsion-free sheaf $L$ is defined by $\chi(C, L)-\chi\left(C, \mathcal{O}_{C}\right)$. Tensoring with an invertible sheaf of degree $k$ gives an isomorphism : $\overline{\mathrm{Pic}}_{C}^{d} \stackrel{\sim}{\rightarrow} \overline{\mathrm{Pic}}_{C}^{d+k}$.

Let $C_{h} \subset X$ be a connected nonsingular curve of genus $h$. Then the complete linear system $\left|C_{h}\right|$ is the set of all effective divisors linearly equivalent to $C_{h}$ and $\left|C_{h}\right| \cong \mathbb{P}^{h}$. The latter statement can be seen as follows. First we have $H^{2}\left(X, \mathcal{O}_{X}\left(C_{h}\right)\right) \cong H^{0}\left(X, \mathcal{O}_{X}\left(-C_{h}\right)\right)^{*}=0$ by vanishing theorem. The exact sequence $0 \rightarrow \mathcal{O}_{X} \rightarrow \mathcal{O}_{X}\left(C_{h}\right) \rightarrow \omega_{C_{h}} \rightarrow$ 0 , where $\omega_{C_{h}}:=\mathcal{O}_{C_{h}}\left(C_{h}\right)$ is a canonical sheaf on $C_{h}$, leads, by using $H^{1}\left(X, \mathcal{O}_{X}\right)=0$, to an exact sequence $0 \rightarrow H^{1}\left(X, \mathcal{O}_{X}\left(C_{h}\right)\right) \rightarrow$ 
$H^{1}\left(C_{h}, \omega_{C_{h}}\right) \rightarrow H^{2}\left(X, \mathcal{O}_{X}\right) \rightarrow 0$. Since the map $H^{1}\left(C_{h}, \omega_{C_{h}}\right) \rightarrow$ $H^{2}\left(X, \mathcal{O}_{X}\right)$ is surjective and both spaces are 1-dimensional, the kernel $H^{1}\left(X, \mathcal{O}_{X}\left(C_{h}\right)\right)$ must vanishes. Then, applying the Riemann-Roch theorem, we obtain the desired result.

Setting $\mathcal{S}_{h}:=\left|C_{h}\right|$, let $\mathcal{C}_{h} \subset \mathcal{S}_{h} \times X$ be the universal curve. For the flat family $\mathcal{C}_{h} / \mathcal{S}_{h}$ we assume that all the fibers of the structure morphism $p: \mathcal{C}_{h} \rightarrow \mathcal{S}_{h}$ are irreducible and reduced curves (of arithmetic genus $h$ ).

A $\mathcal{S}_{h}$-flat $\mathcal{O}_{\mathcal{C}_{h}}$ module $\mathcal{F}$ is called a relative rank-1 torsion-free (resp. invertible) sheaf of degree $d$ on $\mathcal{C}_{h} / \mathcal{S}_{h}$ if at each point $s \in \mathcal{S}_{h}$ the fiber $\mathcal{F}_{s}$ is a rank-1 torsion-free (resp. invertible) sheaf of degree $d$ on the fiber $\left(\mathcal{C}_{h}\right)_{s}$.

Denote by $j: \overline{\mathcal{J}}_{h}^{d} \rightarrow \mathcal{S}_{h}$ the relative compactified Picard scheme $\overline{\mathrm{Pic}}_{\mathcal{C}_{h} / \mathcal{S}_{h}}^{d} \rightarrow \mathcal{S}_{h}$ of degree $d$ which is the set of isomorphism classes of relative rank-1 torsion-free sheaves of degree $d$ on $\mathcal{C}_{h} / \mathcal{S}_{h}$.

As before, tensoring with a relative invertible sheaf of degree $k$ provides an isomorphism

$$
\sigma_{k}: \overline{\mathcal{J}}_{h}^{d} \stackrel{\sim}{\longrightarrow} \overline{\mathcal{J}}_{h}^{d+k} .
$$

Since the fibers of $p$ are Gorenstein, the relative dualizing sheaf $\omega_{\mathcal{C}_{h} / \mathcal{S}_{h}}$ is a relative invertible sheaf of degree $2 h-2$ on $\mathcal{C}_{h} / \mathcal{S}_{h}$. Thus we can use this for the construction of $\sigma_{2 h-2}$.

Also the map $F \mapsto F^{*}=\mathcal{H o m}_{\mathcal{O}_{\left(\mathcal{C}_{h}\right) s}}\left(F, \mathcal{O}_{\left(\mathcal{C}_{h}\right)_{s}}\right), s \in \mathcal{S}_{h}, F \in\left(\overline{\mathcal{J}}_{h}^{d}\right)_{s}$ determines an isomorphism

$$
\epsilon: \overline{\mathcal{J}}_{h}^{d \stackrel{\sim}{\longrightarrow}} \overline{\mathcal{J}}_{h}^{-d},
$$

Especially, if we set $\epsilon_{\omega}:=\sigma_{2 h-2} \circ \epsilon$, we obtain

$$
\epsilon_{\omega}: \overline{\mathcal{J}}_{h}^{d \stackrel{\sim}{\longrightarrow}} \overline{\mathcal{J}}_{h}^{2 h-2-d} .
$$

This map is obtained by $F \mapsto F^{\star}:=\mathcal{H o m}_{\mathcal{O}_{\left(\mathcal{C}_{h}\right) s}}\left(F,\left(\omega_{\mathcal{C}_{h} / \mathcal{S}_{h}}\right)_{s}\right)=F^{*} \otimes$ $\left(\omega_{\mathcal{C}_{h} / \mathcal{S}_{h}}\right)_{s}$. We note that $\operatorname{deg}\left(F^{*}\right)=-\operatorname{deg}(F)$ and $\chi\left(\left(\mathcal{C}_{h}\right)_{s}, F^{\star}\right)=$ $-\chi\left(\left(\mathcal{C}_{h}\right)_{s}, F\right)$.

It is known [85-87] that $\overline{\mathcal{J}}_{h}^{d}$ is an irreducible symplectic manifold of dimension $2 h$ and

$$
\overline{\mathcal{J}}_{h}^{d} \cong \mathcal{M}\left(0, C_{h}, d+1-h\right)
$$


Yau and Zaslow [107] proposed that the state counting function of a single $D 2$-brane moving in $X$ is given by

$$
\sum_{h=0}^{\infty} \chi\left(\overline{\mathcal{J}}_{h}^{0}\right) q^{h-1}=\frac{1}{\eta(\tau)^{24}} .
$$

This proposal and its implication for the enumeration of nodal rational curves in $X$ were further studied in $[5,32]$.

\section{$5.4 D$-branes bound to a $D 2$-brane moving in $K 3$}

In order to extend the results in $\S 5.2$ and describe the bound states of $D$-branes and a $D 2$-brane moving in the $K 3$ surface $X$, there are two basically different but equivalent points of view. As we saw in $\S 5.2$, the moduli spaces of vortices are isomorphic to the symmetric products of (smooth) curves. Going to the relative situation, we are led to consider relative Hilbert schemes of points on curves. This gives the first approach. On the other hand, we also observed that the moduli spaces of vortices are those of pairs consisting of line bundles on curves and their sections. This latter viewpoint can be generalized and we are led to consider the so-called coherent systems [72].

\subsubsection{Relative Hilbert schemes}

We start with the first viewpoint. We assume the same setting as in $\S 5.3$. In particular all the fibers of $p: \mathcal{C}_{h} \rightarrow \mathcal{S}_{h}$ are irreducible and reduced.

Let $X$ be polarized by an ample invertible sheaf $H_{X}$. Each fiber $\left(\mathcal{C}_{h}\right)_{s}$ is polarized by $\left(\iota_{s}\right)^{*} H_{X}$ where $\iota_{s}:\left(\mathcal{C}_{h}\right)_{s} \hookrightarrow X$ is the inclusion.

Now fix a relative rank-1 torsion-free sheaf $\mathcal{F}$ of degree $k$ on $\mathcal{C}_{h} / \mathcal{S}_{h}$. Since $\mathcal{F}$ is $\mathcal{S}_{h}$-flat and $\mathcal{S}_{h}$ is connected, the Hilbert polynomial of a fiber,

$$
P_{\mathcal{F}_{s} /\left(\mathcal{C}_{h}\right)_{s}}(n)=\operatorname{deg}\left(\left(\mathcal{C}_{h}\right)_{s}\right) n+\chi\left(\left(\mathcal{C}_{h}\right)_{s}, \mathcal{F}_{s}\right)=\operatorname{deg}\left(\left(\mathcal{C}_{h}\right)_{s}\right) n+k+1-h
$$


is constant as a function of $s \in \mathcal{S}_{h}$. Fix a positive integer $d$. Then the relative Quot-scheme $q: \operatorname{Quot}_{\mathcal{F} / \mathcal{C}_{h} / \mathcal{S}_{h}}^{d} \rightarrow \mathcal{S}_{h}$ parametrizes

$$
0 \longrightarrow E \longrightarrow \mathcal{F}_{s} \longrightarrow G \longrightarrow 0, \quad\left(s \in \mathcal{S}_{h}\right)
$$

where $E$ and $G$ are coherent sheaves on $\left(\mathcal{C}_{h}\right)_{s}$ satisfying $P_{G /\left(\mathcal{C}_{h}\right)_{s}}(n)=d$. Let $\mathcal{E}$ be the universal subsheaf and $\mathcal{G}$ the universal quotient sheaf corresponding respectively to $E$ and $G$ in (5.71):

$$
0 \longrightarrow \mathcal{E} \longrightarrow\left(q \times i d_{X}\right)^{*} \mathcal{F} \longrightarrow \mathcal{G} \longrightarrow 0 .
$$

For simplicity, we set $q^{\#} \mathcal{F}:=\left(q \times i d_{X}\right)^{*} \mathcal{F}$. Notice that

$$
P_{\mathcal{E}_{u} /\left(\mathcal{C}_{h}\right)_{q(u)}}(n)=\operatorname{deg}\left(\left(\mathcal{C}_{h}\right)_{q(u)}\right) n+k-d+1-h, \quad u \in \operatorname{Quot}_{\mathcal{F} / \mathcal{C}_{h} / \mathcal{S}_{h}}^{d} .
$$

As for the $D$-brane charges, we see that

$$
\begin{aligned}
Q\left(\left(\iota_{q(u)}\right)_{*} \mathcal{E}_{u}\right) & =\left(0,\left[C_{h}\right], k-d+1-h\right), \\
Q\left(\left(\iota_{q(u)}\right)_{*} q^{\#} \mathcal{F}_{u}\right) & =\left(0,\left[C_{h}\right], k+1-h\right), \\
Q\left(\left(\iota_{q(u)}\right)_{*} \mathcal{G}_{u}\right) & =(0,0, d)
\end{aligned}
$$

where $u \in \operatorname{Quot}_{\mathcal{F} / \mathcal{C}_{h} / \mathcal{S}_{h}}^{d}$. Note that the $D$-brane charges (5.74) are constant as functions of $u \in \operatorname{Quot}_{\mathcal{F} / \mathcal{C}_{h} / \mathcal{S}_{h}}^{d}$. This is intuitively plausible since "charges" must be conserved for a continuous family of curves.

An important case is $\mathcal{F}=\mathcal{O}_{\mathcal{C}_{h}}$. By a slight abuse of notation, we denote by $\mathcal{C}_{h}^{[d]}$ the relative Hilbert scheme $\operatorname{Hilb}_{\mathcal{C}_{h} / \mathcal{S}_{h}}^{d}=\operatorname{Quot}_{\mathcal{O}_{\mathcal{C}_{h}} / \mathcal{C}_{h} / \mathcal{S}_{h}}^{d}$ parametrizing $\mathcal{S}_{h}$-flat subschemes of $\mathcal{C}_{h}$ relatively of dimension 0 and length $d$. Obviously in this case we have $Q\left(\left(\iota_{q(u)}\right)_{*} \mathcal{E}_{u}\right)=\left(0,\left[C_{h}\right],-d+\right.$ $1-h)$. As we will see later, $\mathcal{C}_{h}^{[d]}$ is projective and smooth of dimension $d+h$.

One can construct the (degree $d$ component of) Abel-Jacobi map [1] which is the forgetful morphism

$$
\mathscr{A}_{\mathcal{F}}^{d}: \operatorname{Quot}_{\mathcal{F} / \mathcal{C}_{h} / \mathcal{S}_{h}}^{d} \longrightarrow \overline{\mathcal{J}}_{h}^{k-d}
$$

obtained by sending $u \in \operatorname{Quot}_{\mathcal{F} / \mathcal{C}_{h} / \mathcal{S}_{h}}^{d}$ to the isomorphism class of $\mathcal{E}_{u}$. (cf. (5.68) and (5.74).) The fiber of $\mathscr{A}_{\mathcal{F}}^{d}$ at $t \in \overline{\mathcal{J}}_{h}^{k-d}$ is isomorphic to $\mathbb{P H o m}_{\left(\mathcal{C}_{h}\right)_{j(t)}}\left(I, \mathcal{F}_{j(t)}\right)$ where $I$ is a rank-1 torsion-free $\mathcal{O}_{\left(\mathcal{C}_{h}\right)_{j(t)}}$-module 
representing $t$. The map $\mathscr{A}_{\mathcal{F}}^{d}$ is smooth over $t$ if $\operatorname{Ext}_{\left(\mathcal{C}_{h}\right)_{j(t)}}^{1}\left(I, \mathcal{F}_{j(t)}\right)=0$. See [1] for more details.

By tensoring with a relative invertible sheaf $\mathcal{L}$ of degree $\ell$ we obtain a commutative diagram [1]:

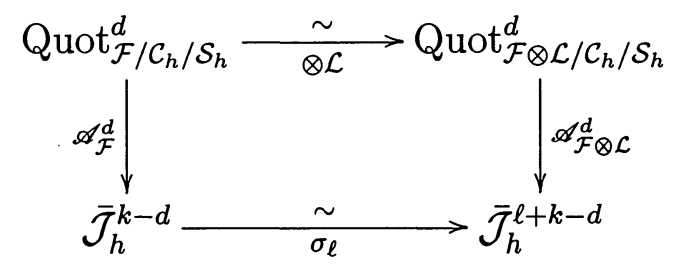

Since $\omega_{\mathcal{C}_{h} / \mathcal{S}_{h}}=: \omega$ is a relative invertible sheaf of degree $2 h-2$ (indeed it is isomorphic to $\mathcal{O}_{\mathcal{C}_{h}}\left(\mathcal{C}_{h}\right)$ ), we may take $\mathcal{L}=\omega$ in (5.76). Thus we obtain a commutative diagram

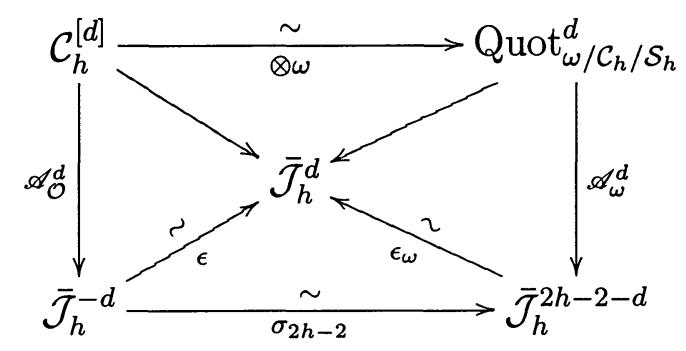

where $\mathcal{O}:=\mathcal{O}_{\mathcal{C}_{h}}$. The down diagonal arrows may be viewed as extensions of (5.50). In particular the south-east arrow $\mathcal{C}_{h}^{[d]} \rightarrow \overline{\mathcal{J}}_{h}^{d}$ is obtained by sending $u \in \mathcal{C}_{h}^{[d]}$ to (the isomorphism class of) $\mathcal{E}_{u}^{*}$ where $\mathcal{E}$ is the universal subsheaf of $\mathcal{O}$. We note that $Q\left(\left(\iota_{q(u)}\right)_{*} \mathcal{E}_{u}^{*}\right)=\left(0,\left[C_{h}\right], d+1-h\right)$.

When $\mathcal{F}=\omega$, the smoothness condition of $\mathscr{A}_{\omega}^{d}$ over $t \in \overline{\mathcal{J}}_{h}^{2 h-2-d}$ becomes

$$
\operatorname{Ext}_{\left(\mathcal{C}_{h}\right)_{j(t)}}^{1}\left(I, \omega_{j(t)}\right) \cong H^{0}\left(\left(\mathcal{C}_{h}\right)_{j(t)}, I\right)^{*}=0 .
$$

Since $\operatorname{deg}(I)=2 h-2-d$, we see that if $d>2 h-2, \mathscr{A}_{\omega}^{d}$ is smooth over every point of $\overline{\mathcal{J}}_{h}^{2 h-2-d}$. Since $\operatorname{Hom}_{\left(\mathcal{C}_{h}\right)_{j(t)}}\left(I, \omega_{j(t)}\right) \cong H^{1}\left(\left(\mathcal{C}_{h}\right)_{j(t)}, I\right)^{*}$ and $\chi\left(\left(\mathcal{C}_{h}\right)_{j(t)}, I\right)=h-1-d$, the fibers of $\mathscr{A}_{\omega}^{d}$ for $d>2 h-2$ are isomorphic to $\mathbb{P} \operatorname{Hom}_{\left(\mathcal{C}_{h}\right)_{j(t)}}\left(I, \omega_{j(t)}\right) \cong \mathbb{P}^{d-h}$. Precisely the same result holds for $\mathscr{A}_{\mathcal{O}}^{d}$ since we have the commutative diagram (5.77). We refer again to [1] for more details. 
It is natural to set $\mathcal{C}_{h}^{[0]}:=\mathcal{S}_{h} \cong \mathbb{P}^{h}$. We also have an isomorphism $\mathcal{C}_{h}^{[1]} \cong \mathcal{C}_{h}$.

With these preliminaries, we may regard $\mathcal{C}_{h}^{[d]}$ as the moduli space of the $D 2-D 0$ bound states in $X$. In order to count the $D 2-D 0$ bound states, we are naturally led to consider a combination

$$
\sum_{h=0}^{\infty} \sum_{d=0}^{\infty} \chi\left(\mathcal{C}_{h}^{[d]}\right) q^{h-1} y^{d+1-h},
$$

where we stress that the exponent of $y$ measures the $D 0$-brane charge. In the rest of this sub-subsection, we assume for every $h \geq 0$ that $C_{h}$ satisfies the condition $(\star 1)$ to be explained in $\S 5.4 .2$. Then we have

Theorem 5.80. For $0<|q|<|y|<1$,

$$
\sum_{h=0}^{\infty} \sum_{d=0}^{\infty} \chi\left(\mathcal{C}_{h}^{[d]}\right) q^{h-1} y^{d+1-h}=\frac{1}{\chi_{10,1}(\tau, \nu)}
$$

where

$$
\begin{aligned}
\chi_{10,1}(\tau, \nu) & =\eta(\tau)^{24} E(\tau, \nu)^{2} \\
& =\left(y^{-1 / 2}-y^{1 / 2}\right)^{2} q \prod_{n=1}^{\infty}\left(1-q^{n}\right)^{20}\left(1-q^{n} y\right)^{2}\left(1-q^{n} y^{-1}\right)^{2} .
\end{aligned}
$$

This result may be viewed as an amalgamation of (5.57) and (5.69). The proof of this theorem is given later when we reformulate the problem in terms of coherent systems. form:

By putting $w=q / y$ we can cast Theorem 5.80 in a more symmetric

Corollary 5.83. For $0<|w|<1,0<|y|<1$,

$$
\begin{aligned}
\sum_{h=0}^{\infty} & \sum_{d=0}^{\infty} \chi\left(\mathcal{C}_{h}^{[d]}\right) w^{h} y^{d} \\
& =\prod_{n=1}^{\infty} \frac{1}{\left(1-(w y)^{n}\right)^{20}\left(1-(w y)^{n-1} w\right)^{2}\left(1-(w y)^{n-1} y\right)^{2}} .
\end{aligned}
$$


Since the right hand side is symmetric under the exchange of $w$ and $y$ we readily obtain

\section{Corollary 5.85 (degree-genus duality).}

$$
\chi\left(\mathcal{C}_{h}^{[d]}\right)=\chi\left(\mathcal{C}_{d}^{[h]}\right) .
$$

We note that $\chi_{10,1}(\tau, \nu)$ is the (unique up to a multiplicative constant) cusp Jacobi form of weight 10 and index 1 and can alternatively be expressed in terms of the Eisenstein(-Jacobi) series [27]:

$$
\chi_{10,1}(\tau, \nu)=\frac{E_{6}(\tau) E_{4,1}(\tau, \nu)-E_{4}(\tau) E_{6,1}(\tau, \nu)}{144} .
$$

In fact $\chi_{10,1}(\tau, \nu)$ is the first Fourier-Jacobi coefficient of the Igusa cusp form of weight 10 :

$$
\chi_{10}(\Omega)=\sum_{n=1}^{\infty} q^{n} \chi_{10, n}\left(\tau^{\prime}, \nu\right),
$$

where $\Omega=\left(\begin{array}{cc}\tau & \nu \\ \nu & \tau^{\prime}\end{array}\right) \in \mathbb{H}_{2}$. The infinite product representation (5.82) has a beautiful extension to $\chi_{10}(\Omega)$ as found by Gritsenko and Nikulin [45]. They applied the exponential lifting procedure by Borcherds to a particular weak Jacobi form of weight 0 and index 1 which, as we have observed in [63], happens to be the elliptic genus of $K 3$ surfaces. For a partial review on the relations between $\chi_{10}(\Omega)$ and $K 3$ surfaces, see [64].

Now we quote the following result from $[19,28,39]$,

Lemma 5.89. For $|q|<\min \left(1,|y|,|y|^{-1}\right)$,

$$
\frac{1}{\chi_{10,1}(\tau, \nu)}=\sum_{h=0}^{\infty} q^{h-1} \frac{y^{-h} \chi_{y}\left(X^{[h]}\right)}{\left(y^{-1 / 2}-y^{1 / 2}\right)^{2}} .
$$

Remark 5.91. This is not precisely in the form presented in $[19,28,39]$ but is trivially related to it.

One may interpret this result in an alternative way since the inverse of $\chi_{10}(\Omega)$ has also a very nice expansion. Indeed, the result of [24] can be rephrased as

$$
\frac{1}{\chi_{10}(\Omega)}=\sum_{h=0}^{\infty} q^{h-1} \frac{\mathcal{E}_{X^{(h)}}^{\mathrm{orb}}\left(\tau^{\prime}, \nu\right)}{\chi_{10,1}\left(\tau^{\prime}, \nu\right)}
$$


where $\mathcal{E}_{X^{(h)}}^{\text {orb }}(\tau, \nu)$ is the orbifold elliptic genus of $X^{(h)}$. Comparing the limits $\operatorname{Im} \tau^{\prime} \rightarrow \infty$ on both sides of (5.92) one finds that

$$
\frac{1}{\chi_{10,1}(\tau, \nu)}=\sum_{h=0}^{\infty} q^{h-1} \frac{y^{-h} \chi_{y}^{\mathrm{orb}}\left(X^{(h)}\right)}{\left(y^{-1 / 2}-y^{1 / 2}\right)^{2}}
$$

where $\mathcal{E}_{X^{(h)}}^{\text {orb }}(\tau, \nu)=y^{-h} \chi_{y}^{\text {orb }}\left(X^{(h)}\right)+O(q)$. It thus follows that

\section{Lemma 5.94.}

$$
\chi_{y}^{\mathrm{orb}}\left(X^{(h)}\right)=\chi_{y}\left(X^{[h]}\right)=\chi_{y}\left(\overline{\mathcal{J}}_{h}^{d}\right)
$$

The second equality will be proved in Theorem 5.151.

Remark 5.96. Naturally we are led to the conjecture:

$$
\mathcal{E}_{X^{(h)}}^{\mathrm{orb}}(\tau, \nu)=\mathcal{E}_{X^{[h]}}(\tau, \nu)=\mathcal{E}_{\overline{\mathcal{J}}_{h}^{d}}(\tau, \nu) .
$$

Unfortunately this does not follow simply from Lemma 5.94.

Hence, as a corollary to Theorem 5.80 , we find that

Corollary 5.98. For any nonnegative integer $h$ and $|y|<1$,

$$
\begin{aligned}
\sum_{d=0}^{\infty} \chi\left(\mathcal{C}_{h}^{[d]}\right) y^{d+1-h} & =\frac{y^{-h} \chi_{y}\left(\overline{\mathcal{J}}_{h}^{d}\right)}{\left(y^{-1 / 2}-y^{1 / 2}\right)^{2}} \\
= & (h+1)\left(y^{-1 / 2}-y^{1 / 2}\right)^{2 h-2}+\cdots \\
& \quad+\chi\left(\overline{\mathcal{J}}_{h}^{d}\right)\left(y^{-1 / 2}-y^{1 / 2}\right)^{-2}
\end{aligned}
$$

where the last expression represents the expansion in $\left(y^{-1 / 2}-y^{1 / 2}\right)^{2 k-2}$ for $k=h, h-1, \ldots, 1,0$.

This should be considered as a generalization of (5.57) and it immediately implies

Corollary 5.100. For $d>2 h-2$,

$$
\chi\left(\mathcal{C}_{h}^{[d]}\right)=(d+1-h) \chi\left(\overline{\mathcal{J}}_{h}^{d}\right) .
$$


Proof. If $d>2 h-2$ the only relevant part for the calculation of $\chi\left(\mathcal{C}_{h}^{[d]}\right)$ is the term

$$
\chi\left(\overline{\mathcal{J}}_{h}^{d}\right)\left(y^{-1 / 2}-y^{1 / 2}\right)^{-2}
$$

in (5.99). Then use the series expansion (3.36) for $|y|<1$.

This result is consistent with the earlier mentioned condition for the smoothness of the Abel-Jacobi map $\mathscr{A}_{\mathcal{O}}^{d}$ since $\chi\left(\mathbb{P}^{d-h}\right)=d+1-h$. Also comparing the coefficients of $y^{1-h}$ in (5.99) one finds that $\chi\left(\mathcal{C}_{h}^{[0]}\right)=h+1$, which is consistent with $\mathcal{C}_{h}^{[0]} \cong \mathbb{P}^{h}$.

Remark 5.102. As remarked before, the expression

$$
\frac{y^{-h} \chi_{y}\left(\overline{\mathcal{J}}_{h}^{d}\right)}{\left(y^{-1 / 2}-y^{1 / 2}\right)^{2}}=\frac{y^{-h} \chi_{y}\left(X^{[h]}\right)}{\left(y^{-1 / 2}-y^{1 / 2}\right)^{2}},
$$

is reminiscent of (5.60) with the numerators playing the role of the symmetrized Alexander polynomial. We are not sure whether this is merely a coincidence or suggests the existence of certain theories on $X \times T^{2}$ or $X \times S^{1}$ which give rise to the relative versions of the SeibergWitten invariants.

\subsubsection{Coherent systems}

Now we turn to the second viewpoint of $D 2-D 0$ bound system on $X$. In the following we simplify notations by using $\langle$,$\rangle for \langle,\rangle_{X}$.

Suppose that we are given a coherent sheaf $E$ on $X$ and a vector subspace $U$ of $H^{0}(X, E) \cong \operatorname{Hom}\left(\mathcal{O}_{X}, E\right)$. The pair $(E, U)$ is called a coherent system [72]. A coherent system $(E, U)$ is called of dimension $m$ if $\operatorname{dim}(E)=m$. One may equivalently define a coherent system as a sheaf homomorphism $f: U \otimes \mathcal{O}_{X} \rightarrow E$, where $U$ is a finite dimensional vector space and $E$ is a coherent sheaf, with the property that $H^{0}(f): U \hookrightarrow H^{0}(X, E)$ is injective. Throughout this sub-subsection, we assume that $\mathcal{M}(v)$ consists of slope stable sheaves.

Remark 5.104. Assume that $v \cap[X]=(r[X],[C], a)$ with a primitive $[C]$. Then $\mathcal{M}(v)$ consists of slope stable sheaves $E$ for a general $H_{X}$ if (i) $r>0$, (ii) $r=0$ and $a \neq 0$, or (iii) $r=0$ and $|C|$ consists of irreducible and reduced members. 


\section{Let $^{6}$}

$$
\operatorname{Syst}^{n}(v):=\left\{(E, U) \mid E \in \mathcal{M}(v), U \subset H^{0}(X, E), \operatorname{dim} U=n\right\}
$$

denote the coarse moduli space of coherent systems constructed by Le Potier [72]. Thus $\operatorname{Syst}^{n}(v)$ is a projective scheme.

Definition 5.106. We set

$$
\begin{aligned}
\mathcal{M}(v)_{i} & :=\left\{E \in \mathcal{M}(v) \mid \operatorname{dim} H^{0}(X, E)=i\right\}, \\
\operatorname{Syst}^{n}(v)_{i} & :=p_{v}^{-1}\left(\mathcal{M}(v)_{i}\right),
\end{aligned}
$$

where $p_{v}: \operatorname{Syst}^{n}(v) \rightarrow \mathcal{M}(v)$ is the natural projection.

Let $C_{h}$ be an effective divisor on $X$ satisfying $C_{h}^{2}=2 h-2$. We consider the following two conditions on $C_{h}$ :

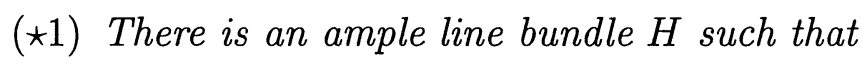

$$
C_{h} \cdot H=\min \{L \cdot H \mid L \in \operatorname{Pic}(X), L \cdot H>0\} .
$$

( $\star 2)$ Every member of $\left|C_{h}\right|$ is irreducible and reduced.

Remark 5.109. Obviously, the condition $(\star 1)$ implies the condition $(\star 2)$.

Remark 5.110. If $\operatorname{Pic}(X)=\mathbb{Z} C_{h}$ with $h>1$, then $C_{h}$ satisfies $(\star 1)$. In the moduli space of polarized $K 3$ surfaces of degree $2 h-2$, the locus of $\left(X, C_{h}\right)$ with $\operatorname{rk}(\operatorname{Pic}(X))>1$ is countable union of hypersurfaces. Hence for a general point $\left(X, C_{h}\right), \operatorname{Pic}(X)=\mathbb{Z} C_{h}$. If $\pi: X \rightarrow \mathbb{P}^{1}$ is an elliptic $K 3$ surface with a section such that $\operatorname{Pic}(X)=\mathbb{Z} \sigma \oplus \mathbb{Z} f$, where $\sigma$ is a section of $\pi$ and $f$ a fiber of $\pi$, then $C_{h}=f$ satisfies $(\star 1)$ with $h=1$ and $C_{h}=\sigma$ satisfies $(\star 1)$ with $h=0$. Indeed, $\sigma+3 f$ is ample and $f \cdot(\sigma+3 f)=\sigma \cdot(\sigma+3 f)=1$.

Remark 5.111. Under $(\star 2),\left|C_{h}\right|$ always contains a smooth curve [35, p.133-p.135].

\footnotetext{
${ }^{6} \mathrm{As}$ remarked before, we use the same notations for isomorphism classes and their representatives.
} 
Remark 5.112. Whenever we assume the condition $(\star 1)$, we use $H$ in (5.108) for the polarization of $X$.

Let $\operatorname{Gr}(k, l)$ denote the Grassmannian parametrizing $l$-dimensional vector subspaces of $\mathbb{C}^{k}$. The following is a consequence of $[108$, Lem. 2.1, Lem. 2.4]:

Lemma 5.113. Assume that $C_{h}$ satisfies $(\star 1)$ and $n \leq r$. Define $v, w \in H^{2 *}(X, \mathbb{Z})$ by $v \cap[X]=\left(r[X],\left[C_{h}\right], a\right)$ and $w \cap[X]=((r-$ $\left.n)[X],\left[C_{h}\right], a-n\right) . \quad$ Any element $f: U \otimes \mathcal{O}_{X} \rightarrow E$ of $\operatorname{Syst}^{n}(v)$ is an injection and coker $f$ is a (slope) stable sheaf. Hence we have a morphism

$$
\begin{aligned}
& q_{v}: \operatorname{Syst}^{n}(v) \quad \longrightarrow \mathcal{M}(w) \\
& \left(f: U \otimes \mathcal{O}_{X} \hookrightarrow E\right) \longmapsto \operatorname{coker} f .
\end{aligned}
$$

Moreover, by setting $m=n-(r+a)$, we obtain the following diagram:

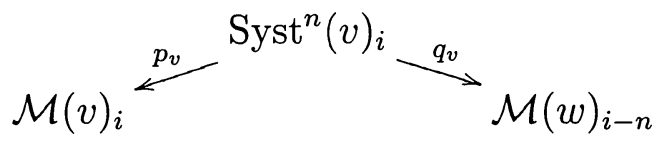

where $p_{v}$ is an étale locally trivial $\operatorname{Gr}(i, n)$-bundle and $q_{v}$ is an étale locally trivial $\operatorname{Gr}(m+i, n)$-bundle.

More precisely, we proved Lemma 2.1 in [108] under the assumption $\operatorname{Pic}(X)=\mathbb{Z} C_{h}$. Since the same proof as there works under the assumption $(\star 1)$, we obtain the diagram (5.115).

The following is well-known.

Lemma 5.116. Let $E$ be a torsion-free sheaf or a coherent sheaf of pure dimension 1 on $X$. Let $\phi: V_{0} \rightarrow E$ be a surjective homomorphism from a locally-free sheaf $V_{0}$. Then $\operatorname{ker} \phi$ is a locally-free sheaf or $\operatorname{ker} \phi=0$.

Indeed for a torsion-free or a pure dimension 1 sheaf $E$, depth $\mathcal{O}_{X, x} E_{x}$ $\geq 1$ for all point $x \in X$, where $\mathcal{O}_{X, x}$ and $E_{x}$ are the stalks of $\mathcal{O}_{X}$ and $E$ at $x$ respectively $(c f$. $[56,1.1])$. Since $X$ is smooth of dimension 2, the homological dimension $\operatorname{hd}_{\mathcal{O}_{X, x}}\left(E_{x}\right)$ of $E_{x}$ satisfies an equality $\operatorname{hd}_{\mathcal{O}_{X, x}}\left(E_{x}\right)+\operatorname{depth}_{\mathcal{O}_{X, x}} E_{x}=2$. Hence $\operatorname{hd}_{\mathcal{O}_{X, x}}\left(E_{x}\right) \leq 1$, which implies our claim. 
The next Lemma is an extension of [108, Lem 5.2].

Lemma 5.117. Under the condition $(\star 1)$, $\operatorname{Syst}^{n}(v)$ is a smooth scheme of dimension $\langle v, v\rangle+2-n\left(n+\left\langle v_{1}, v\right\rangle\right)$, where $v_{1} \cap[X]=([X], 0,1)$, namely $v_{1}=v\left(\mathcal{O}_{X}\right)$.

Proof. Let $\Lambda=(E, U)$ be a point of $\operatorname{Syst}^{n}(v)$. By He [51], the Zariski tangent space of $\operatorname{Syst}^{n}(v)$ at $\Lambda$ is given by $\operatorname{Ext}^{1}(\Lambda, \Lambda)$, the obstruction of infinitesimal liftings belong to the kernel of the composition of homomorphisms

$$
\tau: \operatorname{Ext}^{2}(\Lambda, \Lambda) \rightarrow \operatorname{Ext}^{2}(E, E) \stackrel{\operatorname{tr}}{\rightarrow} H^{2}\left(X, \mathcal{O}_{X}\right)
$$

and

$$
\operatorname{Ext}^{2}(\Lambda, \Lambda) \cong \operatorname{Ext}^{2}\left(U \otimes \mathcal{O}_{X} \rightarrow E, E\right),
$$

where $\operatorname{Ext}^{*}\left(U \otimes \mathcal{O}_{X} \rightarrow E, *\right)$ is the hypercohomology associated to the complex $U \otimes \mathcal{O}_{X} \rightarrow E$. Moreover there is an exact sequence

$$
\begin{aligned}
0 \longrightarrow \operatorname{Ext}^{0}(\Lambda, \Lambda) \longrightarrow \operatorname{Hom}(E, E) \longrightarrow \operatorname{Hom}\left(U \otimes \mathcal{O}_{X}, E\right) / V \\
\longrightarrow \operatorname{Ext}^{1}(\Lambda, \Lambda) \longrightarrow \operatorname{Ext}^{1}(E, E) \longrightarrow \operatorname{Ext}^{1}\left(U \otimes \mathcal{O}_{X}, E\right) \\
\longrightarrow \operatorname{Ext}^{2}(\Lambda, \Lambda) \longrightarrow \operatorname{Ext}^{2}(E, E) \longrightarrow \mathcal{E x t}^{2}(U)=0
\end{aligned}
$$

where $V:=\operatorname{im}\left(\operatorname{Hom}\left(U \otimes \mathcal{O}_{X}, U \otimes \mathcal{O}_{X}\right) \rightarrow \operatorname{Hom}\left(U \otimes \mathcal{O}_{X}, E\right)\right)$. Then the Serre dual of $\tau$ is the composition of homomorphisms

$$
H^{0}\left(X, \mathcal{O}_{X}\right) \rightarrow \operatorname{Hom}(E, E) \hookrightarrow \mathbb{H} \operatorname{Hom}\left(E, U \otimes \mathcal{O}_{X} \rightarrow E\right)
$$

So we shall prove that $\mathbb{H} \operatorname{Hom}\left(E, U \otimes \mathcal{O}_{X} \rightarrow E\right) \cong \mathbb{C}$. Let

$$
0 \longrightarrow \mathcal{O}_{X} \otimes \operatorname{Ext}^{1}\left(E, \mathcal{O}_{X}\right)^{*} \longrightarrow G \longrightarrow E \longrightarrow 0
$$

be the universal extension, i.e. the extension class corresponds to the identity element in

$$
\operatorname{End}\left(\operatorname{Ext}^{1}\left(E, \mathcal{O}_{X}\right)\right) \cong \operatorname{Ext}^{1}\left(E, \mathcal{O}_{X} \otimes \operatorname{Ext}^{1}\left(E, \mathcal{O}_{X}\right)^{*}\right) .
$$

We set $i:=\operatorname{dim} \operatorname{Ext}^{1}\left(E, \mathcal{O}_{X}\right)$. Since $\operatorname{dim} \mathbb{H} \operatorname{Hom}\left(E, U \otimes \mathcal{O}_{X} \rightarrow E\right) \geq 1$ by (5.121), it is sufficient to prove that

(1) $\mathbb{H o m}\left(E, U \otimes \mathcal{O}_{X} \rightarrow E\right) \longrightarrow \mathbb{H} \operatorname{Hom}\left(G, U \otimes \mathcal{O}_{X} \rightarrow E\right)$ is injective, 
(2) $\mathbb{H} \operatorname{lom}\left(G, U \otimes \mathcal{O}_{X} \rightarrow E\right) \cong \mathbb{C}$.

- Proof of (1): Since there is an exact sequence

$$
\begin{aligned}
\mathbb{E x t}^{-1}\left(\mathcal{O}_{X}^{\oplus i}, U \otimes \mathcal{O}_{X} \rightarrow E\right) \longrightarrow & \mathbb{H o m}\left(E, U \otimes \mathcal{O}_{X} \rightarrow E\right) \\
& \longrightarrow \mathbb{H} \operatorname{Hom}\left(G, U \otimes \mathcal{O}_{X} \rightarrow E\right)
\end{aligned}
$$

it is sufficient to prove that $\operatorname{Ext}^{-1}\left(\mathcal{O}_{X}^{\oplus i}, U \otimes \mathcal{O}_{X} \rightarrow E\right)=0$. We note that

$\operatorname{Ext}^{-1}\left(\mathcal{O}_{X}^{\oplus i}, U \otimes \mathcal{O}_{X} \rightarrow E\right)=\operatorname{ker}\left(\operatorname{Hom}\left(\mathcal{O}_{X}^{\oplus i}, U \otimes \mathcal{O}_{X}\right) \rightarrow \operatorname{Hom}\left(\mathcal{O}_{X}^{\oplus i}, E\right)\right)$

Since $U$ is a subspace of $\operatorname{Hom}\left(\mathcal{O}_{X}, E\right), \mathbb{E x t}^{-1}\left(\mathcal{O}_{X}^{\oplus i}, U \otimes \mathcal{O}_{X} \rightarrow E\right)=0$. Hence (1) holds.

- Proof of (2): It follows from [108, Thm. 2.5] that $G \in \mathcal{M}(v+$ $\left.i v_{1}\right)_{-\left\langle v_{1}, v+i v_{1}\right\rangle}$, i.e. $H^{1}(X, G)=0$. Hence $\operatorname{Ext}^{1}\left(G, \mathcal{O}_{X}\right)=0$ by Serre duality. By the stability of $G$, we also have $\operatorname{Hom}\left(G, \mathcal{O}_{X}\right)=0$. By the exact sequence

$$
\begin{aligned}
\operatorname{Hom}(G, U & \left.\otimes \mathcal{O}_{X}\right) \longrightarrow \operatorname{Hom}(G, E) \\
& \longrightarrow \mathbb{H} \operatorname{Hom}\left(G, U \otimes \mathcal{O}_{X} \rightarrow E\right) \longrightarrow \operatorname{Ext}^{1}\left(G, U \otimes \mathcal{O}_{X}\right),
\end{aligned}
$$

$\operatorname{Hom}(G, E) \cong \mathbb{H} \operatorname{Hom}\left(G, U \otimes \mathcal{O}_{X} \rightarrow E\right)$. Since $\operatorname{Hom}(G, E)$ fits in an exact sequence

$$
\operatorname{Hom}\left(G, \mathcal{O}_{X}^{\oplus i}\right) \longrightarrow \operatorname{Hom}(G, G) \longrightarrow \operatorname{Hom}(G, E) \longrightarrow \operatorname{Ext}^{1}\left(G, \mathcal{O}_{X}^{\oplus i}\right),
$$

and $\operatorname{Hom}(G, G) \cong \mathbb{C}$, we have $\operatorname{Hom}(G, E) \cong \mathbb{C}$. Thus (2) holds.

The proposition below was first shown by Markman [77, Thm. 39]. Proposition 5.128. Assume that $C_{h}$ satisfies the condition $(\star 1)$. For $n \geq r$, we have an isomorphism

$$
\delta: \operatorname{Syst}^{n}\left(r, C_{h}, a\right) \stackrel{\sim}{\longrightarrow} \operatorname{Syst}^{n}\left(n-r, C_{h}, n-a\right) .
$$

If $n=1$ and $r=0$, then the same assertion holds under the condition $(\star 2)$. 
Proof. For a coherent system $f: U \otimes \mathcal{O}_{X} \rightarrow E$ belonging to $\operatorname{Syst}^{n}(r$, $\left.C_{h}, a\right)$, our assumptions and [108, Lem. 2.1] imply that

(i) $f$ is surjective in codimension 1 (and hence $\operatorname{dim}$ coker $f=0$ ) and $\operatorname{ker} f$ is a (slope) stable sheaf, or

(ii) $f$ is injective and coker $f$ is a (slope) stable sheaf

according as (i) $n>r$ or (ii) $n=r$. For the second case, $f$ is also generically surjective. There is an exact sequence

$$
\begin{array}{r}
0 \longrightarrow \mathcal{H o m}_{\mathcal{O}_{X}}\left(U \otimes \mathcal{O}_{X} \rightarrow E, \mathcal{O}_{X}\right) \longrightarrow \mathcal{H o m}_{\mathcal{O}_{X}}\left(E, \mathcal{O}_{X}\right) \longrightarrow \mathcal{H o m}_{\mathcal{O}_{X}}\left(U \otimes \mathcal{O}_{X}, \mathcal{O}_{X}\right) \\
\longrightarrow \mathcal{E}^{1} t_{\mathcal{O}_{X}}^{1}\left(U \otimes \mathcal{O}_{X} \rightarrow E, \mathcal{O}_{X}\right) \longrightarrow \mathcal{E} x t_{\mathcal{O}_{X}}^{1}\left(E, \mathcal{O}_{X}\right) \longrightarrow \mathcal{E x t}_{\mathcal{O}_{X}}^{1}\left(U \otimes \mathcal{O}_{X}, \mathcal{O}_{X}\right) \\
\longrightarrow \mathcal{E} x t_{\mathcal{O}_{X}}^{2}\left(U \otimes \mathcal{O}_{X} \rightarrow E, \mathcal{O}_{X}\right) \longrightarrow \mathcal{E} x t_{\mathcal{O}_{X}}^{2}\left(E, \mathcal{O}_{X}\right) \longrightarrow \mathcal{E} x t_{\mathcal{O}_{X}}^{2}\left(U \otimes \mathcal{O}_{X}, \mathcal{O}_{X}\right)
\end{array}
$$

Since $f$ is generically surjective, $\mathcal{H o m}_{\mathcal{O}_{X}}\left(E, \mathcal{O}_{X}\right) \rightarrow \mathcal{H o m}_{\mathcal{O}_{X}}(U \otimes$ $\left.\mathcal{O}_{X}, \mathcal{O}_{X}\right)$ is injective. Hence we obtain $\mathcal{H o m}_{\mathcal{O}_{X}}\left(U \otimes \mathcal{O}_{X} \rightarrow E, \mathcal{O}_{X}\right)=0$. Since $E$ is torsion-free or of pure dimension 1, Lemma 5.116 implies that $\mathcal{E}_{x} t_{\mathcal{O}_{X}}^{2}\left(E, \mathcal{O}_{X}\right)=0$. Since $U \otimes \mathcal{O}_{X}$ is a free module, $\mathcal{E}_{x} t_{\mathcal{O}_{X}}^{k}(U \otimes$

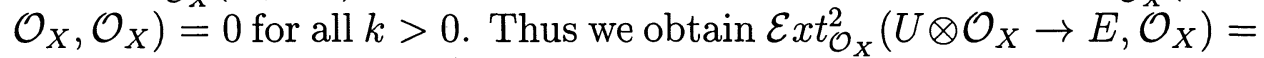
0 . We set $D(E):=\mathcal{E}_{x} t_{\mathcal{O}_{X}}^{1}\left(U \otimes \mathcal{O}_{X} \rightarrow E, \mathcal{O}_{X}\right)$. We shall prove that $D(E)$ is a (slope) stable sheaf of $v(D(E)) \cap[X]=\left((n-r)[X],\left[C_{h}\right], n-a\right)$. We first compute $v(D(E))$ : In the Grothendieck group $K_{0}(X)$, we have

$$
\begin{aligned}
\sum_{i}(-1)^{i} \mathcal{E} x t_{\mathcal{O}_{X}}^{i}\left(U \otimes \mathcal{O}_{X} \rightarrow E, \mathcal{O}_{X}\right) \\
=\sum_{i}(-1)^{i} \mathcal{E} x t_{\mathcal{O}_{X}}^{i}\left(E, \mathcal{O}_{X}\right)-\sum_{i}(-1)^{i} \mathcal{E} x t_{\mathcal{O}_{X}}^{i}\left(U \otimes \mathcal{O}_{X}, \mathcal{O}_{X}\right)
\end{aligned}
$$

For $\left(a, c_{1}, b\right) \in H^{2 *}(X, \mathbb{Z})$, we set $\left(a, c_{1}, b\right)^{*}:=\left(a,-c_{1}, b\right)$. Then we get

$$
\begin{gathered}
v\left(\sum_{i}(-1)^{i} \mathcal{E} x t_{\mathcal{O}_{X}}^{i}\left(E, \mathcal{O}_{X}\right)\right)=v(E)^{*} \\
v\left(\sum_{i}(-1)^{i} \mathcal{E} x t_{\mathcal{O}_{X}}^{i}\left(U \otimes \mathcal{O}_{X}, \mathcal{O}_{X}\right)\right)=v\left(U \otimes \mathcal{O}_{X}\right)^{*} .
\end{gathered}
$$

Hence we see that $v(D(E)) \cap[X]=\left((n-r)[X],\left[C_{h}\right], n-a\right)$. We next show that $D(E)$ is (slope) stable: By using the diagram

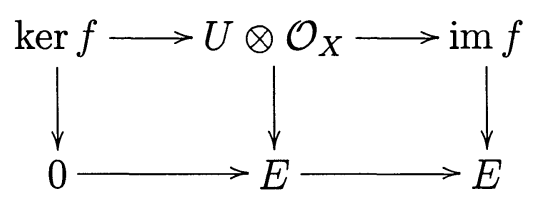


we have an exact sequence

$$
\begin{aligned}
0 \longrightarrow \mathcal{E} x t_{\mathcal{O}_{X}}^{1}\left(\operatorname{coker} f, \mathcal{O}_{X}\right) \longrightarrow D(E) \longrightarrow \mathcal{H o m}_{\mathcal{O}_{X}}\left(\operatorname{ker} f, \mathcal{O}_{X}\right) \\
\longrightarrow \mathcal{E} x t_{\mathcal{O}_{X}}^{2}\left(\operatorname{coker} f, \mathcal{O}_{X}\right) \longrightarrow 0
\end{aligned}
$$

Hence $D(E)$ is torsion-free or of pure dimension 1 according as $n>r$ or $n=r$. If $n>r$, then $\operatorname{ker} f$ is a (slope) stable vector bundle. Hence $(\operatorname{ker} f)^{*}$ is also stable, which implies that $D(E)$ is also (slope) stable. Thus $g: U^{*} \otimes \mathcal{O}_{X} \rightarrow D(E)$ is an element of $\operatorname{Syst}^{n}\left(n-r, C_{h}, n-a\right)$. If $n=r$, then ker $f=0$, and hence $D(E) \cong \mathcal{E}_{x} t_{\mathcal{O}_{X}}^{1}\left(\operatorname{coker} f, \mathcal{O}_{X}\right)$. Since $\operatorname{Supp}(\operatorname{coker} f)$ is irreducible and reduced, $D(E)$ is a stable sheaf. Therefore $g: U^{*} \otimes \mathcal{O}_{X} \rightarrow D(E)$ also belongs to $\operatorname{Syst}^{n}\left(n-r, C_{h}, n-a\right)$. Hence we obtain a map

$$
\delta: \operatorname{Syst}^{n}\left(r, C_{h}, a\right) \longrightarrow \operatorname{Syst}^{n}\left(n-r, C_{h}, n-a\right) .
$$

We shall prove that this map is holomorphic. For this purpose, we consider a family $\mathbf{f}: \mathcal{U} \otimes \mathcal{O}_{X} \rightarrow \mathcal{E}$ of coherent systems parametrized by a scheme $S$ such that $\mathcal{E}$ is flat over $S$ and $\mathcal{U}$ is a vector bundle of rank $n$ on $S$. Let $\lambda: \mathcal{W}_{0} \rightarrow \mathcal{E}$ be a surjective homomorphism from a locally-free sheaf $\mathcal{W}_{0}$ to $\mathcal{E}$. We set $\mathcal{W}_{1}:=\operatorname{ker}\left(\mathcal{W}_{0} \oplus \mathcal{U} \otimes \mathcal{O}_{X} \rightarrow \mathcal{E}\right)$. Since $\mathcal{E}_{s}, s \in S$ is torsion-free or a coherent sheaf of pure dimension 1, Lemma 5.116 implies that $\mathcal{W}_{1}$ is a locally-free sheaf. We consider a homomorphism $\psi: \mathcal{W}_{1} \oplus \mathcal{U} \otimes \mathcal{O}_{X} \rightarrow \mathcal{W}_{0} \oplus \mathcal{U} \otimes \mathcal{O}_{X}$ sending $(x, y) \in \mathcal{W}_{1} \oplus \mathcal{U} \otimes \mathcal{O}_{X}$ to $-x+y \in \mathcal{W}_{0} \oplus \mathcal{U} \otimes \mathcal{O}_{X}$, where we regard $\mathcal{W}_{1}$ and $\mathcal{U} \otimes \mathcal{O}_{X}$ as subsheaves of $\mathcal{W}_{0} \oplus \mathcal{U} \otimes \mathcal{O}_{X}$. Then we obtain a morphism of complex which is quasi-isomorphic:

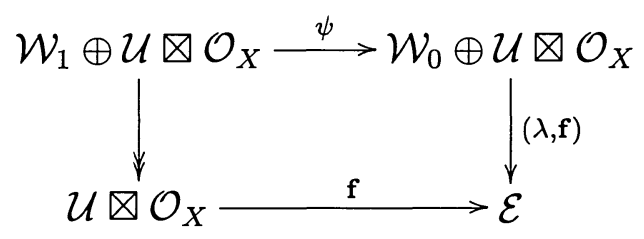

Since the construction of $\psi$ is compatible with base change and $\psi_{s}, s \in S$ is generically surjective, $\psi_{s}^{*}$ is injective, where $\psi^{*}:\left(\mathcal{W}_{0} \oplus \mathcal{U} \otimes \mathcal{O}_{X}\right)^{*} \rightarrow$ $\left(\mathcal{W}_{1} \oplus \mathcal{U} \otimes \mathcal{O}_{X}\right)^{*}$ is the dual of $\psi$. Hence coker $\psi^{*}=\mathcal{E} x t_{\mathcal{O}_{S \times X}}^{1}(\mathcal{U} \otimes$ $\left.\mathcal{O}_{X} \rightarrow \mathcal{E}, \mathcal{O}_{S \times X}\right)$ is flat over $S$ and $\left(\operatorname{coker} \psi^{*}\right)_{s} \cong \mathcal{E} x t_{\mathcal{O}_{X}}^{1}\left(\mathcal{U}_{s} \otimes \mathcal{O}_{X} \rightarrow\right.$ $\left.\mathcal{E}_{s}, \mathcal{O}_{X}\right)$. Let $\mathrm{g}: \mathcal{U}^{*} \otimes \mathcal{O}_{X} \rightarrow$ coker $\psi^{*}$ be the homomorphism induced by the natural inclusion $i: \mathcal{U}^{*} \otimes \mathcal{O}_{X} \hookrightarrow \mathcal{W}_{1}^{*} \oplus \mathcal{U}^{*} \otimes \mathcal{O}_{X}$. Then $\mathrm{g}$ : 
$\mathcal{U}^{*} \otimes \mathcal{O}_{X} \rightarrow \operatorname{coker} \psi^{*}$ is a family of coherent systems. Therefore $\delta$ is a holomorphic map. In the same way, we can construct a holomorphic $\operatorname{map} \delta^{\prime}: \operatorname{Syst}^{n}\left(n-r, C_{h}, n-a\right) \rightarrow \operatorname{Syst}^{n}\left(r, C_{h}, a\right)$. Then $\delta^{\prime}$ is the inverse of $\delta$. Indeed, by using the diagram

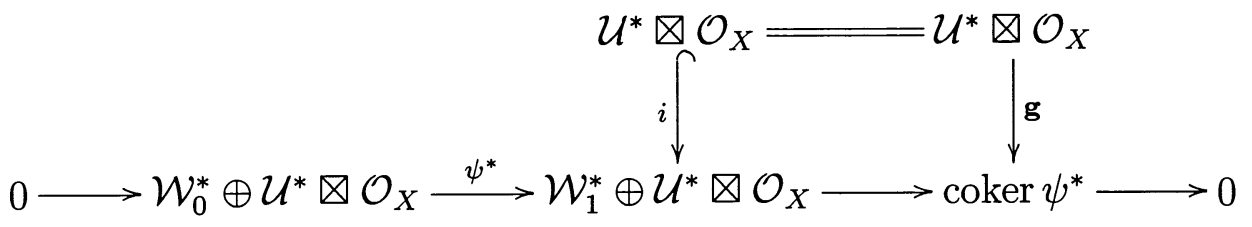

we obtain the following diagram

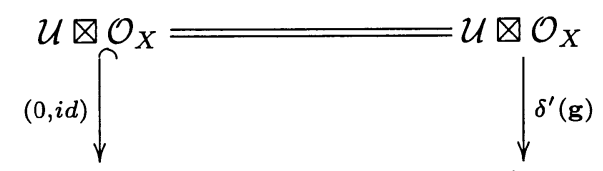

$0 \longrightarrow \mathcal{W}_{1} \oplus \mathcal{U} \otimes \mathcal{O}_{X} \stackrel{\left(-\psi, i^{*}\right)}{\longrightarrow}\left(\mathcal{W}_{0} \oplus \mathcal{U} \otimes \mathcal{O}_{X}\right) \oplus \mathcal{U} \otimes \mathcal{O}_{X} \longrightarrow \operatorname{coker}\left(-\psi, i^{*}\right) \longrightarrow 0$

Then we can easily show that $\delta^{\prime}(\mathbf{g}): \mathcal{U} \otimes \mathcal{O}_{X} \rightarrow \operatorname{coker}\left(-\psi, i^{*}\right)$ is identified with $\mathbf{f}: \mathcal{U} \otimes \mathcal{O}_{X} \rightarrow \mathcal{E}$. Thus $\delta^{\prime} \circ \delta=i d$. $\delta \circ \delta^{\prime}=i d$ also follows from the same argument.

Corollary 5.133. By the above isomorphism, we have the following diagram:

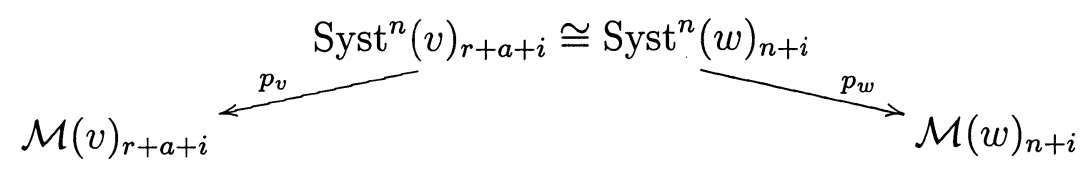

where $v \cap[X]=\left(r[X],\left[C_{h}\right], a\right)$ and $w \cap[X]=\left((n-r)[X],\left[C_{h}\right], n-a\right)$.

Proof. Let $U \otimes \mathcal{O}_{X} \rightarrow E$ be an element of $\operatorname{Syst}^{n}(v)_{r+a+i}$. Since $\mathcal{E} x t_{\mathcal{O}_{X}}^{k}\left(U \otimes \mathcal{O}_{X} \rightarrow E, \mathcal{O}_{X}\right)=0$ for $k \neq 1$, we obtain

$$
\operatorname{Ext}^{k+1}\left(U \otimes \mathcal{O}_{X} \rightarrow E, \mathcal{O}_{X}\right) \cong H^{k}\left(X, \mathcal{E} x t_{\mathcal{O}_{X}}^{1}\left(U \otimes \mathcal{O}_{X} \rightarrow E, \mathcal{O}_{X}\right)\right)
$$

Since $\mathcal{E}_{x} t_{\mathcal{O}_{X}}^{1}\left(U \otimes \mathcal{O}_{X} \rightarrow E, \mathcal{O}_{X}\right)$ is a stable sheaf of positive degree, Serre duality and (5.135) imply that

$$
\operatorname{Ext}^{3}\left(U \otimes \mathcal{O}_{X} \rightarrow E, \mathcal{O}_{X}\right)=H^{2}\left(X, \mathcal{E} x t_{\mathcal{O}_{X}}^{1}\left(U \otimes \mathcal{O}_{X} \rightarrow E, \mathcal{O}_{X}\right)\right)=0
$$


By using the canonical exact sequence

$$
\begin{aligned}
0=\operatorname{Ext}^{1}\left(U \otimes \mathcal{O}_{X}, \mathcal{O}_{X}\right) & \longrightarrow \operatorname{Ext}^{2}\left(U \otimes \mathcal{O}_{X} \rightarrow E, \mathcal{O}_{X}\right) \\
& \longrightarrow \operatorname{Ext}^{2}\left(E, \mathcal{O}_{X}\right) \longrightarrow \operatorname{Ext}^{2}\left(U \otimes \mathcal{O}_{X}, \mathcal{O}_{X}\right) \rightarrow 0
\end{aligned}
$$

we see that

$$
\begin{aligned}
& \operatorname{dim} H^{1}\left(X, \mathcal{E} x t_{\mathcal{O}_{X}}^{1}\left(U \otimes \mathcal{O}_{X} \rightarrow E, \mathcal{O}_{X}\right)\right) \\
& =\operatorname{dim} \operatorname{Ext}^{2}\left(U \otimes \mathcal{O}_{X} \rightarrow E, \mathcal{O}_{X}\right) \\
& =\operatorname{dim} \operatorname{Ext}^{2}\left(E, \mathcal{O}_{X}\right)-n \\
& =\operatorname{dim} H^{0}(X, E)-n=r+a+i-n
\end{aligned}
$$

Remark 5.139. We can easily generalize Lemma 5.117, Proposition 5.128 and Corollary 5.133 to $N\left(m v_{1}, v\right)$ in [108].

We now explain the equivalence between relative Hilbert schemes of points on curves and coherent systems under the condition $(\star 2)$. First we remark that

Lemma 5.140. Under the condition $(\star 2)$,

$$
\operatorname{Syst}^{1}\left(0, C_{h}, d+1-h\right) \cong \operatorname{Syst}_{\mathcal{C}_{h} / \mathcal{S}_{h}}\left(1, \overline{\mathcal{J}}_{h}^{d}\right),
$$

where

$$
\operatorname{Syst}_{\mathcal{C}_{h} / \mathcal{S}_{h}}\left(1, \overline{\mathcal{J}}_{h}^{d}\right):=\left\{\mathcal{O}_{C} \rightarrow L\left|C \in \mathcal{S}_{h}=\right| C_{h} \mid, L \in \overline{\operatorname{Pic}}_{C}^{d}\right\},
$$

is the relative moduli space of coherent systems on $p: \mathcal{C}_{h} \rightarrow \mathcal{S}_{h}$.

Proof. Let $\mathcal{L} \otimes \mathcal{O}_{X} \rightarrow \mathcal{E}$ be a family of coherent systems parametrized by a scheme $S$ such that $\mathcal{E}_{s} \in \mathcal{M}\left(0, C_{h}, d+1-h\right)$ for all $s \in S$, where $\mathcal{L}$ is a line bundle on $S$. Replacing $\mathcal{E}$ by $\left(\mathcal{L} \otimes \mathcal{O}_{X}\right)^{*} \otimes \mathcal{E}$, we may assume that $\mathcal{L}=\mathcal{O}_{S}$. We consider a locally-free resolution (Lemma 5.116)

$$
0 \longrightarrow V_{1} \stackrel{\phi}{\longrightarrow} V_{0} \longrightarrow \mathcal{E} \longrightarrow 0 .
$$

Then $\operatorname{det} \phi: \operatorname{det} V_{1} \rightarrow \operatorname{det} V_{0}$ is injective and it defines an effective Cartier divisor $\operatorname{Div}(\mathcal{E})$ on $S \times X$. $\operatorname{Div}(\mathcal{E})$ is called the scheme-theoretic 


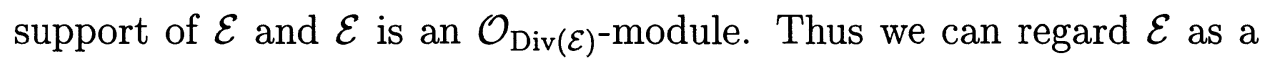
sheaf on $\operatorname{Div}(\mathcal{E})$ and we get a homomorphism $\psi: \mathcal{O}_{\operatorname{Div}(\mathcal{E})} \rightarrow \mathcal{E}$. Since the construction of $\operatorname{Div}(\mathcal{E})$ is compatible with the base change, $\operatorname{Div}(\mathcal{E})$ is flat over $S$ and $\psi_{s} \neq 0$ for all $s \in S$. Thus we get a morphism $\alpha$ : $\operatorname{Syst}^{1}\left(0, C_{h}, d+1-h\right) \rightarrow \operatorname{Syst}_{\mathcal{C}_{h} / \mathcal{S}_{h}}\left(1, \overline{\mathcal{J}}_{h}^{d}\right)$. Conversely, for a flat family of Cartier divisors $\mathcal{D} \subset S \times X$ and a family of coherent systems $\psi$ : $\mathcal{O}_{\mathcal{D}} \rightarrow \mathcal{E}, \mathcal{O}_{S \times X} \rightarrow \mathcal{O}_{\mathcal{D}} \rightarrow \mathcal{E}$ gives a family of coherent systems on $S \times X$, where we regard $\mathcal{E}$ as a sheaf on $S \times X$. Hence we have a morphism $\beta: \operatorname{Syst}_{\mathcal{C}_{h} / \mathcal{S}_{h}}\left(1, \overline{\mathcal{J}}_{h}^{d}\right) \rightarrow \operatorname{Syst}^{1}\left(0, C_{h}, d+1-h\right)$. Clearly $\beta \circ \alpha=i d$. Since every member $C \in\left|C_{h}\right|$ is irreducible and reduced, set-theoretically $\alpha \circ \beta=i d$. In particular, $\operatorname{Syst}^{1}\left(0, C_{h}, d+1-h\right)$ is isomorphic to the reduced subscheme $\operatorname{Syst}_{\mathcal{C}_{h} / \mathcal{S}_{h}}\left(1, \overline{\mathcal{J}}_{h}^{d}\right)_{\text {red }}$ of $\operatorname{Syst}_{\mathcal{C}_{h} / \mathcal{S}_{h}}\left(1, \overline{\mathcal{J}}_{h}^{d}\right)$. Therefore it is sufficient to prove that $\beta$ induces an injective homomorphism

$$
\beta_{x}: T_{x}\left(\operatorname{Syst}_{\mathcal{C}_{h} / \mathcal{S}_{h}}\left(1, \overline{\mathcal{J}}_{h}^{d}\right)\right) \longrightarrow T_{\beta(x)}\left(\operatorname{Syst}^{1}\left(0, C_{h}, d+1-h\right)\right)
$$

of Zariski tangent spaces for all $x \in \operatorname{Syst}_{\mathcal{C}_{h} / \mathcal{S}_{h}}\left(1, \overline{\mathcal{J}}_{h}^{d}\right)$. Let $\psi: \mathcal{O}_{C} \rightarrow E$ be a coherent system corresponding to a point $x \in \operatorname{Syst}_{\mathcal{C}_{h} / \mathcal{S}_{h}}\left(1, \overline{\mathcal{J}}_{h}^{d}\right)$. Assume that $\beta_{x}(\xi)=0$ for a tangent vector $\xi \in T_{x}\left(\operatorname{Syst}_{\mathcal{C}_{h} / \mathcal{S}_{h}}\left(1, \overline{\mathcal{J}}_{h}^{d}\right)\right)$. Let $\Psi: \mathcal{O}_{\mathcal{D}} \rightarrow \mathcal{E}$ be a family of coherent systems corresponding to $\xi$, where $\mathcal{D} \subset S \times X$ is a flat family of Cartier divisors over $S:=$ $\operatorname{Spec}\left(\mathbb{C}[t] /\left(t^{2}\right)\right)$. We claim that $\operatorname{Div}(\mathcal{E})=\mathcal{D}$. Then $\alpha(\beta(\Psi))=\Psi$, which implies that $\xi=0$.

- Proof of the claim: Our assumption implies that $\mathcal{E} \cong \mathcal{O}_{S} \otimes E$. In particular, $\operatorname{Div}(\mathcal{E})=S \times \operatorname{Div}(E)=S \times C$. Since $\mathcal{E}$ is generated by one element on $S \times(X \backslash \operatorname{Sing}(C))$, by the construction of $\operatorname{Div}(\mathcal{E})$, we get

$$
\operatorname{Div}(\mathcal{E})_{\mid S \times(X \backslash \operatorname{Sing}(C))}=\mathcal{D}_{\mid S \times(X \backslash \operatorname{Sing}(C))} .
$$

Since first order deformations of $\operatorname{Div}(E)=C$ are classified by $H^{0}(C$, $\left.\mathcal{O}_{C}(C)\right)$ and the map $H^{0}\left(C, \mathcal{O}_{C}(C)\right) \rightarrow H^{0}\left(C \backslash \operatorname{Sing}(C), \mathcal{O}_{C}(C)\right)$ is injective, it follows from $(5.145)$ that $\operatorname{Div}(\mathcal{E})=\mathcal{D}$.

This completes the proof of (5.141).

Remark 5.146. See Lemma 5.175 below.

Let $\mathcal{O}_{X} \rightarrow L$ be an element of $\operatorname{Syst}^{1}\left(0, C_{h}, a\right)$ and set $C:=\operatorname{Supp}(L)$. We have an exact sequence

$$
0 \longrightarrow \mathcal{O}_{X} \longrightarrow \mathcal{E} x t_{\mathcal{O}_{X}}^{1}\left(\mathcal{O}_{X} \rightarrow L, \mathcal{O}_{X}\right) \longrightarrow \mathcal{E} x t_{\mathcal{O}_{X}}^{1}\left(L, \mathcal{O}_{X}\right) \longrightarrow 0
$$


since $\mathcal{H o m}_{\mathcal{O}_{X}}\left(L, \mathcal{O}_{X}\right)=0$. Hence we obtain the following commutative diagram under the condition $(\star 2)$ :

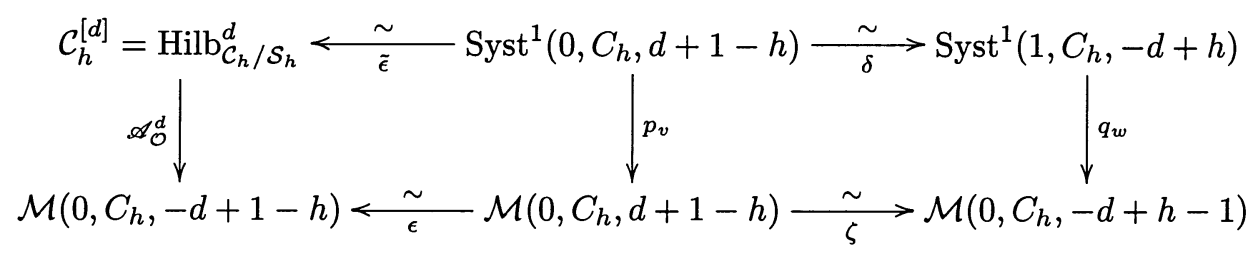

where $v \cap[X]=\left(0,\left[C_{h}\right], d+1-h\right), w \cap[X]=\left([X],\left[C_{h}\right],-d+h\right)$, and $\zeta$ and $\tilde{\epsilon}$ are isomorphisms defined by

$$
\begin{array}{cccc}
\zeta: & L & \longmapsto & \mathcal{E x t}_{\mathcal{O}_{X}}^{1}\left(L, \mathcal{O}_{X}\right), \\
\tilde{\epsilon}: & \left(\mathcal{O}_{C} \rightarrow L_{\mid C}\right) & \longmapsto & \left(\mathcal{H o m}_{\mathcal{O}_{C}}\left(L_{\mid C}, \mathcal{O}_{C}\right) \subset \mathcal{O}_{C}\right) .
\end{array}
$$

For $L \in \mathcal{M}\left(0, C_{h}, d+1-h\right), \mathcal{E} x t_{\mathcal{O}_{X}}^{1}\left(L, \mathcal{O}_{X}\right)$ is also supported on $C$ and we have

$$
\mathcal{E} x t_{\mathcal{O}_{X}}^{1}\left(L, \mathcal{O}_{X}\right)_{\mid C} \cong \mathcal{H o m}_{\mathcal{O}_{C}}\left(L_{\mid C}, \mathcal{O}_{C}\right) \otimes \omega_{C}
$$

Hence we may identify $\zeta$ with $\epsilon_{\omega}$ in (5.67). The diagram (5.148) then implies that we can also adopt $\operatorname{Syst}^{1}\left(0, C_{h}, d+1-h\right)$ as the pertinent moduli space of $D 2-D 0$ bound states.

The following was first proved by Huybrechts [55] based on the description of moduli spaces in [91]. We can find a more direct proof in [108].

Theorem 5.151. If $C_{h}$ is ample or satisfies the condition $(\star 1)$, then $\mathcal{M}\left(r, C_{h}, a\right)$ is deformation equivalent to $X^{[h-r a]}$. In particular, $\chi_{t, \tilde{t}}\left(\mathcal{M}\left(r, C_{h}, a\right)\right)=\chi_{t, \tilde{t}}\left(X^{[h-r a]}\right)$. Moreover, if $r>0$ and $\xi \in \operatorname{Pic}(X)$ is primitive, then the same assertions hold for $\mathcal{M}(r, \xi, a)$.

Proof. That the assertions hold is guaranteed by [108, Thm. 0.2] unless $r=0$ and $C_{h}$ is not ample. Hence we may assume that $r=0$ and $C_{h}$ satisfies $(\star 1)$. The following argument is very similar to the last part of the proof of [108, Thm. 3.6]. Let $H$ be an ample line bundle in (5.108). Replacing $E \in \mathcal{M}\left(0, C_{h}, a\right)$ by $E \otimes H^{\otimes n} \in \mathcal{M}\left(0, C_{h}, a+n \operatorname{deg}\left(C_{h}\right)\right)$, $n \gg 0$, we may assume that the evaluation map $\phi: H^{0}(X, E) \otimes \mathcal{O}_{X} \rightarrow$ $E$ is surjective for all $E \in \mathcal{M}\left(0, C_{h}, a\right)$. By [108, Lem. 2.1], $\operatorname{ker} \phi$ is a stable sheaf. Then the correspondence

$$
\begin{array}{ccc}
R: \mathcal{M}\left(0, C_{h}, a\right) & \longrightarrow & \mathcal{M}\left(a,-C_{h}, 0\right) \\
E & \longmapsto & \operatorname{ker} \phi
\end{array}
$$


gives an immersion. Since $\mathcal{M}\left(a,-C_{h}, 0\right)$ is irreducible (indeed deformation equivalent to $\left.X^{[h]}\right), R$ is an isomorphism. Therefore $\mathcal{M}\left(0, C_{h}, a\right)$ is also deformation equivalent to $X^{[h]}$.

Remark 5.153. The isomorphism $R$ is called the reflection by $v\left(\mathcal{O}_{X}\right)$ (cf. [86, 108]). Indeed $v\left(\mathcal{O}_{X}\right)$ is a $(-2)$-vector and $v(R(E))=$ $\operatorname{dim} H^{0}(X, E) v\left(\mathcal{O}_{X}\right)-v(E)=-\left(\left\langle v\left(\mathcal{O}_{X}\right), v(E)\right\rangle v\left(\mathcal{O}_{X}\right)+v(E)\right)$. Hence $-R(E)$ is the reflection of $v(E)$ by $v\left(\mathcal{O}_{X}\right)$. Since a $(-2)$ reflection is an important piece of the isometry group of the Mukai lattice, it is important to analyze its geometric realization.

Let us set

$$
(\xi)_{\infty}:=\prod_{n=0}^{\infty}\left(1-\xi q^{n}\right), \quad \text { and } \quad \Theta(\xi):=(\xi)_{\infty}(q / \xi)_{\infty}(q)_{\infty}
$$

For each $n \in \mathbb{Z}$ we define $\operatorname{sign}(n)$ by

$$
\operatorname{sign}(n)= \begin{cases}+1 & \text { if } n \geq 0 \\ -1 & \text { if } n<0\end{cases}
$$

Then, the following is well-known:

Lemma 5.156. For $0<|q|<\left|\xi_{1}\right|<1$ and $0<|q|<\left|\xi_{2}\right|<1$,

$$
\frac{(q)_{\infty}^{3} \Theta\left(\xi_{1} \xi_{2}\right)}{\Theta\left(\xi_{1}\right) \Theta\left(\xi_{2}\right)}=\sum_{\operatorname{sign}(i)=\operatorname{sign}(j)} \operatorname{sign}(i) q^{i j} \xi_{1}^{i} \xi_{2}^{j}
$$

Proof. See $[52,99,111]$.

Now we are in a position to state the main assertion:

Theorem 5.158. Assume that $C_{h}$ satisfies $(\star 1)$ for all $h \geq 0$. Then, for $0<|q|<|y|<1$,

$$
\begin{aligned}
& \sum_{h=0}^{\infty} \sum_{d=0}^{\infty} \chi_{t, \tilde{t}}\left(\operatorname{Syst}^{1}\left(0, C_{h}, d+1-h\right)\right)(t \tilde{t})^{1-h} q^{h-1} y^{d+1-h} \\
& =\frac{-1}{q(y)_{\infty}(q / y)_{\infty}\left((\tilde{t} \tilde{t} y)^{-1}\right)_{\infty}(t \tilde{t} y q)_{\infty}\left(t \tilde{t}^{-1} q\right)_{\infty}(q)_{\infty}^{18}\left(t^{-1} \tilde{t} q\right)_{\infty}} .
\end{aligned}
$$


In particular, by setting $t=\tilde{t}=1$, we obtain

$$
\sum_{h=0}^{\infty} \sum_{d=0}^{\infty} \chi\left(\operatorname{Syst}^{1}\left(0, C_{h}, d+1-h\right)\right) q^{h-1} y^{d+1-h}=\frac{1}{\chi_{10,1}(\tau, \nu)} .
$$

Moreover, if $C_{h}$ is ample and satisfies $(\star 2)$, then $\chi_{t, \tilde{t}}\left(\operatorname{Syst}^{1}\left(0, C_{h}, d+\right.\right.$ $1-h)$ ) is meaningful and can be obtained from (5.159) as if $C_{h}$ satisfied $(\star 1)$.

For the proof of this theorem, we need the notion of virtual Hodge polynomials. For a scheme $V$ over $\mathbb{C}$, cohomology with compact support $H_{c}^{*}(V, \mathbb{Q})$ has a natural mixed Hodge structure [22]. Let $e^{p, q}(V):=$ $\sum_{k}(-1)^{k} h^{p, q}\left(H_{c}^{k}(V)\right)$ be the virtual Hodge numbers and $e(V):=$ $\sum_{p, q} e^{p, q}(V) t^{p} \tilde{t}^{q}$ the virtual Hodge polynomial of $V$. The following properties are useful for the computation of $e(V)$. (For more details on virtual Hodge polynomials, see $[19,0.1]$.)

\section{Lemma 5.161.}

(a) Suppose that $V$ has a decomposition $V=\cup_{i=1}^{k} V_{i}$ into mutually disjoint locally closed subsets. Then

$$
e(V)=\sum_{i=1}^{k} e\left(V_{i}\right)
$$

(b) If $V$ is a smooth projective variety, then $e(V)=\chi_{t, \tilde{t}}(V)$.

For each integer $n$, we set

$$
[n]:=\frac{(t \tilde{t})^{n}-1}{t \tilde{t}-1}
$$

Then,

Lemma 5.163. Let $\pi: V \rightarrow W$ be an étale locally trivial $\mathbb{P}^{n}$-bundle over $W$. Assume that $V$ is projective over $W$. Then

$$
e(V)=[n+1] e(W) .
$$


Proof. We may assume that $W$ is smooth by applying Lemma 5.161 (a) successively. Since $\pi$ is a projective morphism, the Leray spectral sequence for $\pi$ degenerates. Moreover we obtain $R^{2} \pi_{*} \mathbb{Q} \cong \mathbb{Q}$, and hence $R^{2 i} \pi_{*} \mathbb{Q} \cong \mathbb{Q}$ for $1 \leq i \leq n$. Since $H_{c}^{*}(V, \mathbb{Q})$ is the Poincaré dual of $H^{*}(V, \mathbb{Q})$, we obtain our claim.

Proof of Theorem 5.158: By Lemma 5.117, $\operatorname{Syst}^{1}\left(0, C_{h}, d+1-h\right)$ is smooth. Hence it is sufficient to compute the virtual Hodge polynomial $e\left(\operatorname{Syst}^{1}\left(0, C_{h}, d+1-h\right)\right)$.

We start with the computation of $e\left(\operatorname{Syst}^{1}\left(r, C_{h}, a\right)\right), r+a \geq 0$. Under the condition $r+a \geq 0$, (5.115) gives the following diagram:

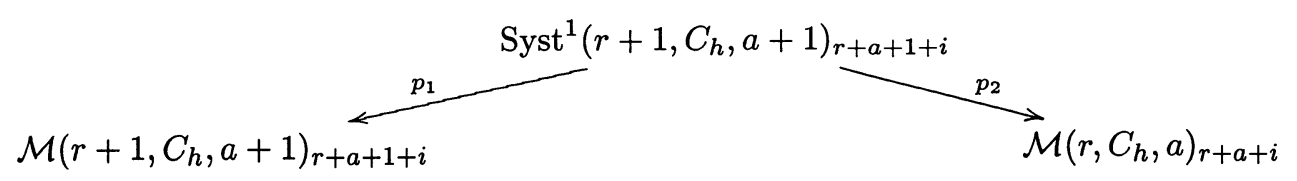

where $p_{1}$ is an étale locally trivial $\mathbb{P}^{r+a+i}$-bundle and $p_{2}$ is an étale locally trivial $\mathbb{P}^{i-1}$-bundle. By Lemma 5.163 , we have a relation

$$
\begin{aligned}
& \sum_{i \geq 0}[i] e\left(\mathcal{M}\left(r, C_{h}, a\right)_{r+a+i}\right) \\
& =\sum_{i \geq 0}[r+a+2+i] e\left(\mathcal{M}\left(r+1, C_{h}, a+1\right)_{r+a+2+i}\right) \\
& =[r+a+2] e\left(\mathcal{M}\left(r+1, C_{h}, a+1\right)\right) \\
& \quad+(t \tilde{t})^{r+a+2} \sum_{i \geq 0}[i] e\left(\mathcal{M}\left(r+1, C_{h}, a+1\right)_{r+a+2+i}\right) .
\end{aligned}
$$

Applying this successively, we see that

$$
\begin{aligned}
\sum_{i \geq 0}[i] e\left(\mathcal{M}\left(r, C_{h}, a\right)_{r+a+i}\right) \\
=[r+a+2] e\left(\mathcal{M}\left(r+1, C_{h}, a+1\right)\right) \\
\quad+(t \tilde{t})^{r+a+2} \sum_{i \geq 0}[i] e\left(\mathcal{M}\left(r+1, C_{h}, a+1\right)_{r+a+2+i}\right) \\
=\cdots \\
=[r+a+2] e\left(\mathcal{M}\left(r+1, C_{h}, a+1\right)\right) \\
\quad+(t \tilde{t})^{r+a+2}[r+a+4] e\left(\mathcal{M}\left(r+2, C_{h}, a+2\right)\right) \\
\quad+\cdots+(t \tilde{t})^{\sum_{j=1}^{k-1}(r+a+2 j)}[r+a+2 k] e\left(\mathcal{M}\left(r+k, C_{h}, a+k\right)\right)+\cdots
\end{aligned}
$$


Since

$$
e\left(\operatorname{Syst}^{1}\left(r, C_{h}, a\right)\right)=\sum_{i \geq 0}[r+a+i] e\left(\mathcal{M}\left(r, C_{h}, a\right)_{r+a+i}\right)
$$

and $\sum_{j=0}^{k-1}(r+a+2 j)=(r+a+k-1) k$, we obtain that

$$
\begin{aligned}
& e\left(\operatorname{Syst}^{1}\left(r, C_{h}, a\right)\right) \\
& =[r+a] e\left(\mathcal{M}\left(r, C_{h}, a\right)\right)+(t \tilde{t})^{r+a} \sum_{i \geq 0}[i] e\left(\mathcal{M}\left(r, C_{h}, a\right)_{r+a+i}\right) \\
& =\sum_{k \geq 0}(t \tilde{t})^{(r+a+k-1) k}[r+a+2 k] e\left(\mathcal{M}\left(r+k, C_{h}, a+k\right)\right) .
\end{aligned}
$$

Now using (5.168) with $r=0$, we find that

$$
\begin{aligned}
& \sum_{h \geq 0} \sum_{a \geq 0} e\left(\operatorname{Syst}^{1}\left(0, C_{h}, a\right)\right) y^{a}(t \tilde{t})^{1-h} q^{h-1} \\
& =\sum_{h \geq 0} \sum_{a \geq 0} \sum_{k \geq 0}(t \tilde{t})^{(a+k-1) k}[a+2 k] e\left(\mathcal{M}\left(k, C_{h}, a+k\right)\right) y^{a}(t \tilde{t})^{1-h} q^{h-1} \\
& =\sum_{h \geq 0} \sum_{j \geq i} \sum_{i \geq 0}(t \tilde{t})^{(j-1) i}[i+j] e\left(\mathcal{M}\left(i, C_{h}, j\right)\right) y^{j-i}(t \tilde{t})^{1-h} q^{h-1} \\
& =\sum_{h \geq 0} \sum_{j \geq i} \sum_{i \geq 0}(t \tilde{t})^{(j-1) i}[i+j] e\left(X^{\left[\left(C_{h}^{2}\right) / 2-i j+1\right]}\right) y^{j-i}(t \tilde{t})^{1-h} q^{h-1} \\
& =\frac{t \tilde{t}}{q}\left(\sum_{j \geq i} \sum_{i \geq 0}(t \tilde{t})^{-i}[i+j] y^{j-i} q^{i j}\right)\left(\sum_{n} e\left(X^{[n]}\right)(t \tilde{t})^{-n} q^{n}\right),
\end{aligned}
$$

where we applied Theorem 5.151 to $e\left(\mathcal{M}\left(i, C_{h}, j\right)\right)$.

For $\operatorname{Syst}^{1}\left(0, C_{h},-a\right), a>0$, we use Corollary 5.133 to find a relation

$$
\sum_{i \geq 1}[i] e\left(\mathcal{M}\left(0, C_{h},-a\right)_{i}\right)=\sum_{i \geq 1}[a+1+i] e\left(\mathcal{M}\left(1, C_{h}, 1+a\right)_{a+i+1}\right)
$$

By using (5.165) successively and performing a similar calculation as 
above, we see that

$$
\begin{aligned}
& \sum_{h \geq 0} \sum_{a>0} e\left(\operatorname{Syst}^{1}\left(0, C_{h},-a\right)\right) y^{-a}(t \tilde{t})^{1-h} q^{h-1} \\
& =\sum_{h \geq 0} \sum_{a>0} \sum_{i \geq 0}[i] e\left(\mathcal{M}\left(0, C_{h},-a\right)_{i}\right) y^{-a}(t \tilde{t})^{1-h} q^{h-1} \\
& =\sum_{h \geq 0} \sum_{a>0} \sum_{k \geq 1}(t \tilde{t})^{(a+k-1) k-a}[a+2 k] e\left(\mathcal{M}\left(k, C_{h}, a+k\right)\right) y^{-a}(t \tilde{t})^{1-h} q^{h-1} \\
& =\sum_{h \geq 0} \sum_{i>j} \sum_{j \geq 1}(t \tilde{t})^{(i-1) j-(i-j)}[i+j] e\left(\mathcal{M}\left(j, C_{h}, i\right)\right) y^{j-i}(t \tilde{t})^{1-h} q^{h-1} \\
& =\sum_{h \geq 0} \sum_{i>j} \sum_{j \geq 1}(t \tilde{t})^{(j-1) i}[i+j] e\left(X^{\left[\left(C_{h}^{2}\right) / 2-i j+1\right]}\right) y^{j-i}(t \tilde{t})^{1-h} q^{h-1} \\
& =\frac{t \tilde{t}}{q}\left(\sum_{i>j} \sum_{j \geq 1}(t \tilde{t})^{-i}[i+j] y^{j-i} q^{i j}\right)\left(\sum_{n} e\left(X^{[n]}\right)(t \tilde{t})^{-n} q^{n}\right) .
\end{aligned}
$$

Combining the above results we obtain that

$$
\begin{aligned}
& \sum_{h \geq 0} \sum_{a} e\left(\operatorname{Syst}^{1}\left(0, C_{h}, a\right)\right) y^{a}(t \tilde{t})^{1-h} q^{h-1} \\
& =\frac{t \tilde{t}}{q}\left(\sum_{i \geq 0, j>0}(t \tilde{t})^{-i}[i+j] y^{j-i} q^{i j}\right)\left(\sum_{n} e\left(X^{[n]}\right)(t \tilde{t})^{-n} q^{n}\right) \\
& =\frac{t \tilde{t}}{q(t \tilde{t}-1)}\left(\sum_{i \geq 0, j>0}\left((t \tilde{t} y)^{j} y^{-i}-(t \tilde{t} y)^{-i} y^{j}\right) q^{i j}\right)\left(\sum_{n} e\left(X^{[n]}\right)(t \tilde{t})^{-n} q^{n}\right) \\
& =\frac{-1}{q\left(1-(t \tilde{t})^{-1}\right)} \frac{(q)_{\infty}^{3} \Theta\left((t \tilde{t})^{-1}\right)}{\Theta(y) \Theta\left((t \tilde{t} y)^{-1}\right)}\left(\sum_{n} e\left(X^{[n]}\right)(t \tilde{t})^{-n} q^{n}\right),
\end{aligned}
$$

where we used Lemma 5.156 in the last step. Since

$$
\begin{aligned}
\frac{(q)_{\infty}^{3} \Theta\left((t \tilde{t})^{-1}\right)}{\Theta(y) \Theta\left((t \tilde{t} y)^{-1}\right)} & =\frac{(q)_{\infty}^{2}\left((t \tilde{t})^{-1}\right)_{\infty}(t \tilde{t} q)_{\infty}}{(y)_{\infty}(q / y)_{\infty}\left((t \tilde{t} y)^{-1}\right)_{\infty}(t \tilde{t} y q)_{\infty}} \\
& =\frac{\left(1-(t \tilde{t})^{-1}\right)(q)_{\infty}^{2}\left((t \tilde{t})^{-1} q\right)_{\infty}(t \tilde{t} q)_{\infty}}{(y)_{\infty}(q / y)_{\infty}\left((t \tilde{t} y)^{-1}\right)_{\infty}(t \tilde{t} y q)_{\infty}}
\end{aligned}
$$

and

$$
\sum_{n=0}^{\infty} e\left(X^{[n]}\right)(t \tilde{t})^{-n} q^{n}=\frac{1}{\left((t \tilde{t})^{-1} q\right)_{\infty}\left(t \tilde{t}^{-1} q\right)_{\infty}(q)_{\infty}^{20}\left(t^{-1} \tilde{t} q\right)_{\infty}(t \tilde{t} q)_{\infty}}
$$


by $[19,39]$, we reach the desired result. The last assertion of the theorem follows from the following two lemmas. ( $C f$. Remark 5.110.)

Lemma 5.175. Under the condition $(\star 2)$, $\operatorname{Syst}^{1}\left(0, C_{h}, a\right)$ is smooth of dimension $2 h+a-1$.

Proof. By Proposition 5.128, $\operatorname{Syst}^{1}\left(0, C_{h}, a\right)$ is isomorphic to $\operatorname{Syst}^{1}(1$, $\left.C_{h}, 1-a\right)$. Hence we shall prove that $\operatorname{Syst}^{1}\left(1, C_{h}, 1-a\right)$ is smooth. Let $f: \mathcal{O}_{X} \rightarrow I_{Z}(C)$ be an element of $\operatorname{Syst}^{1}\left(1, C_{h}, 1-a\right)$. Then condition $(\star 2)$ implies that $f$ is injective and $L:=\operatorname{coker} f$ is a rank-1 torsion-free sheaf when restricted to its support $C$. In order to prove the smoothness of $\operatorname{Syst}^{1}\left(1, C_{h}, 1-a\right)$ at $f: \mathcal{O}_{X} \rightarrow I_{Z}(C)$, it is sufficient to prove that $\operatorname{Hom}\left(I_{Z}(C), L\right) \cong \mathbb{C}$. Since $I_{Z}(C)_{\mid C} /$ (torsion) $\cong L_{\mid C}$ and $L$ is simple, we obtain our claim.

Lemma 5.176. Let $\left(X_{i}, H_{i}\right), i=1,2$ be polarized $K 3$ surfaces such that

(i) $H_{1}^{2}=H_{2}^{2}$.

(ii) Every member of $\left|H_{i}\right|$ is irreducible and reduced.

Then $\operatorname{Syst}^{1}\left(0, H_{1}, a\right)$ is deformation equivalent to $\operatorname{Syst}^{1}\left(0, H_{2}, a\right)$.

Proof. It is sufficient to prove the deformation equivalence of $\operatorname{Syst}^{1}(1$, $\left.H_{i}, 1-a\right)(i=1,2)$. By the connectedness of the moduli space of polarized $K 3$ surfaces, there is a family of polarized $K 3$ surfaces $\pi$ : $(\mathcal{X}, \mathcal{H}) \rightarrow S$ such that $S$ is irreducible and there are two points $s_{1}, s_{2} \in$ $S$ which satisfy $\left(\mathcal{X}_{s_{i}}, \mathcal{H}_{s_{i}}\right)=\left(X_{i}, H_{i}\right)$. Then there is a family of moduli spaces of coherent systems $\phi: \operatorname{Syst}^{1}(1, \mathcal{H}, 1-a) \rightarrow S$ such that $\operatorname{Syst}^{1}(1, \mathcal{H}, 1-a)_{s}=\operatorname{Syst}^{1}\left(1, \mathcal{H}_{s}, 1-a\right)$ and $\phi$ is a projective morphism. Assume that every member of $\left|\mathcal{H}_{s}\right|$ is irreducible and reduced for a point $s \in S$. Let $\mathcal{O}_{\mathcal{X}_{s}} \rightarrow I_{Z}\left(\mathcal{H}_{s}\right)$ be a point of $\operatorname{Syst}^{1}\left(1, \mathcal{H}_{s}, 1-a\right)$. By the proof of Lemma 5.175, $\tau: \operatorname{Ext}^{2}\left(\mathcal{O}_{\mathcal{X}_{s}} \rightarrow I_{Z}\left(\mathcal{H}_{s}\right), I_{Z}\left(\mathcal{H}_{s}\right)\right) \rightarrow$ $\operatorname{Ext}^{2}\left(I_{Z}\left(\mathcal{H}_{s}\right), I_{Z}\left(\mathcal{H}_{s}\right)\right) \rightarrow H^{2}\left(\mathcal{X}_{s}, \mathcal{O}_{\mathcal{X}_{s}}\right)$ is injective. By a standard argument, the obstruction of infinitesimal lifting lives in $\mathbb{E x t}^{2}\left(\mathcal{O}_{\mathcal{X}_{s}} \rightarrow\right.$ $\left.I_{Z}\left(\mathcal{H}_{s}\right), I_{Z}\left(\mathcal{H}_{s}\right)\right)$. Let $c_{1}(\mathcal{H}) \in R \pi_{*}^{2} \mathbb{Z}$ be the relative cohomology class of $\mathcal{H}$. Since $\mathrm{Pic}_{\mathcal{X} / S}^{c_{1}(\mathcal{H})} \rightarrow S$ is smooth (indeed isomorphic), the injectivity of $\tau$ implies that infinitesimal deformations of $\mathcal{O}_{\mathcal{X}_{s}} \rightarrow I_{Z}\left(\mathcal{H}_{s}\right)$ 
are unobstructed. Hence $\phi$ is a smooth morphism at $s$. In particular, $W:=\{s \in S \mid \phi$ is not smooth at $s\}$ is a proper closed subset of $S$. Since $s_{1}, s_{2} \in S \backslash W$ and $\phi_{\mid \phi^{-1}(S \backslash W)}$ is smooth, we obtain our claim.

\subsection{D0-branes bound to a $D 2$-brane moving in the fibers of the $K 3$ fibration}

In the above we have been studying the case where the $D 2-D 0$ bound system is moving in a fixed $K 3$ surface $X$. Similarly, we should like to investigate the case where the bound system of a single $D 2$-brane and collections of $D 0$-branes is moving in the fibers of the $K 3$-fibration $\pi_{1}: Y \rightarrow W_{1}$ described in $\S 4$. This is not an easy task in general since the details depend on the choice of $Y$ and we do not have good control of the relevant moduli spaces as in the single $K 3$ case. So, unfortunately, there is very little we can say at the moment. However, one easily sees that

$$
\Phi_{0}(\tau, z, \nu)=\frac{\Psi_{10, m}(\tau, z)}{\chi_{10,1}(\tau, \nu)} .
$$

Actually it was this observation that motivated us to consider the meaning of $1 / \chi_{10,1}(\tau, \nu)$ leading to the results in $\S 5.4$.

Remark 5.178. Let $Y_{s}$ be a generic (smooth) fiber of $\pi_{1}: Y \rightarrow W_{1}$. By our assumption, $Y_{s}$ is an elliptic $K 3$ surface with a section. Since in general $Y_{s}$ does not satisfy the conditions in $\S 5.4$, we will need a slight perturbation of the complex structure of $Y_{s}$ in order to apply the results in $\S 5.4$.

The argument given in $\S 5.4$ naturally suggests that $\Phi_{0}(\tau, z, \nu)$ counts the pertinent $D 2-D 0$ bound states in the $K 3$ fibers. An appropriate mathematical setting for justification of this would probably be again coherent systems of dimension 1 in $Y$ and their moduli spaces.

We should also remark on the following point. While we have assumed $|y|<1$ so far in this section, we previously assumed that $|y|=1$ $(y \neq 1)$ when we Fourier-expand $\Phi_{0}(\tau, z, \nu)$ in order to obtain the infinite product formula of the string partition function. This was to realize the manifest symmetry $D(n, \gamma, j)=D(n, \gamma,-j)$ and may be 
regarded as the conjugation symmetry of $D 0$-brane charge. Thus we may suppose that the Fourier coefficients $D(n, \gamma, j)$ count (with the conjugation symmetry of $D 0$-brane charge imposed) the bound states of $D 0$-branes and a $D 2$-brane moving in the fibers of $\pi_{1}: Y \rightarrow W_{1}$ where the $D 0$-brane charge is $j$ and the $D 2$-brane charge specifies $(n, \gamma)$.

The cases of several coincident D2-branes bound to collections of $D 0$-branes are presumably taken care of by the actions of Hecke operators $V_{\ell}$ on $\Phi_{0}$.

\section{Vertex operators and $D 2-D 0$ bound states}

In the previous section we encountered the expression

$$
\frac{1}{\chi_{10,1}(\tau, \nu)}=\frac{1}{\eta(\tau)^{24} E(\tau, \nu)^{2}}
$$

as the enumeration function of the $D 2-D 0$ bound states in a $K 3$ surface $X$. However, as every string theorist would readily realize, the right hand side coincides with the (unnormalized) one-loop tachyon two-point function of bosonic open string. This fact immediately leads to an anticipation that the $D 2-D 0$ bound states are related to the theory of vertex operators. In the present section we will explore this possibility although our understanding of the relation remains admittedly superficial.

Motivated by the observation in [98], Nakajima [89,90] and independently Grojnowski [46] showed that there exists a geometrical realization of the Heisenberg algebra on $\oplus_{n} H_{*}\left(X^{[n]}\right)$. See also related works $[3,71]$. It would be most desirable to have similar realizations and interpretations for what we will see below.

Almost all the technical aspects given below have been known since the era of dual resonance model [57] which was a precursor of string theory. 


\subsection{Heisenberg algebra and the Fock space repre- sentation}

Let $(\Lambda,\langle\rangle$,$) be an integral lattice of rank \ell$ and set $\mathcal{V}=\Lambda_{\mathbb{C}}$. We extend $\langle$,$\rangle by \mathbb{C}$-linearity. For each $n \in \mathbb{Z}$ let $\mathcal{V}(n)$ be a copy of $\mathcal{V}$ and set

$$
\mathrm{h}=\bigoplus_{n \neq 0} \mathcal{V}(n) \oplus \mathbb{C} \kappa, \quad \tilde{\mathrm{h}}=\bigoplus_{n \in \mathbb{Z}} \mathcal{V}(n) \oplus \mathbb{C} \kappa,
$$

where $\mathbb{C} \kappa$ is a 1 -dimensional vector space spanned by $\kappa$. For $a \in \mathcal{V}$, let $a(n)$ denote the corresponding element in $\mathcal{V}(n)$. The commutation relations

$$
[a(m), b(n)]=m\langle a, b\rangle \delta_{m,-n} \kappa, \quad[a(m), \kappa]=0,
$$

make $\mathrm{h}$ and $\tilde{\mathrm{h}}$ infinite dimensional Lie algebras with $\mathrm{h}$ being a Heisenberg algebra.

Setting $\mathrm{h}_{ \pm}=\bigoplus_{n>0} \mathcal{V}( \pm n)$, we obtain the triangular decompositions:

$$
\mathrm{h}=\mathrm{h}_{+} \oplus \mathbb{C} \kappa \oplus \mathrm{h}_{-}, \quad \tilde{\mathrm{h}}=\mathrm{h}_{+} \oplus \mathbb{C} \kappa \oplus \mathcal{V}(0) \oplus \mathrm{h}_{-} .
$$

Let $S\left(\mathrm{~h}_{-}\right)$be the symmetric algebra of $\mathrm{h}_{-}$. This is isomorphic to the $\ell$-fold tensor product of the polynomial ring $\mathbb{C}\left[x_{1}, x_{2}, \ldots\right]$ in infinitely many commuting variables $x_{1}, x_{2}, \ldots$ The Fock space $S\left(\mathrm{~h}_{-}\right)$is graded by assigning the elements of $\mathcal{V}(-n)$ the degree $n$ and it becomes an h-module in the following way. First, $a(n)\left(n \in \mathbb{Z}_{-}\right)$acts on $S\left(\mathrm{~h}_{-}\right)$by the left multiplication. For each $n \in \mathbb{Z}_{+}$let $\partial_{a(n)}: \mathrm{h}_{-} \rightarrow \mathbb{C}$ be a linear function determined by $b(-k) \mapsto n\langle a, b\rangle \delta_{n, k}$ for all $b \in \mathcal{V}$ and $k \in \mathbb{Z}_{+}$. We can uniquely extend $\partial_{a(n)}$ to a derivation on $S\left(\mathrm{~h}_{-}\right)$for which we keep the same notation. The action of $a(n)\left(n \in \mathbb{Z}_{+}\right)$on $S\left(\mathrm{~h}_{-}\right)$is given by identifying $a(n)$ with $\partial_{a(n)}$. Finally $\kappa$ acts as the identity.

Let $\mathbb{C}[\Lambda]$ be the group algebra with linear basis $\left\{e^{\alpha} \mid \alpha \in \Lambda\right\}$ and multiplication $e^{\alpha} e^{\beta}=e^{\alpha+\beta}$. The total Fock space $\mathfrak{F}$ is defined as

$$
\begin{aligned}
\mathfrak{F} & =S\left(\mathrm{~h}_{-}\right) \otimes \mathbb{C}[\Lambda] \\
& =\amalg_{\alpha \in \Lambda} \mathfrak{F}_{\alpha}
\end{aligned}
$$

with $\mathfrak{F}_{\alpha}=S\left(\mathrm{~h}_{-}\right) \otimes e^{\alpha}$. Then $\mathfrak{F}$ becomes an $\tilde{\mathrm{h}}$-module by letting

$$
\begin{aligned}
a(n)\left(u \otimes e^{\alpha}\right) & =(a(n) u) \otimes e^{\alpha}, \quad(n \neq 0), \\
\kappa\left(u \otimes e^{\alpha}\right) & =u \otimes e^{\alpha},
\end{aligned}
$$


and

$$
a(0)\left(u \otimes e^{\alpha}\right)=\langle a, \alpha\rangle u \otimes e^{\alpha} .
$$

Remark 6.8. It is customary to introduce the twisted group algebra $\mathbb{C}\{\Lambda\}$ instead of the group algebra $\mathbb{C}[\Lambda]$ in the standard theory of vertex operators associated with lattices. However, for the purpose of the present section the ordinary group algebra $\mathbb{C}[\Lambda]$ suffices.

The conjugate linear involution ${ }^{*}$ on $\mathrm{h}$ and $\tilde{\mathrm{h}}$ is defined through

$$
a(n)^{*}=\bar{a}(-n), \quad \kappa^{*}=\kappa,
$$

where ${ }^{-}$stands for the complex conjugation.

Then one can introduce a contravariant hermitian bilinear form $\langle\mid\rangle$ on $\mathfrak{F}$ by demanding

$$
\begin{aligned}
& \langle A u \mid v\rangle=\left\langle u \mid A^{*} v\right\rangle, \quad \text { for all } A \in \tilde{\mathrm{h}} \text { and for all } u, v \in \mathfrak{F} \\
& \left\langle 1 \otimes e^{\alpha} \mid 1 \otimes e^{\beta}\right\rangle=\delta_{\alpha, \beta}, \quad \text { for all } \alpha, \beta \in \Lambda .
\end{aligned}
$$

In particular we set $\mathbf{1}=1 \otimes e^{0}$. Some useful identities can be easily obtained;

$$
\begin{aligned}
\langle a(-n) \mid b(-n)\rangle & =n\langle\bar{a}, b\rangle \\
e^{a(n)} e^{b(-n)} & =e^{n\langle\bar{a}, b\rangle} e^{b(-n)} e^{a(n)} \\
\left\langle e^{a(-n)} \mid e^{b(-n)}\right\rangle & =e^{n\langle\bar{a}, b\rangle} \\
e^{a(n)} e^{b(-n)} \mathbf{1} & =e^{n\langle\bar{a}, b\rangle} e^{b(-n)} \mathbf{1}
\end{aligned}
$$

\subsection{The Virasoro algebra}

Let $\left\{e_{i}\right\}$ be a basis of $\mathcal{V}$ and let $\left\{e^{i}\right\}$ be the dual basis with respect to $\langle$,$\rangle so that \left\langle e^{i}, e_{j}\right\rangle=\delta_{i, j}$. We assume that $\bar{e}_{i}=e_{i}$ and $\bar{e}^{i}=e^{i}$. Then we have

$$
\sum_{i=1}^{\ell}\left\langle a, e^{i}\right\rangle\left\langle e_{i}, b\right\rangle=\langle a, b\rangle, \quad \text { for all } a, b \in \mathcal{V} .
$$

The Virasoro operators are defined for each $n \in \mathbb{Z}$ by

$$
L(n)=\frac{1}{2} \sum_{i=1}^{\ell} \sum_{m \in \mathbb{Z}}: e^{i}(n-m) e_{i}(m):
$$


where

$$
: a(n) b(m):= \begin{cases}a(n) b(m) & \text { if } n \leq m, \\ b(m) a(n) & \text { if } n>m .\end{cases}
$$

They satisfy the commutation relations of the Virasoro algebra with the central charge $\ell$ :

$$
[L(m), L(n)]=(m-n) L(m+n)+\frac{\ell}{12}\left(m^{3}-m\right) \delta_{m,-n} \kappa .
$$

Using (6.15) it is easy to see that

$$
L(0)\left(1 \otimes e^{\alpha}\right)=\frac{\|\alpha\|^{2}}{2}\left(1 \otimes e^{\alpha}\right),
$$

where $\|\alpha\|^{2}=\langle\alpha, \alpha\rangle$. It is also not difficult show that

$$
[L(m), a(n)]=-m a(m+n),
$$

from which we obtain a useful identity

$$
q^{L(0)} e^{a(-n)} q^{-L(0)}=e^{q^{n} a(-n)} .
$$

\subsection{Vertex operators}

We set

$$
\begin{aligned}
& X_{ \pm}(a, y):=\sum_{n=1}^{\infty} \frac{y^{\mp n}}{\mp n} a( \pm n) \\
& P_{ \pm}(a, y):=y \frac{d}{d y} X_{ \pm}(a, y)=\sum_{n=1}^{\infty} y^{\mp n} a( \pm n)
\end{aligned}
$$

For $\left|w_{1}\right|>\left|w_{2}\right|$ we obtain commutation relations:

$$
\begin{aligned}
{\left[X_{+}\left(a, w_{1}\right), X_{-}\left(b, w_{2}\right)\right] } & =\langle a, b\rangle \log (1-y), \\
{\left[P_{+}\left(a, w_{1}\right), P_{-}\left(b, w_{2}\right)\right] } & =\frac{\langle a, b\rangle}{\left(y^{-1 / 2}-y^{1 / 2}\right)^{2}},
\end{aligned}
$$

where $y=w_{2} / w_{1}$.

The vertex operator is defined for each $\alpha \in \Lambda$ by

$$
V(\alpha, y)=y^{\frac{\|\alpha\|^{2}}{2}} e^{X_{-}(\alpha, y)} e^{\alpha} y^{\alpha(0)} e^{X_{+}(\alpha, y)},
$$


where $e^{\alpha}\left(u \otimes e^{\beta}\right)=u \otimes e^{\alpha} e^{\beta}$ and $y^{\alpha(0)}\left(u \otimes e^{\beta}\right)=y^{\langle\alpha, \beta\rangle} u \otimes e^{\beta}$.

It follows from (6.24) that for $\left|w_{1}\right|>\left|w_{2}\right|$,

$$
\begin{aligned}
& V\left(\alpha, w_{1}\right) V\left(\beta, w_{2}\right)=w_{1}^{\frac{\|\alpha\|^{2}}{2}} w_{2}^{\frac{\|\beta\|^{2}}{2}}\left(w_{1}-w_{2}\right)^{\langle\alpha, \beta\rangle} \\
& \quad \times e^{X_{-}\left(\alpha, w_{1}\right)+X_{-}\left(\beta, w_{2}\right)} e^{\alpha+\beta} w_{1}^{\alpha(0)} w_{2}^{\beta(0)} e^{X_{+}\left(\alpha, w_{1}\right)+X_{+}\left(\beta, w_{2}\right)} .
\end{aligned}
$$

Using (6.21) we find that

$$
q^{L(0)} V(\alpha, y) q^{-L(0)}=V(\alpha, q y) .
$$

\subsection{Two-point correlation functions and $D 2-D 0$ bound states}

As in $\S 5$ let $X$ be a projective $K 3$ surface and for each $h \geq 0$ let $C_{h}$ be a smooth curve of genus $h$ on $X$ satisfying $(\star 1)$ in $\S 5.4 .2$. Then we make an identification

$$
\Lambda=H^{2 *}(X, \mathbb{Z})(-1) \cong \mathrm{E}_{8}^{\oplus 2} \oplus H(-1)^{\oplus 4}, \quad\langle,\rangle=-\langle,\rangle_{X} .
$$

(We will try to be general in the following so that most of the results are applicable to surfaces with vanishing odd cohomologies.)

The connection between the symmetric products of a smooth curve on a surface and vertex operators has been pointed out by Grojnowski [46] and further discussed by Nakajima [90]. Indeed it immediately follows from (6.27) that

$$
\langle\mathbf{1} \mid V(-\alpha, 1) V(\alpha, y) \mathbf{1}\rangle=\frac{1}{\left(y^{-1 / 2}-y^{1 / 2}\right)^{\|\alpha\|^{2}}}, \quad(|y|<1) .
$$

Consider the expansion

$$
e^{X_{-}(\alpha, y)}=\sum_{d=0}^{\infty} \alpha^{(d)} y^{d}
$$

where

$$
\alpha^{(d)}=s_{d}(\alpha(-1), \alpha(-2), \ldots, \alpha(-d)),
$$


with $s_{d}\left(x_{1}, \ldots, x_{d}\right)$ being the Schur polynomial of degree $d$. Then

$$
\begin{aligned}
\langle\mathbf{1} \mid V(-\alpha, 1) V(\alpha, y) \mathbf{1}\rangle & =y^{\frac{\|\alpha\|^{2}}{2}}\left\langle\mathbf{1} \mid e^{X_{+}(-\alpha, 1)} e^{X_{-}(\alpha, y)} \mathbf{1}\right\rangle \\
& =\sum_{m, d=0}^{\infty}\left\langle\mathbf{1} \mid\left(\alpha^{(m)}\right)^{*} \alpha^{(d)} \mathbf{1}\right\rangle y^{d+\frac{\|\alpha\|^{2}}{2}} \\
& =\sum_{d=0}^{\infty}\left\langle\alpha^{(d)} \mathbf{1} \mid \alpha^{(d)} \mathbf{1}\right\rangle y^{d+\frac{\|\alpha\|^{2}}{2}} .
\end{aligned}
$$

Take $\alpha=c_{1}\left(\mathcal{O}_{X}\left(C_{h}\right)\right)$. Since $\|\alpha\|^{2}=-C_{h} \cdot C_{h}=2-2 h$ it follows from (5.57) and (6.30) that

$$
\chi\left(C_{h}^{(d)}\right)=\left\langle\alpha^{(d)} \mathbf{1} \mid \alpha^{(d)} \mathbf{1}\right\rangle .
$$

(cf. [46] and Exercise 9.18 in [90].) What we will discuss below is a more complicated relation between the relative Hilbert schemes $\mathcal{C}_{h}^{[d]}$ and vertex operators.

Set $\xi^{\prime}=\sum_{i=1}^{\ell} \xi^{(i)} e^{i}$ for any $\xi=\sum_{i=1}^{\ell} \xi^{(i)} e_{i} \in \mathcal{V}$. Let $\mathbb{L}: \mathcal{V} \rightarrow \mathcal{V}$ be a linear map and let $\mu(\mathbb{L})$ be the $\ell$ by $\ell$ matrix whose $(i, j)$-th entry is $\left\langle e^{i}, \mathbb{L} e_{j}\right\rangle$. Suppose that $\mu(\mathbb{L})$ is diagonalizable and the real parts of its eigenvalues are positive. Then the Gaussian integral leads to

\section{Lemma 6.35.}

$$
\int d \bar{\xi} d \xi e^{-\left\langle\bar{\xi}^{\prime}, \mathbb{L} \xi\right\rangle+\langle a, \xi\rangle+\left\langle b, \bar{\xi}^{\prime}\right\rangle}=\frac{1}{\operatorname{det} \mu(\mathbb{L})} e^{\left\langle a, \mathbb{L}^{-1} b\right\rangle},
$$

where $d \bar{\xi} d \xi=\prod_{i=1}^{\ell} d \bar{\xi}^{(i)} d \xi^{(i)} /(2 \pi)^{\ell}$

The trace of an operator $\mathcal{O}$ on $\mathfrak{F}_{0}$ can be conveniently expressed in terms of the coherent states [57]:

$$
\operatorname{Tr}_{\mathfrak{F}_{0}} \mathcal{O}=\prod_{n=1}^{\infty} \int d \bar{\xi}_{n}^{\prime} d \xi_{n} e^{-\left\langle\bar{\xi}_{n}^{\prime}, \xi_{n}\right\rangle}\left\langle e^{\frac{1}{\sqrt{n}} \xi_{n}^{\prime}(-n)} \mathbf{1} \mid \mathcal{O} e^{\frac{1}{\sqrt{n}} \xi_{n}(-n)} \mathbf{1}\right\rangle
$$

It follows from this representation that

Proposition 6.38. For $0<|q|<|y|<1$, we have

$$
\operatorname{Tr}_{\mathfrak{F}_{0}} V(-\alpha, 1) V(\alpha, y) q^{L(0)-\frac{\ell}{24}}=\frac{1}{\eta(\tau)^{\ell} E(\tau, \nu)^{\|\alpha\|^{2}}} .
$$


Proof. Using (6.21) the left hand side can be rewritten as

$$
q^{-\frac{\ell}{24}} \prod_{n=1}^{\infty} \int d \overline{\xi_{n}^{\prime}} d \xi_{n} e^{-\left\langle\bar{\xi}_{n}^{\bar{y}}, \xi_{n}\right\rangle}\left\langle e^{\frac{1}{\sqrt{n}} \xi_{n}^{\prime}(-n)} \mathbf{1} \mid V(-\alpha, 1) V(\alpha, y) e^{\frac{q^{n}}{\sqrt{n}} \xi_{n}(-n)} \mathbf{1}\right\rangle .
$$

Then the integrand of each factor becomes

$$
\begin{aligned}
& \frac{\exp \left[-\left\langle\overline{\xi_{n}^{\prime}}, \xi_{n}\right\rangle\right]}{\left(y^{-1 / 2}-y^{1 / 2}\right)^{\|\alpha\|^{2}}}\left\langle e^{\frac{1}{\sqrt{n}} \xi_{n}^{\prime}(-n)} \mathbf{1} \mid e^{\frac{1-y^{n}}{\sqrt{n}}\left\langle\alpha, \overline{\xi_{n}^{\prime}}\right\rangle} e^{\frac{\left(1-y^{-n}\right) q^{n}}{\sqrt{n}}\left\langle\alpha, \xi_{n}\right\rangle} e^{\frac{q^{n}}{\sqrt{n}} \xi_{n}(-n)} \mathbf{1}\right\rangle \\
& =\frac{1}{\left(y^{-1 / 2}-y^{1 / 2}\right)^{\|\alpha\|^{2}}} \exp \left[-\left(1-q^{n}\right)\left\langle\overline{\xi_{n}^{\prime}}, \xi_{n}\right\rangle\right. \\
& \left.+\frac{\left(1-y^{-n}\right) q^{n}}{\sqrt{n}}\left\langle\alpha, \xi_{n}\right\rangle+\frac{1-y^{n}}{\sqrt{n}}\left\langle\alpha, \bar{\xi}_{n}^{\prime}\right\rangle\right],
\end{aligned}
$$

where we used (6.27). By performing the integrals using Lemma 6.35 we thus obtain

$$
\frac{1}{\eta(\tau)^{\ell}\left(y^{-1 / 2}-y^{1 / 2}\right)^{\|\alpha\|^{2}}} \exp \left[\|\alpha\|^{2} \sum_{n=1}^{\infty} \frac{\left(2-y^{n}-y^{-n}\right) q^{n}}{n\left(1-q^{n}\right)}\right],
$$

which can be cast in the desired form thanks to the identity

$$
\prod_{n=1}^{\infty} \frac{1}{1-t q^{n-1}}=\exp \left[\sum_{n=1}^{\infty} \frac{t^{n}}{n\left(1-q^{n}\right)}\right] \text {. }
$$

Suppose that we are in the situation (6.29). We set $\alpha=c_{1}\left(\mathcal{O}_{X}\left(C_{0}\right)\right)$ so that $\|\alpha\|^{2}=-C_{0}^{2}=2$. Since $\ell=24$, we see that the right hand side of $(6.39)$ reduces to $1 / \chi_{10,1}(\tau, \nu)$.

Let $\mathcal{N}$ be defined by

$$
L(0)=\frac{1}{2} \sum_{i=1}^{\ell} e^{i}(0) e_{i}(0)+\mathcal{N} .
$$

Consider the spectral decomposition $\mathcal{N}=\sum_{d=0}^{\infty} d \mathrm{P}_{d}$ where $\mathrm{P}_{d}$ is the projection operator onto the eigensubspace with eigenvalue $d$ of $\mathfrak{F}_{0}$ with the obvious properties: $\mathrm{P}_{d}^{2}=\mathrm{P}_{d}, \mathrm{P}_{d} \mathrm{P}_{s}=0$ if $d \neq s$, and $\sum_{d=0}^{\infty} \mathrm{P}_{d}=i d$. Then we find that 
Lemma 6.45. For $0<|q|<|y|<1$,

$$
\begin{aligned}
& \operatorname{Tr}_{\mathfrak{F}_{0}} V(-\alpha, 1) V(\alpha, y) q^{L(0)-\frac{\ell}{24}} \\
& =\sum_{h=0}^{\infty} \sum_{d=0}^{\infty} q^{h-\frac{\ell}{24}} y^{d+\frac{\|\alpha\|^{2}}{2}-h} \operatorname{Tr}_{\mathfrak{F}_{0}} V(-\alpha, 1) \mathrm{P}_{d} V(\alpha, 1) \mathrm{P}_{h} .
\end{aligned}
$$

Proof. Using (6.28) we see that the left hand side is equal to

$$
\begin{aligned}
& \operatorname{Tr}_{\mathfrak{F}_{0}} V(-\alpha, 1) y^{L(0)} V(\alpha, 1) y^{-L(0)} q^{L(0)-\frac{\ell}{24}} \\
& \quad=\operatorname{Tr}_{\mathfrak{F}_{0}} V(-\alpha, 1) y^{L(0)} \sum_{d=0}^{\infty} \mathrm{P}_{d} V(\alpha, 1) y^{-L(0)} q^{L(0)-\frac{\ell}{24}} \sum_{h=0}^{\infty} \mathrm{P}_{h} \\
& \quad=\sum_{h=0}^{\infty} \sum_{d=0}^{\infty} \operatorname{Tr}_{\mathfrak{F}_{0}} V(-\alpha, 1) y^{\frac{\|\alpha\|^{2}}{2}}+d \mathrm{P}_{d} V(\alpha, 1) y^{-h} q^{h-\frac{\ell}{24}} \mathrm{P}_{h}
\end{aligned}
$$

An immediate consequence of Lemma 6.45 is the following claim equivalent to Theorem 5.80:

Proposition 6.48. With the identification (6.29) and $\alpha=c_{1}\left(\mathcal{O}_{X}\left(C_{0}\right)\right)$, there exists a relation

$$
\chi\left(\mathcal{C}_{h}^{[d]}\right)=\operatorname{Tr}_{\mathfrak{F}_{0}} V(-\alpha, 1) \mathrm{P}_{d} V(\alpha, 1) \mathrm{P}_{h},
$$

for each pair $(h, d)$ of nonnegative integers.

Remark 6.50. With this expression at hand the degree-genus duality (5.86) follows immediately from the cyclic symmetry of the trace and the fact that the right hand side of (6.49) is invariant under the exchange $\alpha \leftrightarrow-\alpha$.

\subsection{Two-point correlation functions and elliptic genus}

We wish to take this opportunity to make the observation in [64] more explicit. This subsection is not logically related to the main theme of this paper and may be skipped. 
Let us recall the definition of the Weierstraß $\sigma$ function:

$$
\sigma(\tau, \nu)=-2 \pi \sqrt{-1} \nu \prod_{\substack{\omega \in \mathbb{Z}+\mathbb{Z} \tau \\ \omega \neq 0}}\left(1-\frac{\nu}{\omega}\right) \exp \left[\frac{\nu}{\omega}+\frac{1}{2}\left(\frac{\nu}{\omega}\right)^{2}\right] .
$$

As is well-known this is related to the Weierstraß $\wp$-function

$$
\wp(\tau, \nu):=\frac{1}{(2 \pi \sqrt{-1})^{2}}\left\{\frac{1}{\nu^{2}}+\sum_{\substack{\omega \in \mathbb{Z}+\mathbb{Z} \tau \\ \omega \neq 0}}\left(\frac{1}{(\nu-\omega)^{2}}-\frac{1}{\omega^{2}}\right)\right\},
$$

by the relation

$$
\wp(\tau, \nu)=-\left(y \frac{\partial}{\partial y}\right)^{2} \log \sigma(\tau, \nu)
$$

The $\sigma$ function is related to the prime form by $^{7}$

$$
\begin{aligned}
\sigma(\tau, \nu) & =\exp \left(x^{2} E_{2}(\tau) / 24\right) E(\tau, \nu) \\
& =-\sqrt{-1} x \exp \left(\sum_{k=2}^{\infty} \frac{(-1)^{k} B_{2 k}}{2 k(2 k) !} x^{2 k} E_{2 k}(\tau)\right) .
\end{aligned}
$$

In analogy to $\wp(\tau, \nu)$ let us introduce

$$
\Gamma(\tau, \nu):=-\left(y \frac{\partial}{\partial y}\right)^{2} \log E(\tau, \nu) .
$$

Then apparently we have a relation:

$$
\wp(\tau, \nu)=\Gamma(\tau, \nu)+\frac{1}{12} E_{2}(\tau)
$$

Note that while $\wp(\tau, \nu)$ is a (meromorphic) Jacobi form of weight 2 and index $1, \Gamma(\tau, \nu)$ is not. Explicitly one finds that

$$
\begin{aligned}
\Gamma(\tau, \nu) & =\frac{1}{\left(y^{-1 / 2}-y^{1 / 2}\right)^{2}}-y \frac{\partial}{\partial y} \sum_{n=1}^{\infty}\left(\frac{-y q^{n}}{1-y q^{n}}+\frac{y^{-1} q^{n}}{1-y^{-1} q^{n}}\right) \\
& =\frac{1}{\left(y^{-1 / 2}-y^{1 / 2}\right)^{2}}+y \frac{\partial}{\partial y} \sum_{n=1}^{\infty} \sum_{k=1}^{\infty}\left(y^{k}-y^{-k}\right) q^{n k} \\
& =\frac{1}{\left(y^{-1 / 2}-y^{1 / 2}\right)^{2}}+\sum_{n=1}^{\infty} \frac{n\left(y^{n}+y^{-n}\right) q^{n}}{1-q^{n}} .
\end{aligned}
$$

${ }^{7}$ In the traditional theory of elliptic functions, $E_{2}$ is usually denoted as $\eta_{1}$ up to a scalar multiplication. 
For any $b \in \mathcal{V}$ and $\beta \in \Lambda$ such that $\langle b, \beta\rangle=0$, define

$$
U_{t}(\beta, b, y)=V(\beta, y) e^{t P_{-}(b, y)} e^{t P_{+}(b, y)},
$$

where $t$ is a formal variable. Observe that

$$
\left.\frac{d}{d t} U_{t}(\beta, b, y)\right|_{t=0}=P(b, y) V(\beta, y)=: W(\beta, b, y),
$$

where $P(b, y):=P_{+}(b, y)+P_{-}(b, y)$.

Proposition 6.60. Suppose that $a, b \in \mathcal{V}$ and $\beta \in \Lambda$ satisfy $\langle a, \beta\rangle=$ $\langle b, \beta\rangle=0$. Then, for $0<|q|<|y|<1$, we obtain that

$$
\begin{aligned}
\operatorname{Tr}_{\mathfrak{F}_{0}} & U_{t}(-\beta, a, 1) U_{s}(\beta, b, y) q^{L(0)-\frac{\ell}{24}}=\frac{1}{\eta(\tau)^{\ell} E(\tau, \nu)^{\|\beta\|^{2}}} \\
& \times \exp \left(t s\langle a, b\rangle \Gamma(\tau, \nu)+\frac{1}{24}\left(t^{2}\|a\|^{2}+s^{2}\|b\|^{2}\right)\left(1-E_{2}(\tau)\right)\right) .
\end{aligned}
$$

Proof. The calculation is similar to that in the proof of Proposition 6.38. The left hand side is equal to

$$
\begin{gathered}
q^{-\frac{\ell}{24}} \prod_{n=1}^{\infty} \int d \bar{\xi}_{n}^{\prime} d \xi_{n} \frac{1}{\left(y^{-1 / 2}-y^{1 / 2}\right)^{\|\beta\|^{2}}} \exp \left[\frac{t s\langle a, b\rangle}{\left(y^{-1 / 2}-y^{1 / 2}\right)^{2}}\right] \\
\times \exp \left[-\left(1-q^{n}\right)\left\langle\bar{\xi}_{n}^{\prime}, \xi_{n}\right\rangle+q^{n}\left\langle\frac{\left(1-y^{-n}\right)}{\sqrt{n}} \beta+\sqrt{n}\left(t a+s y^{-n} b\right), \xi_{n}\right\rangle\right. \\
\left.+\left\langle\frac{1-y^{n}}{\sqrt{n}} \beta+\sqrt{n}\left(t a+s y^{n} b\right), \overline{\xi_{n}^{\prime}}\right\rangle\right] .
\end{gathered}
$$

By performing the Gaussian integrals we obtain that

$$
\begin{aligned}
& \frac{1}{\eta(\tau)^{\ell} E(\tau, \nu)^{\|\beta\|^{2}}} \exp \left[t s\langle a, b\rangle\left(\frac{1}{\left(y^{-1 / 2}-y^{1 / 2}\right)^{2}}+\sum_{n=1}^{\infty} \frac{n\left(y^{n}+y^{-n}\right) q^{n}}{1-q^{n}}\right)\right. \\
& \left.+\left(t^{2}\|a\|^{2}+s^{2}\|b\|^{2}\right) \sum_{n=1}^{\infty} \frac{n q^{n}}{1-q^{n}}\right] .
\end{aligned}
$$

This readily leads to the desired result.

Suppose again that $X$ is a $K 3$ surface but set $\Lambda=H^{2 *}(X, \mathbb{Z})(-1) \oplus$ $H(-1)$ so that $\ell=26$. Assume that $H(-1)$ is generated by $\alpha$ and 
$\beta$ where $\|\alpha\|^{2}=2,\|\beta\|^{2}=0$ and $\langle\alpha, \beta\rangle=-1$. Let $\left\{f_{i}\right\}$ be a basis of $H^{2 *}(X, \mathbb{Z})(-1)$ and let $\left\{f^{i}\right\}$ be the dual basis. Then (6.59) and Proposition 6.60 show that

$$
\frac{\eta(\tau)^{2} \sum_{i=1}^{24} \operatorname{Tr}_{\mathfrak{F}_{0}} W\left(-\beta, f^{i}, 1\right) W\left(\beta, f_{i}, y\right) q^{L(0)-26 / 24}}{\left.\eta(\tau)^{2} \operatorname{Tr}_{\mathfrak{F}_{0}} V(-\alpha, 1) V(\alpha, y)\right) q^{L(0)-26 / 24}}=24 \Gamma(\tau, \nu) E(\tau, \nu)^{2} .
$$

The left hand side is the ratio of two-point functions of vector particles and tachyons if we make an analogy with bosonic open string. (The expression $\eta(\tau)^{2}$ stems from the ghost sector.) If we replace $\Gamma(\tau, \nu)$ by the Jacobi form $\wp(\tau, \nu)$ one obtains the elliptic genus of $X$ in the form presented in [64]:

$$
\mathcal{E}_{X}(\tau, \nu)=24 \wp(\tau, \nu) E(\tau, \nu)^{2} .
$$

Remark 6.66. If $X$ is an elliptic $K 3$ surface with a section $\sigma$ and a fiber $f$, we may instead set $\alpha=c_{1}\left(\mathcal{O}_{X}(\sigma)\right)$ and $\beta=c_{1}\left(\mathcal{O}_{X}(f)\right)$. Then $\left\{f_{i}\right\}$ must be a basis of $(\mathbb{Z} \alpha+\mathbb{Z} \beta)^{\perp}$.

\section{A conifold and Chern-Simons theory}

As a simple application of the infinite product representation of the string partition function, we now reproduce some earlier obtained results on the relation between topological type IIA string near a conifold point and the $S U(\infty)$ Chern-Simons theory on a 3-dimensional sphere $S^{3}[41-43,58,94,104]$.

Let us set $\xi=q_{2}=p q^{-1}$. Then it is expected that the limit $\log \xi \rightarrow$ 0 corresponds to the point where a conifold singularity arises. We first set $z=0$, then in the neighborhood of this limit there is a factor

$$
\prod_{j=-\infty}^{\infty}\left(1-\xi y^{j}\right)^{\frac{|j|}{2}}=\exp \left(\sum_{g=0}^{\infty} x^{2 g-2} m_{g} \mathrm{Li}_{3-2 g}(\xi)\right),
$$

raised to the power of $c_{0}(-1,0)=-2$ in the infinite product (4.16). Here we used (4.13). Intuitively this factor corresponds to the bound states of a $D 2$-brane and $D 0$-branes (with the charge conjugation symmetry imposed) where the $D 2$-brane wraps once around the shrinking $\mathbb{P}^{1}$ with $\log \xi$ being its complexified Kähler parameter. 
According to the fundamental work [101], up to the framing ambiguity the partition function of the Chern-Simons theory on $S^{3}$ with gauge group $G$ and a positive integer coupling $k$ is equal to $S_{k \Lambda_{0}, k \Lambda_{0}}$ where $S_{k \Lambda_{0}, k \Lambda_{0}}$ is one of the entries of the transformation matrix of the level $k$ Weyl-Kac characters of the affine Lie algebra of $G$ under the modular transformation $\tau \rightarrow-\frac{1}{\tau}$. We recall that $S_{k \Lambda_{0}, k \Lambda_{0}}$ is expressed by the classical Weyl denominator of $G$ [59]. In the case of $G=S U(N)$, the partition function $Z_{W}\left(S^{3} ; N, k\right)$ can be explicitly written down [17] as

$$
Z_{W}\left(S^{3} ; N, k\right)=\left(\frac{N}{N^{\prime N-1}}\right)^{-\frac{1}{2}} \prod_{j=1}^{N-1}\left(2 \sin \frac{\pi j}{N^{\prime}}\right)^{N-j}
$$

where $N^{\prime}=k+N$. It is well-known that there exists level-rank duality:

$$
\sqrt{N} Z_{W}\left(S^{3} ; N, k\right)=\sqrt{k} Z_{W}\left(S^{3} ; k, N\right)
$$

from which it follows that

$$
\begin{aligned}
& Z_{W}\left(S^{3} ; N, k\right) \\
& =\sqrt{\frac{N^{\prime}}{N}} N^{\prime-\frac{N^{\prime}}{4}} \mathbf{e}\left[\rho_{N^{\prime}, N}\right] \prod_{j=1}^{N^{\prime}-N-1}\left(1-\mathbf{e}\left[\frac{N+j}{N^{\prime}}\right]\right)^{\frac{j}{2}} \prod_{j=1}^{N-1}\left(1-\mathbf{e}\left[\frac{N-j}{N^{\prime}}\right]\right)^{\frac{j}{2}}
\end{aligned}
$$

where we have set

$$
\begin{aligned}
\rho_{N^{\prime}, N}=\{ & \left.-\frac{1}{12}\left(N / N^{\prime}\right)^{3}+\frac{1}{8}\left(N / N^{\prime}\right)^{2}-\frac{1}{48}\right\} N^{\prime 2} \\
& +\left\{-\frac{1}{8}\left(N / N^{\prime}\right)+\frac{1}{16}\right\} N^{\prime}+\frac{1}{12}\left(N / N^{\prime}\right)-\frac{1}{24} .
\end{aligned}
$$

We make the identification: $\xi=\mathbf{e}\left[N / N^{\prime}\right]$ and $y=\mathbf{e}\left[1 / N^{\prime}\right]$. This is a familiar choice of variables when we relate the HOMFLY polynomial of knot theory to the $S U(N)$ Chern-Simons theory. Then we discern the infinite product (7.1) when $N^{\prime}>N>>1$. Note however that since the Chern-Simons theory is an open string theory, the symmetry under $y \leftrightarrow y^{-1}$, which is peculiar to a closed string theory, is violated in the whole expression (7.4). 
Using the formulas in Appendix A, we have ${ }^{8}$

$$
\begin{aligned}
m_{0} \mathcal{L i}_{3}(\xi)= & -\frac{(\log \xi)^{2}}{2} \log (\log \xi)+\zeta(3) \\
& -\frac{(\log \xi)^{3}}{12}+\sum_{k=2}^{\infty} \frac{\zeta(3-2 k)}{(2 k) !}(\log \xi)^{2 k} \\
m_{1} \mathcal{L i}_{1}(\xi)= & -\frac{1}{12} \log (\log \xi)-\frac{1}{24} \log \xi+\sum_{k=1}^{\infty} \frac{\zeta(1-2 k)}{12(2 k) !}(\log \xi)^{2 k}
\end{aligned}
$$

and for $g \geq 2$

$$
\begin{aligned}
m_{g} \operatorname{Li}_{3-2 g}(\xi) & =\frac{m_{g}(2 g-3) !}{(\log \xi)^{2 g-2}}+\sum_{k=0}^{\infty} \frac{m_{g} \zeta(3-2 g-2 k)}{(2 k) !}(\log \xi)^{2 k} \\
& =\frac{(-1)^{g-1} \chi_{g, 0}}{(\log \xi)^{2 g-2}}+\sum_{k=0}^{\infty} \frac{(-1)^{k} 2 \zeta(2 g-2+2 k)}{(2 \pi)^{2 g-2+2 k}} \chi_{g, 2 k}(\log \xi)^{2 k}
\end{aligned}
$$

These directly reproduce the behaviors of the Gromov-Witten potentials in the vicinity of a conifold which were discussed in $[41,58,94]$ and especially in $[42,43]$.

\section{Discussions}

In this paper we have argued that the string partition functions of certain elliptically and $K 3$ fibered Calabi-Yau 3-folds in a particular limit should have the infinite product representation (4.16). We have used the lifting procedure of Jacobi forms in an essential way. It was rather ironic and somewhat against the initial impression that purely from the viewpoint of lifting, the amount of difficulty in computing the Gromov-Witten potential decreases as the genus $g$ increases; in fact there is no contribution to the "Weyl vector" when $g>1$.

Although we cannot be too optimistic since the lifting procedure of Jacobi forms should be useful only for the fibered Calabi-Yau 3folds, it is hard to resist the temptation to make a bolder conjecture:

\footnotetext{
${ }^{8}$ For simplicity, we use $\mathcal{L} \mathrm{i}_{r}$ instead of $\mathrm{Li}_{r}$ when $r>0$.
} 
For a general Calabi-Yau 3-fold the string partition function may be expressed in a form that schematically looks like:

$$
\mathcal{Z}=\exp \left(x^{-2} F_{0}^{(0)}+F_{1}^{(0)}\right) \prod_{\left(\left\{n_{i}\right\}, j\right)>0}\left(1-\prod_{i=1}^{l} q_{i}^{n_{i}} y^{j}\right)^{D\left(\left\{n_{i}\right\}, j\right)}
$$

The major portion of this paper has been devoted to an interpretation by $D 2-D 0$ bound states. It is obvious that one of challenging but interesting directions for further research is to place the study of $D 2-D 0$ bound states on a mathematically rigorous footing for general Calabi-Yau 3-folds and ask if the Gromov-Witten theory can be totally reformulated in that picture. This, if achieved, may shed some light on the (homological) mirror conjecture. We have suggested in this work that an appropriate language toward this goal may be that of coherent systems of dimension 1 . Given our success in the $K 3$ case, this approach should merit a close scrutiny.

Also it would be most desirable to find out, if any, an organizing theory whose partition function is directly given by (4.16) or (8.1). The theory will presumably have some flavor of Chern-Simons theory. Since the infinite product representations (4.16) or (8.1) have strong resemblance to the Weyl-Kac-Borcherds denominator, it seems natural to expect the existence of some nice algebra of $D 0$-, $D 2$-branes. It should be emphasized that, while the Borcherds denominators are generally expected to be related to enumeration problems of curves or $D 2$-branes on surfaces, in the situation of this paper where fibered Calabi-Yau 3 -folds are relevant, the analogy to the Weyl-Kac-Borcherds denominator was most evident only after we incorporate $D 0$-branes in addition to $D 2$-branes. In this analogy, the Euler characteristics of the moduli spaces of coherent systems must in an appropriate sense be interpreted as "root multiplicities". Some aspects of the algebra of $D$-branes were studied in [50]. Identifying the algebra should help in knowing the (necessarily infinite-dimensional) gauge symmetry of the organizing theory.

Another remaining issue, which we were unable to address in this work, is to investigate the automorphic properties of the infinite product which we used for the string partition function. 


\section{Appendix A}

We define the Bernoulli numbers $B_{n}(n=0,1,2, \ldots)$ by

$$
\frac{t}{e^{t}-1}=\sum_{n=0}^{\infty} B_{n} \frac{t^{n}}{n !} \text {. }
$$

Hence we have

$$
B_{0}=1, \quad B_{1}=-\frac{1}{2}, \quad B_{2}=\frac{1}{6}, \quad B_{4}=-\frac{1}{30}, \ldots
$$

and $B_{2 k+1}=0(k \geq 1)$. The values of the Riemann zeta function at integers can sometimes be expressed in terms of the Bernoulli numbers:

$$
\begin{aligned}
\zeta(2 k) & =\frac{(2 \pi)^{2 k}}{2(2 k) !}\left|B_{2 k}\right|, \quad(k \geq 0), \\
\zeta(1-2 k) & =-\frac{B_{2 k}}{2 k}, \quad(k \geq 1) .
\end{aligned}
$$

The series

$$
\Omega(\xi, s)=\sum_{n=1}^{\infty} \frac{\xi^{n}}{n^{s}}, \quad(\operatorname{Re} s>1,|\xi|<1),
$$

and its analytic continuation frequently appeared in the past. See for instance [96] [29]. When $s=r \in \mathbb{Z}$ we will set

$$
\operatorname{Li}_{r}(\xi)=\Omega(\xi, r) .
$$

As the notation suggests, $\operatorname{Li}_{r}(\xi)$ is the usual polylogarithm when $r>0$. On the other hand, if $r \leq 0, \mathrm{Li}_{r}(\xi)$ is a rational function. The following differential-difference equation is well-known:

$$
\xi \frac{\partial}{\partial \xi} \operatorname{Li}_{r}(\xi)=\operatorname{Li}_{r-1}(\xi)
$$

For instance, we have

$$
\begin{aligned}
\operatorname{Li}_{1}(\xi) & =-\log (1-\xi) \\
\operatorname{Li}_{0}(\xi) & =\frac{\xi}{1-\xi} \\
\operatorname{Li}_{-1}(\xi) & =\frac{\xi}{(1-\xi)^{2}}
\end{aligned}
$$


If $r>0$, the polylogarithm $\mathrm{Li}_{r}(\xi)$ can be analytically continued to a multi-valued holomorphic function on $\mathbb{P}^{1} \backslash\{0,1, \infty\}$. As in $[9,65]$ we introduce $\mathcal{L} \mathrm{i}_{r}(\xi)$ as $\operatorname{Li}_{r}(\xi)$ modulo any $\mathbb{Q}$-linear combinations of $S_{r-1}(\xi), S_{r-2}(\xi), \ldots, S_{0}(\xi)$ where $S_{r-j}(\xi):=\frac{(2 \pi \sqrt{-1})^{j}}{(r-j) !}(\log \xi)^{r-j}, \quad(1 \leq$ $j \leq r)$. This is to kill off the monodromy of $\operatorname{Li}_{r}(\xi)$ and attain the effective single-valuedness.

When $r$ is a positive integer, we have the expansion [96] [29]

$$
\mathrm{Li}_{r}(\xi)=\frac{(\log \xi)^{r-1}}{(r-1) !}[\psi(r)-\psi(1)-\log (-\log \xi)]+\sum_{j=0}^{\infty} \frac{\zeta(r-j)}{j !}(\log \xi)^{j}
$$

where ' stands for the omission of the case $j=r-1$ and $\psi(t)=$ $\frac{d}{d t} \log \Gamma(t)$. Note that $\psi(r)-\psi(1)=\sum_{k=1}^{r-1} \frac{1}{k}$ when $r$ is an integer greater than 1 . This expansion can be simplified for $\mathcal{L} \mathrm{i}_{r}(\xi)$ as

$$
\mathcal{L} \mathrm{i}_{r}(\xi)=-\frac{(\log \xi)^{r-1}}{(r-1) !} \log (\log \xi)+\sum_{j=0}^{\infty} \frac{\zeta(r-j)}{j !}(\log \xi)^{j}
$$

where " stands for the omissions of the case $j=r-1$ as well as the cases where the summand can be expressed as $\mathbb{Q}$-linear combinations of $S_{r-1}(\xi), S_{r-2}(\xi), \ldots, S_{0}(\xi)$.

If instead $r$ is 0 or a negative integer, we have [96] [29]

$$
\operatorname{Li}_{r}(\xi)=\frac{|r| !}{(-\log \xi)^{|r|+1}}-\sum_{j=0}^{\infty} \frac{B_{|r|+j+1}}{(|r|+j+1) j !}(\log \xi)^{j}
$$

We note that the expansion (2.12) can be obtained from this by setting $r=-1$.

\section{Appendix B}

Let $A$ be an abelian surface over $\mathbb{C}$. Set $A^{[\ell]}:=$ Hilb $_{A}^{\ell}$. Let $\kappa_{\ell}: A^{[\ell]} \rightarrow A$ be the morphism obtained by composing the Hilbert-Chow morphism $\pi_{\ell}: A^{[\ell]} \rightarrow A^{(\ell)}$ and the sum map $\sigma_{\ell}: A^{(\ell)} \rightarrow A$. Beauville [4] showed that $A^{\langle\ell-1\rangle}:=\kappa_{\ell}^{-1}(0)$ is an irreducible symplectic manifold of dimension $2 \ell-2$. In particular $A^{\langle 1\rangle}$ is the Kummer surface. 
Let $\tilde{\phi}_{-2,1}(\tau, \nu)$ be the weak Jacobi form of weight -2 and index 1 introduced in [27]. We have a relation $\tilde{\phi}_{-2,1}(\tau, \nu)=E(\tau, \nu)^{2}$.

Conjecture B.1. The elliptic genus of $A^{\langle\ell-1\rangle}$ is given by

$$
\mathcal{E}_{A^{\langle\ell-1\rangle}}(\tau, \nu)=\ell^{4} \frac{\left.\tilde{\phi}_{-2,1}\right|_{V_{\ell}}(\tau, \nu)}{\tilde{\phi}_{-2,1}(\tau, \nu)} .
$$

Some evidence for this conjecture is as follows. First, the elliptic genus $\mathcal{E}_{A^{\langle\ell-1\rangle}}(\tau, \nu)$ must be a weak Jacobi form of weight 0 and index $\ell-1$ since $c_{1}\left(A^{\langle\ell-1\rangle}\right)=0$ [66]. It is easy to see that the right hand side of (B.2) has this property. Next, one can check the conjecture at the level of $\chi_{y}$ genus: Suppose that the conjecture holds. Then by noting $\tilde{\phi}_{-2,1}(\tau, \nu)=(1-y)^{2} / y+\ldots$, we must have

$$
\begin{aligned}
\chi_{y}\left(A^{\langle\ell-1\rangle}\right) & =\left.y^{\ell-1} \ell^{4} \frac{\left.\tilde{\phi}_{-2,1}\right|_{V_{\ell}}(\tau, \nu)}{\tilde{\phi}_{-2,1}(\tau, \nu)}\right|_{q^{0}} \\
& =\left.\frac{y^{\ell-1}}{\tilde{\phi}_{-2,1}(\tau, \nu)} \ell \sum_{\substack{a d=\ell \\
a>0}} d^{2} \sum_{b=0}^{d-1} \tilde{\phi}_{-2,1}\left(\frac{a \tau+b}{d}, a \nu\right)\right|_{q^{0}} \\
& =y^{\ell-1} \ell \sum_{d \mid \ell} d^{3} \frac{\left(1-y^{\ell / d}\right)^{2} / y^{\ell / d}}{(1-y)^{2} / y} \\
& =\ell \sum_{d \mid \ell} d^{3}\left(1+y+\cdots+y^{\ell / d-1}\right)^{2} y^{\ell-\ell / d} .
\end{aligned}
$$

However, the last expression has already appeared in $[38,39]$.

Remark B.4. The Hilbert schemes $X^{[d]}$ of a projective $K 3$ surface $X$ and the higher order Kummer varieties $A^{\langle\ell-1\rangle}$ are two fundamental series of irreducible symplectic manifolds [4]. If the conjectures are true, the elliptic genera of $X^{[d]}$ and $A^{\langle\ell-1\rangle}$ can be expressed respectively in terms of $\tilde{\phi}_{0,1}(\tau, \nu)$ and $\tilde{\phi}_{-2,1}(\tau, \nu)$ by using the Hecke operators. Here $\tilde{\phi}_{0,1}(\tau, \nu)$ and $\tilde{\phi}_{-2,1}(\tau, \nu)$ are known [27] to be the generators of the ring of weak Jacobi forms of even weight, thus they are equally fundamental in the theory of Jacobi forms. 


\section{References}

[1] A.B. Altman and S.L. Kleiman, Compactifying the Picard scheme, Adv. in Math., 35 (1980), 50-112.

[2] P.S. Aspinwall and D. Morrison, Topological field theory and rational curves, Commun. Math. Phys., 151 (1993), 245-262, hep-th/9110048.

[3] V. Baranovsky, Moduli of sheaves on surfaces and action of the oscillator algebra, math/9811092.

[4] A. Beauville, Variétés kähleriennes dont la première classe de Chern est nulle, J. Diff. Geom., 18 (1983), 755-782.

[5] A. Beauville, Counting rational curves on K3 surfaces, Duke Math. J., 97 (1999), 99-108, alg-geom/9701019.

[6] K. Behrend, Gromov-Witten invariants in algebraic geometry, Invent. Math., 127 (1997), 601-617, alg-geom/9601011.

[7] K. Behrend and B. Fantechi, The intrinsic normal cone, Invent. Math., 128 (1997), 45-88, alg-geom/9601010.

[8] K. Behrend and Yu. Manin, Stacks of stable maps and Gromov-Witten invariants, Duke. J. Math., 85 (1996), 1-60, alg-geom/9506023.

[9] A. Beilinson and A. Levin, The Elliptic polylogarithm, in 'Motives', (U. Jannsen, S. Kleiman, J.-P. Serre, eds.), Proc. Symp. Pure Math., 55, Amer. Math. Soc., 1994, Part 2, 123-190.

[10] M. Bershadsky, S. Cecotti, H. Ooguri, and C. Vafa, Holomorphic anomalies in topological field theories, (with an appendix by S. Katz), Nucl. Phys., B405 (1993), 279-304, hep-th/9302103; Kodaira-Spencer theory of gravity and exact results for quantum string amplitudes, Commun. Math. Phys., 165 (1994), 311-427, hep-th/9309140.

[11] M. Bershadsky, A. Johansen, V. Sadov, and C. Vafa, Topological reduction of $4 D S Y M$ to $2 D$ o-models, Nucl. Phys., B448 (1995), 166, hep-th/9501096. 
[12] R.E. Borcherds, Automorphic forms on $\mathrm{O}_{s+2,2}(\mathbf{R})$ and infinite products, Invent. Math., 120 (1995), 161-213.

[13] R.E. Borcherds, Automorphic forms with singularities on Grassmannians, Invent. Math., 132 (1998), 491-562, alg-geom/9609022.

[14] S. Bradlow, Vortices in holomorphic line bundles over closed Kähler manifolds, Comm. Math. Phys., 135 (1990), 1-17.

[15] S. Bradlow, G.D. Daskalopoulos, O. García-Prada, R. Wentworth, Stable augmented bundles over Riemann surfaces, in 'Vector bundles in algebraic geometry' (Durham, 1993), (N.J. Hitchin, P.E. Newstead and W.M. Oxbury, eds), London Math. Soc. Lecture Note Ser., 208, Cambridge Univ. Press, 1995.

[16] G.E. Bredon, Sheaf theory, 2nd edition, Graduate Texts in Mathematics, 170, Springer-Verlag, 1997.

[17] M. Camperi, F. Levstein, and G. Zemba, The large $N$ limit of Chern-Simons gauge theory, Phys. Lett., B247 (1990), 549-554.

[18] J. Cheeger, Analytic torsion and the heat equation, Ann. of Math., 109 (1979), 259-300.

[19] J. Cheah, On the cohomology of Hilbert schemes of points, J. Alg. Geom., 5 (1996), 479-511.

[20] N. Chriss and V. Ginzburg, Representation Theory and Complex Geometry, Birkhäuser, 1997.

[21] H.R. Christiansen, C. Núñez and F.A. Schaposnik, Uniqueness of Bogomol'nyi equations and Born-Infeld like supersymmetric theories, Phys. Lett., B441 (1998), 185-190, hep-th/9807197.

[22] P. Deligne, Théorie de Hodge III, Inst. Hautes Etudes Sci. Publ. Math., 44 (1974), 5-77.

[23] R. Dijkgraaf, Mirror symmetry and elliptic curves, in The moduli space of curves, (R. Dijkgraaf, C. Faber and G. van der Geer, eds.), Progr. Math., 129, Birkhäuser, 1995. 
[24] R. Dijkgraaf, G. Moore, E. Verlinde, and H. Verlinde, Elliptic genera of symmetric products and second quantized strings, Commun. Math. Phys., 185 (1997), 197-209.

[25] L.J. Dixon, V. Kaplunovsky, and J. Louis, Moduli dependence of string loop corrections to gauge coupling constants, Nucl. Phys. B355 (1991) 649-688.

[26] J. Edelstein, C. Núñez, and F.A. Schaposnik, Supersymmetry and Bogomol'nyi equations in the Abelian Higgs Model, Phys. Lett., B329 (1994), 39-45, hep-th/9311055.

[27] M. Eichler and D. Zagier, The Theory of Jacobi Forms, Progress in Mathematics 55, Birkhäuser, 1985.

[28] G. Ellingsrud, L. Göttsche, M. Lehn, On the cobordism class of the Hilbert scheme of a surface, math/9904095.

[29] A. Erdelyi, W. Magnus, F. Oberhettinger, and F.G. Tricomi, Higher transcendental functions, Vol. 1, McGraw-Hill, 1953.

[30] C. Faber, A conjectural description of the tautological ring of the moduli space of curves, preprint, 1996.

[31] C. Faber and R. Pandharipande, Hodge integrals and GromovWitten theory, math.AG/9810173.

[32] B. Fantechi, L. Göttsche, D. van Straten, Euler number of the compactified Jacobian and multiplicity of rational curves, J. Alg. Geom., 8 (1999), 115-133, alg-geom/9708012.

[33] R. Fintushel and R.J. Stern, Knots, links, and 4-manifolds, Invent. Math., 134 (1998), 363-400, dg-ga/9612014.

[34] W. Fulton, Intersection Theory, 2nd edition, Springer-Verlag, 1998.

[35] R. Friedman, Algebraic surfaces and holomorphic vector bundles, Springer-Verlag, 1998.

[36] O. García-Prada, Seiberg-Witten invariants and vortex equations, in 'Symétries quantiques' (A. Connes, K. Gawedzki and J. ZinnJustin, eds), North-Holland, 1998. 
[37] E. Getzler and R. Pandharipande, Virasoro constraints and the Chern classes of the Hodge bundle, Nucl. Phys., B530 (1998), 701-714, math.AG/9805114.

[38] L. Göttsche, Hilbert schemes of zero-dimensional subschemes of smooth varieties, Lecture Notes in Mathematics, 1572, SpringerVerlag, 1994.

[39] L. Göttsche and W. Soergel, Perverse sheaves and the cohomology of Hilbert schemes of smooth algebraic surfaces, Math. Ann., 296 (1993), 235-245.

[40] L. Göttsche and D. Zagier, Jacobi forms and the structure of Donaldson invariants for 4-manifolds with $b_{+}=1$, Selecta Math. (N.S.), 4 (1998), 69-115, alg-geom/9612020.

[41] R. Gopakumar and C. Vafa, Topological gravity as large $N$ topological gauge theory, Adv. Theor. Math. Phys., 2 (1998), 413-442, hep-th/9802016.

[42] R. Gopakumar and C. Vafa, On the gauge theory/geometry correspondence, hep-th/9811131.

[43] R. Gopakumar and C. Vafa, M-theory and topological strings-I, II, hep-th/9809187, 9812127.

[44] T. Graber and R. Pandharipande, Localization of virtual classes, Invent. Math., 135 (1999), 487-518, alg-geom/9708001.

[45] V.A. Gritsenko and V.V. Nikulin, Siegel automorphic form corrections of some Lorentzian Kac-Moody Lie algebras, Amer. J. Math., 119 (1997), 181-224, alg-geom/9504006.

[46] I. Grojnowski, Instantons and affine algebras I: the Hilbert scheme and vertex operators, Math. Res. Letters, 3 (1996), 275-291.

[47] A. Grothendieck, Techniques de construction et théorèmes d'existence en géométrie algébrique IV: Les schémas de Hilbert, Séminaire Bourbaki, 1960/61, no. 221.

[48] J. Harer and D. Zagier, The Euler characteristic of the moduli space of curves, Invent. Math., 85 (1986), 457-485. 
[49] J.A. Harvey and G. Moore, Algebras, BPS states, and strings, Nucl. Phys., B463 (1996), 315-368, hep-th/9510182.

[50] J.A. Harvey and G. Moore, On the algebras of BPS states, Commun. Math. Phys., 197 (1998), 489-519, hep-th/9609017.

[51] M. He, Espaces de modules de systèmes cohérents, Internat. J. Math., 9 (1998), 545-598.

[52] D. Hickerson, A proof of the mock theta conjectures, Invent. Math., 94 (1988), 639-660.

[53] S. Hosono, M.-H. Saito, and A. Takahashi, Holomorphic anomaly equation and BPS state counting of rational elliptic surface, Adv. Theor. Math. Phys., 3 (1999), 177-208, hep-th/9901151.

[54] B. Hunt and R. Schimmrigk, K3-fibered Calabi-Yau threefolds I, the twist map, math/9904059.

[55] D. Huybrechts, Birational symplectic manifolds and their deformations, J. Diff. Geom., 45 (1997), 488-513, alg-geom/9601015.

[56] D. Huybrechts and M. Lehn, The geometry of moduli spaces of sheaves, Vieweg, 1997.

[57] M. Jacob (ed.), Dual Theory, North-Holland, 1974.

[58] D.P. Jatkar and B. Peeters, String theory near a conifold singularity, Phys. Lett., B362 (1995), 73-77, hep-th/9508044.

[59] V.G. Kac, Infinite dimensional Lie algebras, 3rd edition, Cambridge University Press, 1990.

[60] V.G. Kac, Vertex algebras for beginners, 2nd edition, American Mathematical Society, 1998.

[61] M. Kaneko and D. Zagier, A generalized theta function and quasimodular forms, in 'The moduli space of curves', (R. Dijkgraaf, C.Faber and G. van der Geer, eds.), Progr. Math., 129, Birkhäuser, 1995.

[62] S. Katz, A. Klemm, and C. Vafa, M-theory, topological strings and spinning black holes, hep-th/9910181. 
[63] T. Kawai, $N=2$ heterotic string threshold correction, K3 surface and generalized Kac-Moody superalgebra, Phys. Lett., B372 (1996), 59-64.

[64] T. Kawai, K3 surfaces, Igusa cusp form and string theory, in 'Topological field theory, primitive forms and related topics', (M. Kashiwara, A. Matsuo, K. Saito, and I. Satake, eds.), Progr. Math., 160, Birkhäuser 1998, hep-th/9710016.

[65] T. Kawai, String duality and enumeration of curves by Jacobi forms, in 'Integrable systems and algebraic geometry', (M.-H. Saito, Y. Shimizu and K. Ueno, eds.), World Scientific 1998, hep-th/9804014.

[66] T. Kawai, Y. Yamada, and S.-K. Yang, Elliptic genera and N=2 superconformal field theory, Nucl. Phys., B414 (1994), 191-212.

[67] A. Klemm and E. Zaslow, Local mirror symmetry at higher genus, hep-th/9906046.

[68] M. Kontsevich, Homological algebra of mirror symmetry, Proceedings of the International Congress of Mathematicians, Vol. 1, 2 (Zürich, 1994), 120-139, Birkhäuser, Basel, 1995.

[69] M. Kontsevich and Yu. Manin, Gromov-Witten classes, quantum cohomology, and enumerative geometry, Commun. Math. Phys., 164 (1994), 525-562, hep-th/9402147.

[70] M. Kontsevich and Yu. Manin, Relations between the correlators of the topological sigma-model coupled to gravity, Commun. Math. Phys., 196 (1988), 385-398, alg-geom/9708024.

[71] M. Lehn, Chern classes of tautological sheaves on Hilbert schemes, math/9803091.

[72] J. Le Potier, Systèmes cohérents et structures de niveau, Astérisque, 214 (1993).

[73] J. Li and G. Tian, Virtual moduli cycles and Gromov-Witten invariants of algebraic varieties, Jour, AMS, 11 (1998), 119-174, alg-geom/9602007.

[74] M. Lübke and A. Teleman, The Kobayashi-Hitchin correspondence, World Scientific, 1995. 
[75] I.G. Macdonald, Symmetric products of an algebraic curve, Topology, 1 (1962), 319-343.

[76] Yu. Manin, Generating functions in algebraic geometry and sums over trees, in 'The moduli space of curves', (R. Dijkgraaf, C. Faber and G. van der Geer, eds.), Progr. Math., 129, Birkhäuser 1995, alg-geom/9407005.

[77] E. Markman, Brill-Noether duality for moduli spaces of sheaves on K3 surfaces, math/9901072.

[78] M. Mariño and G. Moore, Counting higher genus curves in a Calabi-Yau manifold, Nucl. Phys., B543 (1999), 592-614, hep-th/9808131.

[79] M. Mariño and G. Moore, 3-manifold topology and the DonaldsonWitten partition function, Nucl. Phys., B547 (1999), 569-598, hep-th/9811214.

[80] G. Meng and C. Taubes, $\underline{S W=M i l n o r ~ t o r s i o n, ~ M a t h . ~ R e s . ~ L e t t ., ~}$ 3 (1996), 661-674.

[81] G. Moore and E. Witten, Integration over the u-plane in Donaldson theory, Adv. Theor. Math. Phys., 1 (1997), 298-387, hep-th/9709193.

[82] J.W. Morgan and Z. Szabó, Embedded tori in four-manifolds, Topology, 38 (1999), 479-496.

[83] D.R. Morrison and C. Vafa, Compactifications of F-theory on Calabi-Yau threefolds - II, Nucl. Phys., B476 (1996), 437-469, hep-th/9603161.

[84] W. Müller, Analytic torsion and R-torsion on Riemannian manifolds, Adv. in Math., 28 (1978), 233-305.

[85] S. Mukai, Symplectic structure of the moduli space of sheaves on an abelian or K3 surface, Invent. Math., 77 (1984), 101-106.

[86] S. Mukai, On the moduli space of bundles on K3 surfaces I, in 'Vector bundles on algebraic varieties', Tata Inst. Fund. Res., 1987. 
[87] S. Mukai, Moduli of vector bundles on $K 3$ surfaces and symplectic manifolds, Sugaku Expositions, 1 (1988), 139-174.

[88] D. Mumford, Towards an enumerative geometry of the moduli space of curves, in 'Arithmetic and geometry', Vol. II, 271-328, Progr. Math., 36, Birkhäuser, 1983.

[89] H. Nakajima, Heisenberg algebra and Hilbert schemes of points on projective surfaces, Ann. Math., 145 (1997), 379-388.

[90] H. Nakajima, Lectures on Hilbert schemes of points on surfaces, American Mathematical Society, 1999.

[91] K. O'Grady, The weight-two Hodge structure of moduli spaces of sheaves on a K3 surface, J. Alg. Geom., 6 (1997), 599-644.

[92] K. O'Grady, Desingularized moduli spaces of sheaves on a K3, J. reine angew. Math., 512 (1999), 49-117.

[93] R. Pandharipande, Hodge integrals and degenerate contributions, Commun. Math. Phys., 208 (1999), 489-506, math. AG/9811140.

[94] V. Periwal, Topological closed-string interpretation of ChernSimons theory, Phys. Rev. Lett., 71 (1993), 1295-1298, hep-th/9305115.

[95] D. Ray and I. Singer, $R$-torsion and the Laplacian in Riemannian manifolds, Adv. in Math. 7 (1971) 145-210.

[96] C. Truesdell, On a function which occurs in the theory of the structure of polymers, Ann. of Math., 46 (1945), 144-157.

[97] V. Turaev, Reidemeister torsion in knot theory, Russ. Math. Surv., 41 (1986), 119-182.

[98] C. Vafa and E. Witten, A strong coupling test for S-duality, Nucl. Phys., B431 (1994), 3-77, hep-th/9408074.

[99] A. Weil, Elliptic functions according to Eisenstein and Kronecker, Ergebnisse der Mathematik und ihrer Grenzgebiete, Band 88, Springer-Verlag, 1976.

[100] K. Wirthmüller, Root systems and Jacobi forms, Compositio Math., 82 (1992), 293-354. 
[101] E. Witten, Quantum field theory and the Jones polynomial, Commun. Math. Phys., 121 (1989), 351.

[102] E. Witten, On quantum gauge theories in two dimensions, Commun. Math. Phys., 141 (1991), 153-209.

[103] E. Witten, Mirror manifolds and topological field theory, in 'Essays on mirror manifolds', (S.-T. Yau ed.), Internat. Press, 1992, hep-th/9112056.

[104] E. Witten, Chern-Simons gauge theory as a string theory, in 'The Floer memorial volume', (H. Hofer, C.H. Taubes, A. Weinstein and E. Zehnder eds.), Progr. Math., 133, Birkhäuser 1995, hep-th/9207094.

[105] E. Witten, Phases of $N=2$ theories in two dimensions, Nucl. Phys., B403 (1993), 159-222, hep-th/9301042.

[106] E. Witten, Monopoles and four-manifolds, Math. Res. Lett., 1 (1994), 769-796, hep-th/9411102.

[107] S-T. Yau and E. Zaslow, BPS states, string duality, and nodal curves on K3, Nucl. Phys., B471 (1996), 503-512, hep-th/9512121.

[108] K. Yoshioka, Some examples of Mukai's reflections on K3 surfaces, J. reine angew. Math., 515 (1999), 97-123.

[109] K. Yoshioka, Irreducibility of moduli spaces of vector bundles on K3 surfaces, math.AG/9907001.

[110] D. Zagier, The Bloch-Wigner-Ramakrishnan polylogarithm function, Math. Ann., 286 (1990), 613-624.

[111] D. Zagier, Periods of modular forms and Jacobi theta functions, Invent. Math., 104 (1991), 449-465. 